\title{
Numerical evaluation of the bispectrum in multiple field inflation
}

- the transport approach with code

\author{
Mafalda Dias ${ }^{1,2}$, Jonathan Frazer ${ }^{1,3,4}$, David J. Mulryne ${ }^{5}$ and David Seery ${ }^{2}$ \\ ${ }^{1}$ Theory Group, Deutsches Elektronen-Synchrotron, DESY, D-22603, Hamburg, Germany \\ 2 Astronomy Centre, University of Sussex, Falmer, Brighton BN1 9QH, UK \\ ${ }^{3}$ Department of Theoretical Physics, University of the Basque Country, \\ UPV/EHU 48040 Bilbao, Spain \\ ${ }^{4}$ IKerbasque, Basque Foundation for Science, 48011 Bilbao, Spain \\ ${ }^{5}$ School of Physics and Astronomy, Queen Mary University of London, Mile End Road, \\ London E1 4NS, UK \\ E-mail: mafalda.dias@desy.de, jonathan.frazer@desy.de,d.mulryne@qmul.ac.uk, \\ D.Seery@sussex.ac.uk
}

Abstract. We present a complete framework for numerical calculation of the power spectrum and bispectrum in canonical inflation with an arbitrary number of light or heavy fields. Our method includes all relevant effects at tree-level in the loop expansion, including (i) interference between growing and decaying modes near horizon exit; (ii) correlation and coupling between species near horizon exit and on superhorizon scales; (iii) contributions from mass terms; and (iv) all contributions from coupling to gravity. We track the evolution of each correlation function from the vacuum state through horizon exit and the superhorizon regime, with no need to match quantum and classical parts of the calculation; when integrated, our approach corresponds exactly with the tree-level Schwinger or 'in-in' formulation of quantum field theory. In this paper we give the equations necessary to evolve all two- and three-point correlation functions together with suitable initial conditions. The final formalism is suitable to compute the amplitude, shape, and scale dependence of the bispectrum in models with $\left|f_{\mathrm{NL}}\right|$ of order unity or less, which are a target for future galaxy surveys such as Euclid, DESI and LSST. As an illustration we apply our framework to a number of examples, obtaining quantitatively accurate predictions for their bispectra for the first time. Two accompanying reports describe publicly-available software packages that implement the method.
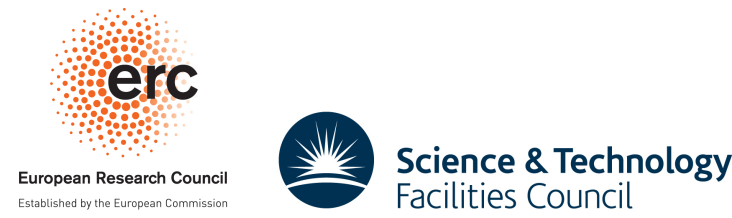


\section{Contents}

1 Introduction $\quad 2$

2 Why are automated tools necessary?

2.1 The standard calculation 5

2.2 Influence of heavy fields 8

3 Numerical computation of inflationary correlation functions at tree level 11

$\begin{array}{lll}3.1 & \text { Evolution equations } & 11\end{array}$

$\begin{array}{ll}3.2 & \text { What is included at tree level? } \\ \end{array}$

4 The tensors $u_{\mathrm{b}}^{\mathrm{a}}$ and $u^{\mathrm{a}}{ }_{\mathrm{bc}} \quad 20$

4.1 Computation of the cubic Hamiltonian 20

4.2 Equations of motion in the Heisenberg picture 23

5 The transport equations $\quad \mathbf{2 4}$

5.1 Two-point function $\quad 25$

5.2 Tensor modes 26

$\begin{array}{lll}5.3 & \text { Three-point function } & 27\end{array}$

6 Initial conditions $\quad 28$

6.1 Two-point function 28

6.2 Tensor modes 29

6.3 Three-point function 29

7 Gauge transformation to the curvature perturbation 32

8 The PyTransport and CppTransport codes $\quad 34$

9 Numerical Examples $\quad 36$

9.1 Axion-quartic model: local-mode bispectrum $\quad 37$

9.2 Single-field model with feature 41

9.3 Heavy modes: adiabatic and non-adiabatic effects 43

10 Performance and scaling behaviour $\quad 48$

10.1 Number of massless subhorizon e-folds 48

$\begin{array}{ll}10.2 \text { Shape-dependence } & 50\end{array}$

11 Conclusions $\quad 51$ 


\section{Introduction}

In the inflationary scenario, quantum-mechanical processes seeded an early distribution of gravitational potential wells. As matter sank into these wells it formed the largest structures we observe, with the result that any observable tracing this structure can be used to infer details of the seeding process. To do so we require measurements on cosmological scales, which continue to improve at a remarkable rate - and, soon, we can expect the cosmic microwave background temperature and polarization anisotropies to be joined as a precision probe by the galaxy density field, intrinsic alignments, weak lensing shear maps and perhaps others.

The raw materials for these analyses are the correlation functions describing the primordial distribution of potential wells, and to calculate them we need the methods of quantum field theory in curved spacetime. We will discuss these methods in detail below, and explain why such calculations are challenging and why analytic methods are limited. But two of the reasons are easy to state and especially difficult to overcome: first, the algebraic complexity arising when even the simplest models are coupled to gravity; and second, the occurrence of large hierarchies that compensate for the smallness of any natural expansion parameters and render naïve perturbation theory useless. The most straightforward solution to both these issues is to switch to a numerical method. Exactly this approach has been adopted in other areas of physics - including collider phenomenology, the paradigmatic example of extracting observational predictions from quantum field theory - when the same difficulties are encountered.

The tools available to assist cosmologists in these calculations are substantially less sophisticated than in collider phenomenology, where not only the numerical computations are implemented by software - it is possible to combine powerful computer packages that partially automate the calculation of LHC observables directly from a Lagrangian [1]. Examples include the Feynman diagram generators LanHEP [2,3] or FeynRules $[4,5]$ combined with CompHEP/CalcHEP [6-8], MadGraph [9] or FormCalc [10, 11]. Numerical tools for inflationary calculations have been developed, but typically they work on a case-by-case basis where derivatives of the potential must be obtained by hand and supplied as subroutines. Examples include the Fortran codes FieldInf [12-14], ModeCode and MultiModeCode [15-18], and the Python package PyFlation [19-21], which are all solvers for the two-point function in models of varying generality. For the three-point function, the only public code of which we are aware is the Fortran90 solver BINGO [22, 23] which is restricted to single-field canonical models.

Providing derivatives of the potential by hand becomes burdensome when the potential is complex or there are many fields - unfortunately, precisely the cases where numerical methods have most value. The situation is worse where a nontrivial field-space metric or kinetic structure means that further derivatives are required, such as the field-space Riemann tensor. It would be preferable, as in collider phenomenology, to automate these calculations. But automated tools have advantages beyond mere convenience, providing a fair basis for comparison between models by dropping simplifying assumptions and enabling researchers whose primary interest may be model building (rather than the calculation of correlation functions as an end in themselves) to obtain observable predictions close to the state-ofthe-art in technical sophistication. More ambitiously, once $n$-point functions are available 
there is no need to stop: we can extend the analysis to include automated calculation of late-universe observables such as the CMB angular correlation functions, the dark matter or galaxy correlation functions, estimates of scale-dependent bias, and so on.

In Ref. [24], three of us presented a Mathematica 'transport' code that can automate (in the sense just described) calculation of the inflationary two-point function in a multiplefield model with nontrivial field-space metric, given only symbolic expressions for the metric and potential. Like those described above this code is a two-point function solver. But present-day datasets already have some sensitivity to inflationary three-point functions, and this sensitivity is expected to improve as large-scale galaxy surveys such as Euclid, DESI and LSST become available. To enable model-building theorists to compare their scenarios with these datasets it would be very convenient to automate calculation of the three-point functions. In this paper we describe a numerical method for doing so - currently, applied to an arbitrary multifield model with canonical kinetic terms - and collect a number of results showcasing its utility. The method generalizes to nontrivial kinetic terms but the calculations necessary to implement it have not yet been performed. We intend to return to this in the future.

For those wishing to replicate our analyses, or apply our methods to their own models, we have made our computer codes available. The transport code described in Ref. [24] was implemented in Mathematica, which trades speed and flexibility for a certain kind of convenience inherited from access to Mathematica's symbolic engine and visualization capabilities. However, because numerical calculation of three-point functions is substantially slower than for two-point functions - and the complexity of implementation and maintenance substantially higher - it is not clear that Mathematica will continue to provide a suitable platform. To accommodate this our bispectrum codes are mostly written in compiled languages and optionally can be parallelized. They have been tested against each other but do not share code, and have different specializations and use cases:

- PyTransport is developed by a team at Queen Mary, University of London. It has a C++ core but is intended to be used through a Python interface, and uses the SymPy package to provide its symbolic algebra support. It has minimal prerequisites, supports rapid development, and its Python interface means that it is easily scriptable. It can be used with libraries such as Matplotlib or Mayavi for visualization and can be parallelized using Mpi4Py or similar packages.

- CppTransport is developed by a team at the University of Sussex. It is a pure C++ platform using the GiNaC library (originally developed as part of the one-loop particle physics project XLOOPS-GiNaC [25]) for symbolic algebra. It uses MPI multi-process communication to parallelize calculations and scales from laptop-class hardware up to many cores on a HPC cluster, without requiring a shared-memory architecture. Results are stored as SQLite databases, enabling sophisticated postprocessing using SQL database queries. For simple analyses a suite of built-in visualization and analysis tools are provided.

Both codes automate the calculation of two- and three-point field-space correlation functions (and hence two- and three-point $\zeta$ correlation functions) directly from a potential. They 
implement the same computational scheme, but in slightly different ways. In this paper our intention is to describe this scheme, illustrate its utility, and explain its relationship to earlier work. We refer to the codes when giving examples, but they will be described more fully elsewhere [26, 27]. They can be downloaded by following the links given in $\S 8$.

Synopsis.-This paper is divided into three parts. First, in $\S 2$ we review the current state-ofthe-art in computing inflationary three-point functions. This depends on accurate calculation of vacuum fluctuations over a time-dependent cosmological background for a variety of masses and parameter regimes. In some regimes it is possible to find successful analytic approximations, but in others the success of such approximations has been meagre. We argue that dramatic short-term improvements in our ability to make analytic estimates are unlikely, making a numerical method essential.

Second, in $\S \S 3-7$ we describe our numerical approach. In $\S \S 3-5$ we show that it can be regarded as a reformulation of the Schwinger or 'in-in' method to compute expectation values - as distinct from transition amplitudes, which are the output of the Feynman calculus described in particle physics textbooks. As with all formulations of field theory, ours has advantages and disadvantages. A major advantage is that it is very well suited to numerical evaluation. For example, challenging steps such as Wick rotation (that are best handled analytically) appear only in the calculation of initial conditions but not in the subsequent evolution equations. Suitable initial conditions need be calculated only once and are universal for all models, no matter what parameter regime they inhabit. We describe this calculation in $\S 6$, and the transition to $\zeta$ correlation functions in $\S 7$. The major disadvantage of our method is that it obscures the clear physical interpretation of Feynman diagrams as processes occurring in spacetime $[28,29]$. But since we intend to apply our method for numerical evaluation this is not so important.

Third, in §§8-10 we briefly describe our implementations PyTransport and CppTransport before using them to showcase the utility of the method. In $\S 9$ we verify numerically that our formalism successfully tracks the evolution of the two- and three-point functions from sub- to super-horizon scales. We demonstrate that it can be used to extract very delicate variation with shape and scale, and produce examples that exemplify some of the physical processes discussed in $§ 2$. In each case, our analysis yields results that can be obtained only using numerical techniques. In $\S 10$ we discuss the numerical characteristics of our method and its performance as implemented in PyTransport and CppTransport. In particular, we explain how the integration time and convergence properties scale with key adjustable parameters. Finally, we conclude in $§ 11$.

Notation.-We work in units where $c=\hbar=1$ and use the reduced Planck mass $M_{\mathrm{P}}^{2}=$ $(8 \pi G)^{-1}$, where $G$ is Newton's gravitational constant. Our metric signature is $(-,+,+,+)$. We work in the Heisenberg picture except where otherwise stated. In order to write concise expressions we use a number of different summation conventions. For details, see the discussion below Eq. (2.1); the discussion around Eq. (3.8); and the discussion above Eqs. (4.5a)-(4.5b). 


\section{$2 \quad$ Why are automated tools necessary?}

\subsection{The standard calculation}

Estimates of the correlation functions characterizing inflationary perturbations have been refined over several decades and are now very mature. In particular, the physical processes contributing to these correlations are clearly understood. We focus on equal-time $n$-point functions because it is these that are observationally relevant. Each correlator of this type is a function of the wavenumbers $k_{i}$ associated with its external legs, and at the time of evaluation these are typically either subhorizon in the sense $k / a H \gg 1$ or superhorizon in the sense $k / a H \ll 1$. The short transition period where $k / a H \sim 1$ is described as the epoch of horizon exit for the mode $k$.

Contributions to correlation functions.-When all wavenumbers are subhorizon, the equivalence principle means that their correlations must be nearly those of Minkowski space. ${ }^{1}$ Non-negligible correlations exist only on very short scales and are dominated by ultraviolet fluctuations. But as some wavenumbers approach horizon exit and pass to superhorizon scales it is possible for long-short correlations to develop, because the equivalence principle cannot prevent 'short' wavelength perturbations of roughly the Hubble scale from responding to the cosmological background generated by 'long' wavelength modes. At the same time, particle production processes and interference between growing and decaying modes generate a delicate pattern of correlations between the Hubble-scale modes alone. Eventually all wavenumbers move into the superhorizon regime. In this phase there are no interference effects, and the correlation functions describe an evolving ensemble of realizations of the background with different initial conditions [30-35].

An accurate calculation must capture all these effects. The difficulty of doing so varies with the model under discussion, and processes that are dominant for one class may be negligible for others. But, in general terms, there are two major challenges.

- All correlation functions receive 'quantum' contributions from a range of times around horizon exit of each momentum $k_{i}$ [36-41]. Methods to estimate these contributions are well-developed, usually yielding the result a combination of integrals over rapidlyoscillating wavefunctions [42-45]. The integrands can be interpreted as the rate per unit volume for $n$-body interactions. In some cases these integrals can be performed analytically, but in others we are already forced to rely on numerical methods. Evaluation of these rapidly oscillating 'Feynman integrals' is the first major challenge [22, 46-51].

- In models with 'adiabatic' dynamics ${ }^{2}$ the calculation is especially simple. The interaction rate per unit volume becomes negligible when all $k_{i}$ pass to superhorizon scales,

\footnotetext{
${ }^{1}$ We are assuming that the small-scale fluctuations are those of the Minkowski vacuum, described in this context as the 'Bunch-Davies vacuum'. If we allow high-energy fluctuations to have different correlation properties, perhaps as a result of nonadiabatic redshifting out of a regime controlled by exotic interactions, then more general possibilities exist.

${ }^{2}$ Here, 'adiabatic' is a term of art meaning that there is only a single independent perturabtion. This happens when the calculation is effectively single-field, in the sense that all points in spacetime follow the same inflationary trajectory through field-space.
} 
meaning that the integrals depend only on a few e-folds around horizon-exit of each wavenumber. It is only necessary to correctly estimate these contributions.

If the dynamics are not adiabatic then the situation is more complex [52]. The interaction rate per unit volume need not become negligible even on superhorizon scales, and therefore interactions may continue into the indefinite future. After subtracting effects already present in Minkowski space, it follows that each Feynman integral can receive contributions from all times when at least one wavenumber is of horizon scale or larger. (It is the unboundedly growing volume of these integrals that is responsible for spoiling naïve perturbation theory [53].) In this regime the integrand is no longer rapidly oscillatory, but because some or all wavenumbers are soft in comparison with the Hubble scale they may be exquisitely sensitive to the mass spectrum and decay channels of the model, and therefore to its microphysical details. The second major challenge is to obtain accurate estimates for all necessary masses and rates that enter this calculation, including gravitational corrections where appropriate.

Analytic approaches.-Whether we adopt analytic or numerical methods, a practical approach must overcome both these challenges. In this paper we are going to argue that numerical integration is essential for evaluation of the rate integrals, even though it introduces difficulties of its own. In particular, obtaining accurate results is nontrivial because the rapidly oscillating integrands are hard to accommodate $[22,46,47,50,51]$. We will show that this problem can be completely removed by writing 'transport' equations for each correlation function rather than attempting to directly evaluate the Feynman integrals. To overcome the second challenge we argue that automated methods based on computer algebra can be used to include all relevant terms, without the need for approximations.

In the remainder of this paper we make these arguments in more detail. But before doing so we pause to describe how the general challenges identified above manifest themselves in practical calculations. Specifically, we describe the standard tools used when making analytical estimates and identify those parts of the calculation for which automated tools offer significant benefits.

Factorization, or the 'separate universe' method.-Except for special cases, the only general method for making analytic estimates relies on factorizing each correlation function into a sum of terms. Each term contains factors capturing a contribution to one of the major effects described above. For example, we typically factorize the equal-time two-point function of the curvature perturbation $\zeta$ in the form

$$
\left\langle\zeta\left(\mathbf{k}_{1}\right) \zeta\left(\mathbf{k}_{2}\right)\right\rangle_{t}=N_{a}\left(t, t_{*}\right) N_{b}\left(t, t_{*}\right)\left\langle\delta X^{a}\left(\mathbf{k}_{1}\right) \delta X^{b}\left(\mathbf{k}_{2}\right)\right\rangle_{t_{*}} .
$$

The subscript attached to each correlation function denotes the common time of evaluation for the enclosed operators, and we assume $t>t_{*}$. The result is independent of $t_{*}$ and therefore it can be chosen to suit our convenience; normally we set $t_{*}$ to be just after the horizon-exit epoch for the common scale $k=\left|\mathbf{k}_{1}\right|=\left|\mathbf{k}_{2}\right|$. As we explain below, this makes the physical interpretation of each factor as simple as possible. 
We have collected perturbations in both the fields and their derivatives into the vector $\delta X^{a}=\left(\delta \phi^{\alpha}, \delta \dot{\phi}^{\beta}\right)$. Here, $\phi^{\alpha}$ are the fields of the model, labelled by Greek indices $\alpha, \beta, \ldots$, and $\delta \dot{\phi}^{\alpha}=\mathrm{d} \delta \phi^{\alpha} / \mathrm{d} t$. Latin indices $a, b, \ldots$ range over the combined phase space. The factorized coefficients $N_{a}\left(t, t_{*}\right)$ are nearly independent of wavenumber, and can be computed using any of a number of standard methods [33, 35, 52, 54-60].

In some circumstances the dynamics may satisfy a 'slow-roll approximation' that makes the $\dot{\phi}^{\alpha}$ functions of the field expectation values $\phi^{\alpha}$. Where this happens it is possible to collect the contribution from both field and momentum into a new coefficient $N_{\alpha}\left(t, t_{*}\right)$ and write (2.1) as a sum over fields alone. However, this simplification is not necessary for the factorization property or for our ability to make estimates.

Eq. (2.1) is the simplest example of a more general factorization principle that applies to any correlation function containing superhorizon wavenumbers [33, 53, 54]. The analogous factorization for the three-point function is

$$
\begin{aligned}
& \left\langle\zeta\left(\mathbf{k}_{1}\right) \zeta\left(\mathbf{k}_{2}\right) \zeta\left(\mathbf{k}_{3}\right)\right\rangle_{t}=N_{a} N_{b} N_{c}\left\langle\delta X^{a}\left(\mathbf{k}_{1}\right) \delta X^{b}\left(\mathbf{k}_{2}\right) \delta X^{c}\left(\mathbf{k}_{3}\right)\right\rangle_{t_{*}} \\
& +\left(N_{a b} N_{c} N_{d} \int \frac{\mathrm{d}^{3} q \mathrm{~d}^{3} r}{(2 \pi)^{3}} \delta\left(\mathbf{k}_{1}-\mathbf{q}-\mathbf{r}\right)\left\langle\delta X^{a}(\mathbf{q}) \delta X^{c}\left(\mathbf{k}_{2}\right)\right\rangle_{t_{*}}\left\langle\delta X^{b}(\mathbf{r}) \delta X^{d}\left(\mathbf{k}_{3}\right)\right\rangle_{t_{*}}\right. \\
& \quad+2 \text { permutations }) .
\end{aligned}
$$

This expression involves a new set of wavenumber-independent factorization coefficients $N_{a b}\left(t, t_{*}\right)$. To reduce clutter in Eq. (2.2) we have suppressed the time labels associated with both $N_{a}$ and $N_{a b}$. Also, we are temporarily assuming there is no large hierarchy among the $k_{i}$ so that there is a single approximate time of horizon exit $t_{*}$. For some scenarios, such as those where multiple light fields are active after horizon exit, almost everything we know about the inflationary bispectrum comes from applying factorization principles of this kind.

The utility of Eqs. (2.1) and (2.2) arises from a separation of scales. With our choice for $t_{*}$ (and continuing to assume there are no large hierarchies among the $k_{i}$ ), the 'source' or 'initial' factors $\left\langle\delta X^{a} \delta X^{b}\right\rangle_{t_{*}}$ and $\left\langle\delta X^{a} \delta X^{b} \delta X^{c}\right\rangle_{t_{*}}$ encode details of correlations between Hubble-scale modes just after horizon exit. They represent the oscillatory contribution to the Feynman rate integrals and are sensitive to particle production and interference effects around horizon exit. Meanwhile, the coefficients $N_{a}$ and $N_{a b}$ capture growing contributions associated with the superhorizon era. It is these coefficients that exhibit very strong dependence on the light part of the mass spectrum.

Analytic estimates based on factorization have been used successfully to analyse simple models. But despite this, the challenges discussed above mean that such methods do not scale efficiently to more complex cases. Expressed in the concrete language of Eqs. (2.1) and (2.2) these challenges are:

- Combinatorics. In a model with $N$ fields, estimates of $\langle\zeta \zeta\rangle$ and $\langle\zeta \zeta \zeta\rangle$ require us to compute the quantities $N_{a}, N_{a b}$ and the correlation functions $\left\langle\delta X^{a} \delta X^{b}\right\rangle,\left\langle\delta X^{a} \delta X^{b} \delta X^{c}\right\rangle$. Because of symmetries there are $2 N$ independent elements of $N_{a}$, and $2 N\left(N+\frac{1}{2}\right)$ independent elements of $N_{a b}$. Also, for fixed momenta $\mathbf{k}_{i}$ there are $2 N\left(N+\frac{1}{2}\right)$ independent elements of $\left\langle\delta X^{a} \delta X^{b}\right\rangle$ for each $k_{i}$, and $8 N^{3}$ independent elements of $\left\langle\delta X^{a} \delta X^{b} \delta X^{c}\right\rangle$ 
overall. In Table 1 we illustrate how the work required to compute $\langle\zeta \zeta\rangle$ and $\langle\zeta \zeta \zeta\rangle$-for just a single wavenumber configuration - scales with $N$.

In the most favourable cases it may be possible to use analytic approximations for $\left\langle\delta X^{a} \delta X^{b}\right\rangle$ or $\left\langle\delta X^{a} \delta X^{b} \delta X^{c}\right\rangle$, but there are already too many terms for hand calculation to be practical even at modest $N$. If a subset of these correlation functions require special treatment then the situation is much worse. The same rapidly growing number of contributions was an early catalyst for the development of automated methods in collider phenomenology.

- Estimates of Hubble-scale correlations. Eqs. (2.1) and (2.2) are useful only if the coefficients $N_{a}, N_{a b}$ and correlation functions $\left\langle\delta X^{a} \delta X^{b}\right\rangle_{t_{*}}$ and $\left\langle\delta X^{a} \delta X^{b} \delta X^{c}\right\rangle_{t_{*}}$ can be estimated separately. A number of formalisms exist to estimate $N_{a}$ and $N_{a b}$, but except in special cases the equations must be solved numerically [35, 55-60]. Therefore we are committed to some numerical effort, no matter what other choices are made.

The remaining terms are the $t_{*}$ correlators. We can estimate these analytically only in the massless limit. If all masses are light compared to the Hubble scale then this approximation is acceptable, and the estimates yield nearly 'universal' model-independent expressions depending only on the background [61]. Over the last decade, however, it has been understood that there is a rich phenomenology associated with cases where the mass spectrum extends up to the Hubble scale, or even above it. When such effects must be included the problem becomes much more complicated. Universality is almost always lost, and specialized calculations using the full machinery of quantum field theory are required.

Significant efforts have been made to produce estimates of the $t_{*}$ correlators in such models [62-71], mostly keeping only a subset of the possible effects. Nevertheless, even with such simplifications the formalism becomes cumbersome and accounting for all relevant effects is a challenge.

We briefly review the possible contributing effects in $\S 2.2$ below.

- Multiple factorization. The simple three-point function factorization formula (2.2) strictly applies only if there are no large hierarchies among the external wavenumbers $k_{i}$. Where hierarchies exist it is necessary to construct more complex factorizations-for example, using the technique of operator product expansions employed in Refs. [72-74]. The necessity for multiple factorization introduces yet further algebraic complexity.

\section{$2.2 \quad$ Influence of heavy fields}

In general, one or more of these difficulties will obstruct the use of analytic methods. This makes a numerical approach essential, whether or not we choose to adopt automated tools to mitigate algebraic complexity.

The most serious difficulties are encountered when the massless approximation does not apply, and we must obtain suitable initial conditions by some other means. This normally entails specialized estimates for the early-time correlation functions $\left\langle\delta X^{a} \delta X^{b}\right\rangle_{t_{*}}$ and 


\begin{tabular}{rrrrrrr}
\hline fields & $N_{a}$ & $N_{a b}$ & total & $\left\langle\delta X^{a} \delta X^{b}\right\rangle$ & $\left\langle\delta X^{a} \delta X^{b} \delta X^{c}\right\rangle$ & total \\
1 & 2 & 3 & 5 & 9 & 8 & 17 \\
2 & 4 & 10 & 14 & 30 & 64 & 94 \\
3 & 6 & 21 & 37 & 63 & 216 & 279 \\
4 & 8 & 36 & 44 & 108 & 512 & 620 \\
10 & 20 & 210 & 230 & 630 & 8,000 & 8,630 \\
50 & 100 & 5,050 & 5,150 & 15,150 & $1,000,000$ & $1,015,150$ \\
100 & 200 & 20,100 & 20,300 & 60,300 & $8,000,000$ & $8,060,300$ \\
\hline
\end{tabular}

Table 1. Growth in number of coefficients $N_{a}, N_{a b}$ and correlation functions $\left\langle\delta X^{a} \delta X^{b}\right\rangle$, $\left\langle\delta X^{a} \delta X^{b} \delta X^{c}\right\rangle$ that must be computed in order to estimate $\langle\zeta \zeta\rangle$ and $\langle\zeta \zeta \zeta\rangle$ using (2.1)-(2.2).

$\left\langle\delta X^{a} \delta X^{b} \delta X^{c}\right\rangle_{t_{*}}$. With this in mind we can divide multiple-field models into a number of classes:

- Massless initial conditions apply. This case occurs if all species are light relative to the Hubble scale during horizon exit of each relevant mode. There is no difficulty in estimating the initial conditions $\left\langle\delta X^{a} \delta X^{b}\right\rangle_{t_{*}}$ and $\left\langle\delta X^{a} \delta X^{b} \delta X^{c}\right\rangle_{t_{*}}$, but calculation of the coefficients $N_{a}, N_{a b}$ will normally require numerical methods. ${ }^{3}$

For models in this category the advantage of automated methods is mostly one of convenience in (effectively) computing the mass spectrum and setting up the integrals for $N_{a}$ and $N_{a b}{ }^{4}$

- Adiabatic evolution of massive fields. At the opposite extreme, massless initial conditions may fail because some modes are very heavy relative to the Hubble scale, with masses $M_{i} \gg H$. Based on experience with the decoupling theorem in Minkowski space we might expect the effect of such modes to be suppressed by inverse powers of $M_{i}$ [75].

During the last decade it has become understood that decoupling is not so trivial in a time-dependent background, because the time evolution may be associated with scales that compensate for the smallness of $1 / M_{i}$. A common example is a turn in field space associated with an angular velocity $\dot{\theta}$. If the turn is sufficiently rapid $\dot{\theta} / M_{i}$ may be order unity or larger even if $M_{i}$ is super-Hubble. However, because scales such as $\dot{\theta}$ are generated dynamically from the initial conditions they need not be visible merely from inspection of the Lagrangian.

\footnotetext{
${ }^{3}$ Factorization methods may be needed if there are hierarchies between the external wavenumbers, but the time-dependent factors appearing in these formulae are related to $N_{a}$ and $N_{a b}$, and therefore as a matter of principle the calculation contains no new elements [72-74].

${ }^{4}$ In practice, the automated tools described in this paper do not compute correlation functions by factorizing them as in Eqs. (2.1)-(2.2), and therefore do not explicitly set up integrals for $N_{a}$ and $N_{a b}$. Instead, each $n$-point function is evolved in its entirety. But if interpreted from the perspective of Eqs. (2.1)-(2.2) the outcome is the same.
} 
These $\mathrm{O}(\dot{\theta} / M)$ effects mean that care is needed when attempting to integrate-out the heavy fields. The simplest case occurs if they are heavy enough to 'adiabatically' track the minimum of their effective potential, and transverse excitations can be neglected. Even in this case, any kinetic mixing between the light and heavy fields will cause the effective potential to differ from the bare potential. This scenario was studied by Tolley \& Wyman, who demonstrated that the effective theory for the light ('gelaton') modes would be noncanonical [76]. The key feature of such models is that the speed of sound of the gelaton modes is renormalized, potentially giving a significant bispectrum amplitude on equilateral configurations $[43,44]$. The analysis was later refined by other authors [65-67, 70, 77, 78].

Automated methods offer significant advantages when applied to scenarios of this type. The first challenge is to construct either a suitable effective theory for the light modes, or to follow the evolution of the full system of light and heavy modes. Except in the very simplest scenarios it will not be possible to do either analytically, because the field trajectories cannot be expressed in closed form. Automated methods remove this difficulty by setting up the necessary computations and performing them numerically. Second, the use of an effective description involving only light modes entails an assumption that the evolution is adiabatic throughout, which need not be the case (see below). Automated calculations involving the full system of light and heavy modes automatically take nonadiabatic evolution into account.

- Nonadiabatic evolution of massive fields. Alternatively, if the time dependence of the background is sufficiently rapid then the heavy fields may evolve nonadiabatically, resulting in excitation and particle production [71, 79]. Excitation of the light fields is always important, and excitation of the heavy fields must be taken into account if they couple sufficiently strongly that energy transfer can be efficient.

Scenarios of this type were studied by Gao, Langlois \& Mizuno [70, 71]. To lowest order in the trajectory bend angle, they estimated that the corrections to the power spectrum $\mathcal{P}$ of the light mode could be written

$$
\frac{\Delta \mathcal{P}}{\mathcal{P}_{0}} \approx \mathcal{F}_{\ell}+\mathcal{F}_{h}+\mathcal{F}_{\ell h}
$$

In this language, $\mathcal{F}_{\ell}$ represents particle production in the light mode; $\mathcal{F}_{h}$ represents conversion of the heavy fluctuations into the light mode; and $\mathcal{F}_{\ell h}$ represents the response of the heavy mode to fluctuations in the light mode. The last of these is an analogue of the gelaton mechanism in which a perturbation in the light mode induces a heavy perturbation. The original gelaton behaviour described above appears in the limit where this heavy perturbation corresponds to an instantaneous adjustment into the minimum of its effective potential, preserving adiabatic evolution.

In some cases it is possible to give analytic estimates for $\mathcal{F}_{\ell}, \mathcal{F}_{h}$ and $\mathcal{F}_{\ell h}$. Ref. [70] estimated $\mathcal{F}_{\ell}$ assuming particle production to be dominantly sourced from the field zero-modes. Ref. [71] extended this to include contributions from coupling to the metric. Refs. $[70,71]$ included conversion of the vacuum wavefunction in $\mathcal{F}_{h}$ but neglected 
stimulated particle production into the heavy mode. Later, an estimate for the bispectrum induced by production of heavy particles and their subsequent decay into light modes was given in Ref. [80], at least for models with an approximate shift symmetry.

Where any of these effects are important, automated methods dramatically reduce the effort required to produce accurate calculations. The presence of particle production or strong coupling near horizon exit normally invalidates any analytic approach, meaning that $a b$ initio field theory calculations are needed to accurately estimate the initial correlation functions $\left\langle\delta X^{a} \delta X^{b}\right\rangle_{t_{*}}$ and $\left\langle\delta X^{a} \delta X^{b} \delta X^{c}\right\rangle_{t_{*}}$. Particle production into both light and heavy modes is accounted for automatically, and both memory and retardation effects are included in $\mathcal{F}_{\ell h}{ }^{5}$ (However, as we explain in $\S \S 3.2 .2-3.2 .3$, the subsequent decay of these particles into lighter species may not be captured at tree level.) Finally, if some fields remain active after horizon exit then suitable numerical integrals are needed for $N_{a}$ and $N_{a b}$ which the automated software can automatically accommodate.

- Quasi-single field inflation. The 'QSFI' scenario is a special case which can be regarded as a mix of the adiabatic and nonadiabatic cases [62,63].

We still require the evolution to be nearly adiabatic in the sense that the heavy field tracks the minimum of its effective potential, but we do not allow its mass to be much larger than $H$. This requires that the angular velocity not be too large, perhaps at most $\dot{\theta} \lesssim M \approx H$. Under these circumstances there will be negligible particle production from coupling to the background field configuration. At the same time, a Hubble-scale mass is small enough that particle production from coupling to the de Sitter metric is not completely suppressed. If we choose the cubic self-coupling to be strong, say $V^{\prime \prime \prime} \gtrsim H$, then the heavy field can acquire a sizeable bispectrum. This is communicated to the light sector by $\mathrm{O}(\dot{\theta} / M)$ quadratic mixing.

\section{Numerical computation of inflationary correlation functions at tree level}

The discussion of $\S 2.2$ makes clear that analytic approximations apply only in restricted circumstances, and must generally be complemented by numerical methods. Unfortunately, as explained in $§ 1$, numerical methods applicable to multiple-field models have so far been implemented only for the two-point function. In this paper we go further and explain how to perform numerical calculations for the three-point function. Whatever technique we employ, it should allow for arbitrary masses and couplings, capture all scale-dependent effects, and accurately track the evolution of all fluctuations both inside and outside the horizon.

\subsection{Evolution equations}

Our aim is to evaluate correlation functions of the form $\langle\mathscr{O}\rangle$, where $\mathscr{O}$ is a Heisenberg-picture operator (possibly composite) and $\langle\cdots\rangle$ denotes an expectation value defined by an 'in' state, normally chosen to match the Minkowski vacuum for subhorizon oscillators in the range of interest.

\footnotetext{
${ }^{5}$ By 'memory', we mean that $\mathcal{F}_{\ell h}$ contains an integral over time and therefore has memory of conditions at earlier times. Retardation refers to the appearance of the retarded Green's function in $\mathcal{F}_{\ell h}$.
} 
In this picture the Hamiltonian is a function $H\left(Q^{\alpha}, P_{\beta}\right)$ of the Heisenberg-picture fieldspace coordinates $Q^{\alpha}$ and their canonical momenta $P_{\beta}$. When quantized these satisfy the canonical commutation algebra $\left[Q^{\alpha}\left(\mathbf{k}_{1}, t\right), P_{\beta}\left(\mathbf{k}_{2}, t^{\prime}\right)\right]=(2 \pi)^{3} \mathrm{i} \delta_{\beta}^{\alpha} \delta\left(\mathbf{k}_{1}+\mathbf{k}_{2}\right) \delta\left(t-t^{\prime}\right)$. (In this section we are temporarily allowing the theory to be very general before specializing to the case of interest, where $Q^{\alpha}=\delta \phi^{\alpha}$.)

To define an interaction picture we divide $H$ into 'free' and 'interacting' parts,

$$
H\left(Q^{\alpha}, P_{\beta}\right)=H_{0}\left(Q^{\alpha}, P_{\beta}\right)+H_{\text {int }}\left(Q^{\alpha}, P_{\beta}\right),
$$

which at this stage remain functions of the Heisenberg-picture fields, and define new interactionpicture operators $q^{\alpha}$ and $p_{\beta}$,

$$
\begin{aligned}
& q^{\alpha}=F^{\dagger} Q^{\alpha} F \\
& p_{\beta}=F^{\dagger} P_{\beta} F,
\end{aligned}
$$

where $F$ is a unitary operator to be chosen. At least if $\mathscr{O}$ is polynomial in the $Q^{\alpha}$ and $P_{\beta}$, the correlation function $\langle\mathscr{O}\rangle$ can be written

$$
\langle\mathscr{O}\rangle=\left\langle F F^{\dagger} \mathscr{O}\left(Q^{\alpha}, P_{\beta}\right) F F^{\dagger}\right\rangle=\left\langle F \mathscr{O}\left(q^{\alpha}, p_{\beta}\right) F^{\dagger}\right\rangle .
$$

As in $\S 2.1$, we collect the operators $Q^{\alpha}, P_{\beta}$ and $q^{\alpha}, p_{\beta}$ into phase-space vectors $X^{a}=\left(Q^{\alpha}, P^{\beta}\right)$ and $x^{a}=\left(q^{\alpha}, p^{\beta}\right)$. If we choose $F$ to satisfy

$$
\frac{\mathrm{d} F}{\mathrm{~d} t}=\mathrm{i} H_{\text {int }}(X) F=\mathrm{i} F F^{\dagger} H_{\text {int }}(X) F=\mathrm{i} F H_{\text {int }}(x)
$$

then it can be verified that the equation of motion for $x^{a}$ is the Heisenberg equation for $H_{0}$,

$$
\frac{\mathrm{d} x_{a}}{\mathrm{~d} t}=-\mathrm{i}\left[x^{a}, H_{0}(x)\right] .
$$

It follows that $q^{\alpha}$ and $p_{\beta}$ can be written in terms of the wavefunctions for $H_{0}$, and the creation-annihilation operators $a_{\mathbf{k}}, a_{\mathbf{k}}^{\dagger}$ associated with the $H_{0}$ vacuum state. We denote this state by $|0\rangle$. According to a theorem of Gell-Mann \& Low, the $H_{0}$ vacuum is related to the 'in' state appearing in the inner product $\langle\mathscr{O}\rangle$ by adiabatic switch-on of the interaction $H_{\text {int }}$ in the distant past.

Eq. (3.4) and this adiabatic prescription show that the solution for $F$ is an anti-time ordered exponential when written in terms of the interaction-picture fields

$$
F=\overline{\mathrm{T}} \exp \left(\mathrm{i} \int_{-\infty^{+}}^{t} H_{\mathrm{int}}\left[x\left(t^{\prime}\right)\right] \mathrm{d} t^{\prime}\right)
$$

The symbol $\overline{\mathrm{T}}$ is the anti-time ordering operator, which rewrites its argument in order of increasing time. Its Hermitian conjugate $\mathrm{T}=\overline{\mathrm{T}}^{\dagger}$ performs the reverse operation, rewriting its argument in decreasing time order. The lower limit $-\infty^{+}$denotes that the contour of integration should be deformed above the real axis into the positive imaginary half-plane at early times, with the fields appearing in the integrand defined by analytic continuation. 
This accounts for the adiabatic switching-on required to extract the 'in'-state from the $H_{0}$ vacuum. Combining Eqs. (3.3) and (3.6) yields

$$
\langle\mathscr{O}(X)\rangle=\left\langle 0\left|\overline{\mathrm{T}} \exp \left(\mathrm{i} \int_{-\infty^{+}}^{t} H_{\mathrm{int}}\left[x\left(t^{\prime}\right)\right] \mathrm{d} t^{\prime}\right) \mathscr{O}(x) \mathrm{T} \exp \left(-\mathrm{i} \int_{-\infty^{-}}^{t} H_{\mathrm{int}}\left[x\left(t^{\prime \prime}\right)\right] \mathrm{d} t^{\prime \prime}\right)\right| 0\right\rangle .
$$

Notice that the expectation value is taken in a different state on the left- and right-hand sides. Eq. (3.7) or equivalent expressions are sometimes described as the 'in-in' or Schwinger formalism for computing expectation values [40-42].

Tree-level correlations. - The task of an automated tool is to compute (3.7) for suitable choices of operator $\mathscr{O}$. If we wish to compute up to the field-space three-point function these operators will be (returning to our original notation) $\delta X^{a} \delta X^{b}$ and $\delta X^{a} \delta X^{b} \delta X^{c}$, from which we can extract correlations functions for $\zeta \zeta$ and $\zeta \zeta \zeta$ by making a gauge transformation.

As for any interaction picture expression, the right-hand side of Eq. (3.7) admits a complex expansion into diagrams that has been discussed at length in the literature $[29,40$, $41,81,82]$. In practical calculations it is almost always necessary to truncate the expansion to a finite number of terms. Many such truncations are possible, but experience with particle physics has taught us that it is often a good approximation to restrict the expansion to a predetermined maximum number of loops. In calculations of $S$-matrix elements these loops average over quantum fluctuations that mediate the transition. For the expectation value in Eq. (3.7) there are analogous loops averaging over the effect of high energy fluctuations, but also new loops that average over intermediate particles as we describe in $§ 3.2$.

Diagrams with no loops are described as 'tree level' and contain no averaging. If nonzero, such diagrams often (but not always) constitute the leading contribution to each transition amplitude or correlation function. The precise numerical method we are going to describe computes the tree-level approximation to each correlation function, although as a matter of principle loop-level contributions could be retained at the expense of a significant increase in complexity.

Extended summation convention.- - To go further we must commit to a particular form for the Hamiltonian. When writing this and subsequent expressions it is helpful to condense our notation and include integrals over Fourier modes in the summation convention. We indicate that this extended interpretation is in use by typesetting the labels to which it applies in a sans serif face. An index contraction such as $A_{\mathrm{a}} B^{\mathrm{a}}$ therefore implies

$$
A_{\mathrm{a}} B^{\mathrm{a}}=\sum_{a} \int \frac{\mathrm{d}^{3} k_{a}}{(2 \pi)^{3}} A_{a}\left(\mathbf{k}_{a}\right) B^{a}\left(\mathbf{k}_{a}\right) .
$$

The subscript $a$ on $\mathbf{k}_{a}$ indicates that this is the Fourier mode associated with the $a$ index contraction, and because the label $a$ is set in italics the sum $\sum_{a}$ on the right-hand side involves only phase-space coordinate labels. Note that we position indices to respect the normal rules for covariant expressions, but in this paper we are always using a flat field-space metric and therefore co- or contravariant index placement has no significance.

With k-labels included there is an extra complexity because Fourier-space expressions sometimes produce the $\delta$-function $\delta_{\mathrm{ab}}=(2 \pi)^{3} \delta_{a b} \delta\left(\mathbf{k}_{a}+\mathbf{k}_{b}\right)$. When integrated out this reverses 
the sign of a k-label, which we indicate by decorating the label with a bar, as in $B^{\bar{a}}$. Hence,

$$
A_{\mathrm{a}} B^{\overline{\mathrm{a}}}=\sum_{a} \int \frac{\mathrm{d}^{3} k_{a}}{(2 \pi)^{3}} A_{a}\left(\mathbf{k}_{a}\right) B^{a}\left(-\mathbf{k}_{a}\right) .
$$

Note that contraction with $\delta^{\mathrm{a}} \mathrm{b}$ will bar an index; for example, $B^{\overline{\mathrm{a}}}=\delta^{\mathrm{ab}} B_{\mathrm{b}}$.

In this notation the canonical commutation algebra has a compact expression,

$$
\left[\delta X^{\mathrm{a}}, \delta X^{\mathrm{b}}\right]=\mathrm{i} \epsilon^{\mathrm{ab}}
$$

where $\epsilon^{\mathrm{ab}}$ is defined by

$$
\epsilon^{\mathrm{ab}}=(2 \pi)^{3} \delta\left(\mathbf{k}_{a}+\mathbf{k}_{b}\right) \epsilon^{a b}
$$

and the matrix on the right-hand side can be written in block form

$$
\epsilon^{a b}=\left(\begin{array}{cc}
0 & 1 \\
-1 & 0
\end{array}\right)
$$

In (3.12) we are assuming that the numerical values of the indices $a, b, \ldots$, are organized so that a block of field labels are followed by a block of momentum labels, in the same order. The symbol $\epsilon^{\mathrm{ab}}$ obeys the identity

$$
\epsilon^{\mathrm{ab}} \epsilon_{\mathrm{b}}{ }^{\mathrm{c}}=-\delta^{\mathrm{a} \overline{\mathrm{c}}} .
$$

The symbol $\delta^{\mathrm{ac}}$ appearing on the right-hand side behaves as a Kronecker- $\delta$ that does not bar indices; eg. $B^{\mathrm{a}}=\delta^{\mathrm{a} \overline{\mathrm{b}}} B_{\mathrm{b}}$.

Equations of motion for Heisenberg- and interaction-picture fields.-With these rules the Hamiltonian can be written

$$
H=\frac{1}{2 !} H_{\mathrm{ab}} \delta X^{\mathrm{a}} \delta X^{\mathrm{b}}+\frac{1}{3 !} H_{\mathrm{abc}} \delta X^{\mathrm{a}} \delta X^{\mathrm{b}} \delta X^{\mathrm{c}}+\cdots
$$

where '...' denotes terms of higher order in $\delta X$ that have been omitted. Without loss of generality we can assume the tensors $H_{\mathrm{ab}}$ and $H_{\mathrm{abc}}$ to be symmetric under exchange of any index pairs. In addition, each of these tensors contains a single momentum-conservation $\delta$-function, and therefore the quadratic term involves a single momentum integral, the cubic term involves two momentum integrals, and so on. The equation of motion for the Heisenberg picture fields $\delta X^{\text {a }}$ becomes

$$
\begin{aligned}
\frac{\mathrm{d} \delta X^{\mathrm{a}}}{\mathrm{d} t} & =\epsilon^{\mathrm{ac}} H_{\mathrm{cb}} \delta X^{\mathrm{b}}+\frac{1}{2 !} \epsilon^{\mathrm{ad}} H_{\mathrm{dbc}} \delta X^{\mathrm{b}} \delta X^{\mathrm{c}}+\cdots \\
& =u^{\mathrm{a}}{ }_{\mathrm{b}} \delta X^{\mathrm{b}}+\frac{1}{2 !} u^{\mathrm{a}}{ }_{\mathrm{bc}} \delta X^{\mathrm{b}} \delta X^{\mathrm{c}}+\cdots
\end{aligned}
$$

which should be regarded as a definition of the tensors $u^{\mathrm{a}} \mathrm{b}$ and $u^{\mathrm{a}} \mathrm{bc}$.

Our expression (3.7) for the correlation functions makes use of the interaction picture, and therefore depends on a division of $H$ into free and interacting parts. To make a clean statement about all tree-level graphs we should take $H_{0}$ to comprise the quadratic terms 
$H_{\mathrm{ab}} \delta X^{\mathrm{a}} \delta X^{\mathrm{b}}$, while $H_{\mathrm{int}}$ contains terms of cubic order or above. ${ }^{6}$ The equation of motion for the interaction-picture fields $\delta x^{a}$ can be written

$$
\frac{\mathrm{d} \delta x^{\mathrm{a}}}{\mathrm{d} t}=u_{\mathrm{b}}^{\mathrm{a}} \delta x^{\mathrm{b}} .
$$

Tree-level expressions for the two- and three-point functions. - We are now in a position to give formulae for the two- and three-point functions of $\delta X^{a}$ at tree-level. Eqs. (3.7) and (3.14) yield

$$
\begin{aligned}
\left\langle\delta X^{\mathrm{a}} \delta X^{\mathrm{b}}\right\rangle_{\text {tree }} & =\left\langle 0\left|\delta x^{\mathrm{a}} \delta x^{\mathrm{b}}\right| 0\right\rangle \\
\left\langle\delta X^{\mathrm{a}} \delta X^{\mathrm{b}} \delta X^{\mathrm{c}}\right\rangle_{\text {tree }} & =\left\langle 0\left|\left[\frac{\mathrm{i}}{3 !} \int^{t} H_{\text {def }} \delta x^{\mathrm{d}} \delta x^{\mathrm{e}} \delta x^{\mathrm{f}} \mathrm{d} t^{\prime}, \delta x^{\mathrm{a}} \delta x^{\mathrm{b}} \delta x^{\mathrm{c}}\right]\right| 0\right\rangle .
\end{aligned}
$$

In what follows we drop the subscript 'tree', it being understood that the $\delta X^{a}$ correlation functions are being computed only to this order. Since the state is time independent, differentiating Eq. (3.17a) and using (3.16) yields

$$
\begin{aligned}
\frac{\mathrm{d}}{\mathrm{d} t}\left\langle\delta X^{\mathrm{a}} \delta X^{\mathrm{b}}\right\rangle & =\left\langle 0\left|u^{\mathrm{a}}{ }_{\mathrm{c}} \delta x^{\mathrm{c}} \delta x^{\mathrm{b}}\right| 0\right\rangle+\left\langle 0\left|\delta x^{\mathrm{a}} u^{\mathrm{b}}{ }_{\mathrm{c}} \delta x^{\mathrm{c}}\right| 0\right\rangle \\
& =u^{\mathrm{a}}{ }_{\mathrm{c}}\left\langle\delta X^{\mathrm{c}} \delta X^{\mathrm{b}}\right\rangle+u^{\mathrm{b}}{ }_{c}\left\langle\delta X^{\mathrm{a}} \delta X^{\mathrm{c}}\right\rangle .
\end{aligned}
$$

Likewise, Eq. (3.17b) yields

$$
\begin{aligned}
\frac{\mathrm{d}}{\mathrm{d} t}\left\langle\delta X^{\mathrm{a}} \delta X^{\mathrm{b}} \delta X^{\mathrm{c}}\right\rangle= & \langle 0|\left[\frac{\mathrm{i}}{3 !} \int^{t} H_{\text {def }} \delta x^{\mathrm{d}} \delta x^{\mathrm{e}} \delta x^{\mathrm{f}} \mathrm{d} t^{\prime}, u_{\mathrm{g}}^{\mathrm{a}} \delta x^{\mathrm{g}} \delta x^{\mathrm{b}} \delta x^{\mathrm{c}}+2 \text { perms }\right]|0\rangle \\
& +\left\langle 0\left|\left[\frac{\mathrm{i}}{3 !} H_{\text {def }} \delta x^{\mathrm{d}} \delta x^{\mathrm{e}} \delta x^{\mathrm{f}}, \delta x^{\mathrm{a}} \delta x^{\mathrm{b}} \delta x^{\mathrm{c}}\right]\right| 0\right\rangle
\end{aligned}
$$

The terms in the first line produce $u^{\mathrm{a}}{ }_{\mathrm{b}}$ contracted with the three-point function in different index combinations. The second line produces

$$
\begin{aligned}
{\left[H_{\text {def }} \delta x^{\mathrm{d}} \delta x^{\mathrm{e}} \delta x^{\mathrm{f}}, \delta x^{\mathrm{a}} \delta x^{\mathrm{b}} \delta x^{\mathrm{c}}\right]=\left[H_{\text {def }} \delta x^{\mathrm{d}} \delta x^{\mathrm{e}} \delta x^{\mathrm{f}}, \delta x^{\mathrm{a}}\right] \delta x^{\mathrm{b}} \delta x^{\mathrm{c}} } \\
+\delta x^{\mathrm{a}}\left[H_{\text {def }} \delta x^{\mathrm{d}} \delta x^{\mathrm{e}} \delta x^{\mathrm{f}}, \delta x^{\mathrm{b}}\right] \delta x^{\mathrm{c}}+\delta x^{\mathrm{a}} \delta x^{\mathrm{b}}\left[H_{\text {def }} \delta x^{\mathrm{d}} \delta x^{\mathrm{e}} \delta x^{\mathrm{f}}, \delta x^{\mathrm{c}}\right],
\end{aligned}
$$

which can be rewritten

$$
\begin{aligned}
& {\left[\frac{1}{3 !} H_{\text {def }} \delta x^{\mathrm{d}} \delta x^{\mathrm{e}} \delta x^{\mathrm{f}},\right.}\left.\delta x^{\mathrm{a}} \delta x^{\mathrm{b}} \delta x^{\mathrm{c}}\right]=\frac{1}{2 !} u^{\mathrm{a}}{ }_{\mathrm{de}} \delta x^{\mathrm{d}} \delta x^{\mathrm{e}} \delta x^{\mathrm{b}} \delta x^{\mathrm{c}} \\
&+\frac{1}{2 !} u^{\mathrm{b}}{ }_{\text {de }} \delta x^{\mathrm{a}} \delta x^{\mathrm{d}} \delta x^{\mathrm{e}} \delta x^{\mathrm{c}}+\frac{1}{2 !} u^{\mathrm{c}}{ }_{\text {de }} \delta x^{\mathrm{a}} \delta x^{\mathrm{b}} \delta x^{\mathrm{d}} \delta x^{\mathrm{e}}
\end{aligned}
$$

Retaining only connected contributions gives the evolution equation

$$
\frac{\mathrm{d}}{\mathrm{d} t}\left\langle\delta X^{\mathrm{a}} \delta X^{\mathrm{b}} \delta X^{\mathrm{c}}\right\rangle=u_{\mathrm{d}}^{\mathrm{a}}\left\langle\delta X^{\mathrm{d}} \delta X^{\mathrm{b}} \delta X^{\mathrm{c}}\right\rangle+u^{\mathrm{a}}{ }_{\mathrm{de}}\left\langle\delta X^{\mathrm{d}} \delta X^{\mathrm{b}}\right\rangle\left\langle\delta X^{\mathrm{e}} \delta X^{\mathrm{c}}\right\rangle+2 \text { perms }
$$

\footnotetext{
${ }^{6}$ In analytic calculations we often choose a different division, with $H_{0}$ describing a system of massless modes and masses accommodated perturbatively in $H_{\text {int }}$. Although this division is convenient, it is useful only for fields that are light compared to the Hubble scale and is not necessary as a point of principle. Had we chosen to pursue this approach we would encounter extra complexities from the need to resum an infinite series of tree-level diagrams containing an arbitrary number of insertions of the quadratic part of $H_{\text {int }}$. To avoid this it is preferable to choose $H_{\text {int }}$ to begin at cubic order.
} 
where the permutations should respect the relative ordering of $\delta X^{\mathrm{a}}, \delta X^{\mathrm{b}}$ and $\delta X^{\mathrm{c}}$. Although we have derived these evolution equations from the interaction-picture expresssion (3.7), our final expressions are picture-independent and do not depend on the division of the Hamiltonian into $H_{0}$ and $H_{\text {int }}$. The argument given here is effectively equivalent to that given for classical superhorizon evolution in Ref. [35] and for quantum-mechanical evolution on all scales in Ref. [83], with the new element of explicitly demonstrating equivalence to the interaction-picture expression for the tree-level two- and three-point function via Eq. (3.7).

No requirement for early-time regulator.-Eqs. (3.18) and (3.22) provide an alternative means to compute the two- and three-point correlation functions. Rather than deal with the interaction-picture formula (3.7) directly, and its concomitant obligation to handle the contour deformation appearing in the time-ordered exponentials, we can instead integrate these ordinary differential equations. No contour deformations appear in these equations because each one is derived from a region where $\int_{-\infty^{ \pm}}^{t} H_{\mathrm{int}}\left[x\left(t^{\prime}\right)\right] \mathrm{d} t^{\prime}$ involves only the real $t^{\prime}$-axis. Therefore our method does not require an explicit regulator to suppress contributions to the integral on subhorizon scales. Such regulators have been widely employed in previous attempts to compute the bispectrum numerically [22, 23, 46, 47, 50, 51], but their use necessarily introduces unwanted regulator-dependence into the numerical results. Therefore care must be taken to ensure the results are regulator-independent, as far as possible; for example, see the discussion in Chen et al. [47]. By comparison our method is unaffected by such issues.

In practice, a numerical scheme based on these evolution equations requires two further elements: first, a set of suitable initial conditions for $\left\langle\delta X^{\mathrm{a}} \delta X^{\mathrm{b}}\right\rangle$ and $\left\langle\delta X^{\mathrm{a}} \delta X^{\mathrm{b}} \delta X^{\mathrm{c}}\right\rangle$; and second, algebraic expressions for $u_{\mathrm{b}}^{\mathrm{a}}$ and $u^{\mathrm{a}}{ }_{\mathrm{bc}}$. We return to these issues in $\S \S 4$ and 6 , following a short digression to understand which physical processes are included in the treelevel calculation.

\subsection{What is included at tree level?}

Our restriction to tree-level correlation functions implies that some physical effects will not be modelled perfectly. Therefore, although the tree approximation is sufficient in many scenarios, it is reasonable to ask where it might fail. In this section, without giving a detailed treatment of loop-level processes, we briefly indicate some common causes of failure. Care should be taken when using our framework (or any other tree-level scheme) to analyse the fluctuations produced in inflationary scenarios that exhibit similar phenomenology.

\subsubsection{Background evolution}

When using Feynman diagrams to compute transition amplitudes we can regard loops as averages over off-shell, virtual particles. The situation for expectation values is subtly different. Some of the loops that correct tree-level expectation values retain an interpretation as averages over virtual fluctuations, but others should be interpreted as averages over on-shell particles that are later converted to the fluctuations of interest. We will see more details of this interpretation in $\S \S 3.2 .2-3.2 .3$ below.

Loop-level terms become important whenever the averaged contribution of either effect competes with the tree-level. We will soon see that one way for this to happen is for individual 
scattering or decay processes to make important contributions to a correlation function. But there is another possibility, which is that the averaged effect of particle production modifies the inflationary trajectory by draining energy from the zero-mode. This is an example of a loop-level effect that is not captured by our tree-level formalism. Scenarios where this effect is important include 'warm' inflation [84] and trapped inflation [85]. These are both characterized by an extra friction term in the background equation of motion.

This backreaction on the zero-mode is not trivial to compute and we will not give an explicit formula for its contribution. It is not included in the in-in formula (3.7) for the correlation function $\langle\mathscr{O}\rangle$, because before deriving this formula we committed to a particular choice of background. If backreaction is important we should adjust our estimate of the background evolution, and use this adjusted trajectory in (3.7). As a matter of principle the proper adjustment can be obtained by computing the Feynman-Vernon influence functional for the back-reaction of the $k>0$ fluctuations on the $k=0$ mode [86-94]. This is a loop-level calculation.

In general, the effective equation of motion obtained from the influence functional will contain both dissipation and noise. The dissipation tracks energy lost from the zero-mode into a cloud of $k>0$ fluctuations, whereas the noise represents redeposition of energy from the cloud due to mutual annihilation of its excitations into long-wavelength modes. Neither of these effects are accounted for in our tree-level codes, although it would be straightforward to modify the background equations of motion to include an ad hoc phenomenological description. With our current technology this would have to be done on a case-by-case basis.

\subsubsection{Two-point function}

Now consider loop contributions to the two-point function (3.7). These are generated by higher-order terms from the expansion of the time-evolution operator $F$, and are present whether or not we choose to account for back-reaction into the zero-mode.

Mixing and mass effects. - The discussion in $\S 3.1$ used an exact treatment of quadratic terms in the Hamiltonian. Therefore the tree-level transport formalism already contains an exact analysis of effects from quadratic mixing or time-dependent masses, including non-adiabatic particle production. The conclusion is that our tree-level codes will correctly capture all physical effects arising from quadratic operators in the Lagrangian. For instance, this includes production of heavy particles due to a time-varying mass (including Bose enhancement), as studied recently by Flauger et al. [80]. It also includes the conversion of 'isocurvaton' fluctuations into the adiabatic mode in QSFI-like scenarios [62, 63].

Before moving on to the case of cubic- or higher-order operators, we note that in an analytic approximation we might have elected to treat time-varying quadratic terms perturbatively. This would yield a quadratic vertex as in Fig. 1. A quantitatively accurate treatment of these effects then requires that we resum all diagrams containing an arbitrary number of insertions of this vertex in any internal or external line. Although we are not following this strategy, the terms in (3.18) and (3.22) containing the two-index tensor $u_{b}^{a}$ can be regarded as performing such a resummation, by incrementally dressing each external leg of the correlation function with a copy of the mixing vertex at each time step. 


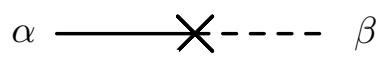

Figure 1. Quadratic mixing between species $\alpha$ and $\beta$, distinguished by the solid or dashed lines. The mixing should be regarded as a perturbative mass (possibly time dependent) in the case $\alpha=\beta$. The cross denotes insertion of a quadratic vertex.

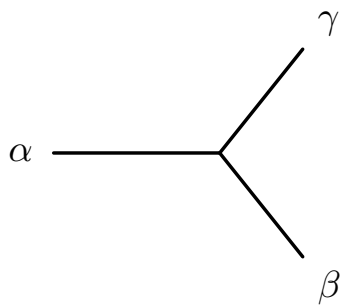

Figure 2. Tree-level S-matrix process in which a species $\alpha$ decays into a $\beta$ and $\gamma$.

Decays. - Now consider a three-body interaction described by the S-matrix process in Fig. 2. This could be regarded as the decay $\alpha \rightarrow \beta \gamma$ (or likewise for some other permutation of species), or alternatively as the reverse process $\beta \gamma \rightarrow \alpha$ in which two particles coalesce to produce a third.

A process such as Fig. 2 contributes to the two-point function $\left\langle\delta \phi^{\alpha} \delta \phi^{\beta}\right\rangle$ through a loop correction. This may be surprising, since the corresponding process occurs at tree-level in the S-matrix. To see that the conclusion is correct, insert a complete set of free-particle states $|m\rangle$ to obtain

$$
\left\langle\delta \phi^{\alpha}\left(\mathbf{k}_{1}, t\right) \delta \phi^{\beta}\left(\mathbf{k}_{2}, t\right)\right\rangle=\sum_{m}\left\langle 0\left|F(t) \delta \phi^{\alpha}\left(\mathbf{k}_{1}, t\right)\right| m\right\rangle\left\langle m\left|\delta \phi^{\beta}\left(\mathbf{k}_{2}, t\right) F^{\dagger}(t)\right| 0\right\rangle .
$$

The sum $\sum_{m}$ should be interpreted symbolically; for a continuous set of states it should be replaced by an integral with suitable measure. If we were to compute each factor appearing on the right-hand side of (3.23) by a path integral, we would arrive at the 'closed time path' representation of the in-in formalism and its characteristic doubled '+' and '-' fields [40, 89-91, 95]. The assignment of external fields between the different factors determines which correlation function is computed - eg. Wightman functions or time-ordered correlation functions. For equal-time correlation functions we need not preserve the distinction between these possibilities.

We can regard (3.23) as an average over the probability (given by the modulus-square of the corresponding S-matrix element) for the set of transitions $|0\rangle \rightarrow|m\rangle$ in which the particles in $|m\rangle$ subsequently coalesce into a $\delta \phi$ fluctuation. Each of these transitions may be individually corrected by loops, which have the same interpretation as loop corrections to an S-matrix element. When expressed as in-in diagrams they correspond to the case where all vertices have the same ' + ' or ' - ' character. Loops of this kind are genuine quantum corrections and are not included in our formalism.

In addition to these S-matrix loops, each factor in Eq. (3.23) contains contributions from pairing a subset of operators with particles in the state $|m\rangle$. The quantum numbers 


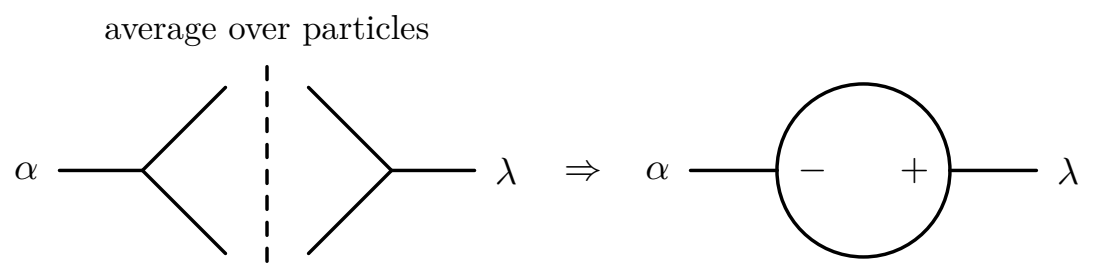

Figure 3. Loop averaging over final-state particles in Schwinger-Keldysh formalism. Left: particles in the state $|m\rangle$ are paired on either side of the 'cut', and averaged over by the sum $\sum_{m}$. Right: the same process as an in-in diagram. One loop vertex is of '-' type, and the other is of '+' type.

of the state are then averaged by the sum $\sum_{m}$. Diagrammatically, these contributions are represented by the process of Fig. 3. In the left-hand diagram we take the S-matrix element of Fig. 2 and its complex conjugate, with conjugation represented by crossing the dashed cut in the centre of the diagram. The decaying $|m\rangle$-state particles on each side of the cut are paired and averaged, yielding the loop diagram appearing on the right. ${ }^{7}$ This loop mixes ' + ' and '-' vertices. The conclusion, as advertised above, is that not all loop corrections in the Schwinger formalism represent averages over virtual particles. This is why the contribution to our expectation value from tree-level decay is nonetheless represented by a loop.

In a simple inflationary model, multiparticle production channels such as Fig. 3 are negligible in comparison with the tree-level because the dominant mechanism for particle creation is gravitational and therefore weak unless $H$ is very close to the Planck scale. ${ }^{8}$ Therefore production of two- and higher $n$-particle states is rare compared with production of a single-particle state, and the sum $\sum_{m}$ is dominated by the single-particle term. But in some models there may exist particle production mechanisms beside spontaneous creation from the vacuum. For example, this might happen if the fields roll through an enhanced symmetry point where some species become massless [85, 102-104]. In these circumstances it is possible to generate occupation numbers that are sufficiently large for multiparticle channels such as Fig. 3 to be relevant - either transiently, or in quasi-steady state if the inflationary trajectory repeatedly crosses the enhanced symmetry point. In such scenarios the tree-level prediction may be inadequate.

An explicit example is the scenario recently considered by Flauger et al. [80], in which particles of a heavy species $\chi$ are produced by rapid variation of its mass and subsequently decay into a lighter species $\phi$. As explained above, the nonadiabatic production of $\chi$ particles in their scenario is captured exactly at tree-level. But the conversion of these $\chi$ fluctuations into $\phi$ particles - and therefore their contribution to the $\phi$ correlation functions - is not captured at tree-level, precisely because it is represented by loops of the form appearing in Fig. 3.

Where multiparticle intermediate states are relevant it may be difficult to obtain a

\footnotetext{
${ }^{7}$ This interpretation is very similar to the formalism of 'cut diagrams' used to compute squared matrix elements $|\mathcal{M}|^{2}$ in hadronic physics; see eg. Refs. [96, 97].

${ }^{8}$ It can happen that the loop is enhanced by large infrared contributions [28, 40, 41, 81, 98-100], in which case multiparticle channels may become relevant if inflation is sufficiently long-lasting. Unfortunately, obtaining a robust prediction in this regime is challenging [101].
} 


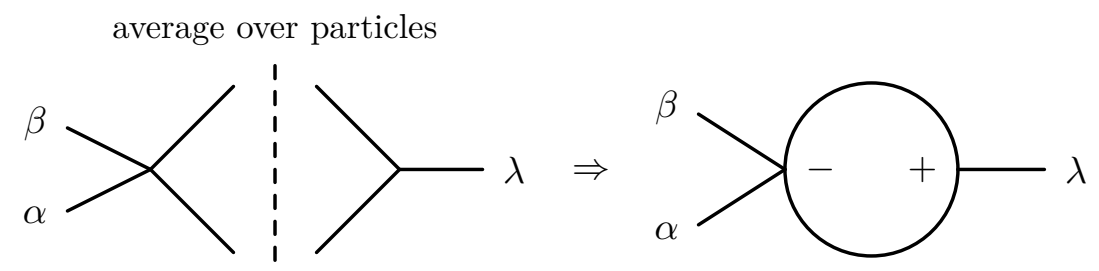

Figure 4. Loop averaging over interference between $2 \rightarrow 2$ scattering (left-hand side) and $2 \rightarrow 1$ decay (right-hand side) contributing to the inflationary bispectrum.

satisfactory description within perturbation theory. In addition to the $2 \rightarrow 1$ process of Fig. 2 there may be higher-order $n \rightarrow 1$ vertices, or repeated events, in which case further loops averaging over these transitions should be included. If many of these processes are relevant then we may be faced with failure of the loop expansion.

\subsubsection{Three-point function}

A similar discussion could be given for the three-point function (or any higher $n$-point function), although we will not do so in detail because the analysis is conceptually identical to that for the two-point function. For the three-point function, the three-body interaction of Fig. 2 is now the leading contribution. However, it can itself be corrected by $n \rightarrow 1$ or $n \rightarrow 2$ processes. One new feature is that the mixed loops may average over scattering events and not just decays; for example, consider the interference diagram of Fig. 4. This is a contribution to the three-point function from interference between $2 \rightarrow 2$ scattering and $2 \rightarrow 1$ decay.

Conclusion.--In summary, the tree-level analysis should be acceptable unless:

- quantum corrections to S-matrix elements are already important for particle scattering or decays.

- copious particle production means that multiparticle channels such as nontrivial scattering or decays contribute significantly to some $n$-point functions.

- back-reaction into the zero-mode significantly affects the inflationary trajectory.

\section{The tensors $u_{\mathrm{b}}^{\mathrm{a}}$ and $u_{\mathrm{bc}}^{\mathrm{a}}$}

In this section we return to the problem of converting the evolution (or 'transport') equations (3.18) and (3.22) into a practical numerical scheme. Our first task is to obtain explicit expressions for the tensors $u_{\mathrm{b}}^{\mathrm{a}}$ and $u_{\mathrm{b}}^{\mathrm{a}}$.

\subsection{Computation of the cubic Hamiltonian}

Background.-We are taking the matter theory to consist of a number of canonically normalized fields minimally coupled to gravity. The action is

$$
S=\frac{1}{2} \int \mathrm{d}^{4} x \sqrt{-g}\left(M_{\mathrm{P}}^{2} R-\partial_{a} \phi^{\alpha} \partial^{a} \phi_{\alpha}-2 V(\phi)\right)
$$


where indices $a, b, \ldots$ label the space-time coordinates and $V(\phi)$ is an arbitrary potential assumed to support the inflationary epoch. In this paper we mostly use a Hamiltonian formalism in which the background field equations become a first-order pair,

$$
\begin{aligned}
& \frac{\mathrm{d} \phi^{\alpha}}{\mathrm{d} t}=\pi^{\alpha} \\
& \frac{\mathrm{d} \pi^{\alpha}}{\mathrm{d} t}=-3 H \pi^{\alpha}-\delta^{\alpha \beta} V_{\beta},
\end{aligned}
$$

and $V_{\beta}=\partial_{\beta} V$ is the field-space derivative of the potential. The Hubble rate $H$ is provided by the Friedmann equation

$$
3 H^{2} M_{\mathrm{P}}^{2}=\frac{1}{2} \pi^{\alpha} \pi_{\alpha}+V .
$$

Inflation occurs whenever the slow-roll parameter $\epsilon$ is less than unity. It is defined by

$$
\epsilon \equiv-\frac{\dot{H}}{H^{2}}=\frac{\pi^{\alpha} \pi_{\alpha}}{2 M_{\mathrm{P}}^{2} H^{2}} .
$$

Perturbations.-The discussion in $\S 3.1$ shows that $u^{\mathrm{a}}{ }_{\mathrm{b}}$ and $u^{\mathrm{a}}{ }_{\mathrm{bc}}$ require knowledge of the Hamiltonian to third order in perturbations. This is a standard calculation that proceeds by writing the action as a system of coupled matter and metric fluctuations, integrating out the lapse and shift, and finally performing the Legendre transformation to the Hamiltonian. The computation was performed by Maldacena in the single field case [42] and in Ref. [61] for the more general theory (4.1). Transformation to the Hamiltonian was discussed in Ref. [81].

In this paper we do not pursue the details of this calculation but merely quote the result. We adapt the extended summation convention introduced in $\S 3.1$ for phase space labels, so that italic field-space labels $\alpha, \beta, \ldots$ range over the species of scalar fields but sans serif versions $\alpha, \beta, \ldots$ include integration over Fourier modes. The quadratic and cubic parts of the action for scalar fluctuations - including coupling to gravity but neglecting the 'tensor' spin-2 modes - can be written

$$
\begin{aligned}
& S_{2}=\frac{1}{2} \int \mathrm{d} t a^{3}\left(\delta_{\alpha \beta} \delta \dot{\phi}^{\alpha} \delta \dot{\phi}^{\beta}+M_{\alpha \beta} \delta \phi^{\alpha} \delta \phi^{\beta}\right) \\
& S_{3}=\frac{1}{2} \int \mathrm{d} t a^{3}\left(A_{\alpha \beta \gamma} \delta \phi^{\alpha} \delta \phi^{\beta} \delta \phi^{\gamma}+B_{\alpha \beta \gamma} \delta \phi^{\alpha} \delta \phi^{\beta} \delta \dot{\phi}^{\gamma}+C_{\alpha \beta \gamma} \delta \dot{\phi}^{\alpha} \delta \dot{\phi}^{\beta} \delta \phi^{\gamma}\right)
\end{aligned}
$$

The tensors $M_{\alpha \beta}, A_{\alpha \beta \gamma}, B_{\alpha \beta \gamma}$ and $C_{\alpha \beta \gamma}$ can be written in terms of corresponding coefficient tensors $M_{\alpha \beta}, A_{\alpha \beta \gamma}, B_{\alpha \beta \gamma}$ and $C_{\alpha \beta \gamma}$,

$$
\begin{aligned}
M_{\alpha \beta} & =(2 \pi)^{3} \delta\left(\mathbf{k}_{\alpha}+\mathbf{k}_{\beta}\right) M_{\alpha \beta} \\
A_{\alpha \beta \gamma} & =(2 \pi)^{3} \delta\left(\mathbf{k}_{\alpha}+\mathbf{k}_{\beta}+\mathbf{k}_{\gamma}\right) A_{\alpha \beta \gamma} \\
B_{\alpha \beta \gamma} & =(2 \pi)^{3} \delta\left(\mathbf{k}_{\alpha}+\mathbf{k}_{\beta}+\mathbf{k}_{\gamma}\right) B_{\alpha \beta \gamma} \\
C_{\alpha \beta \gamma} & =(2 \pi)^{3} \delta\left(\mathbf{k}_{\alpha}+\mathbf{k}_{\beta}+\mathbf{k}_{\gamma}\right) C_{\alpha \beta \gamma} .
\end{aligned}
$$

The $M$-tensor satisfies

$$
M_{\alpha \beta}=\frac{\mathbf{k}_{\alpha} \cdot \mathbf{k}_{\beta}}{a^{2}} \delta_{\alpha \beta}-m_{\alpha \beta}
$$


where the field-space mass-matrix $m_{\alpha \beta}$ is defined by

$$
m_{\alpha \beta}=V_{\alpha \beta}-\frac{3+\epsilon}{M_{\mathrm{P}}^{2}} \pi_{\alpha} \pi_{\beta}-\frac{\left(\pi_{\alpha} \dot{\pi}_{\beta}+\pi_{\beta} \dot{\pi}_{\alpha}\right)}{H M_{\mathrm{P}}^{2}} .
$$

Meanwhile, $A$-, $B$ - and $C$-tensors can be written

$$
\begin{aligned}
A_{\alpha \beta \gamma}= & -\frac{1}{3} V_{\alpha \beta \gamma}-\frac{\pi_{\alpha} V_{\beta \gamma}}{2 H M_{\mathrm{P}}^{2}}+\frac{\pi_{\alpha} \pi_{\beta} \xi_{\gamma}}{8 H^{2} M_{\mathrm{P}}^{4}} \\
& +\frac{\pi_{\alpha} \xi_{\beta} \xi_{\gamma}}{32 H^{3} M_{\mathrm{P}}^{4}}\left(1-\frac{\left(\mathbf{k}_{\beta} \cdot \mathbf{k}_{\gamma}\right)^{2}}{k_{\beta}^{2} k_{\gamma}^{2}}\right)+\frac{\pi_{\alpha} \pi_{\beta} \pi_{\gamma}}{8 H M_{\mathrm{P}}^{4}}\left(6-\frac{\pi_{\sigma} \pi_{\sigma}}{H^{2} M_{\mathrm{P}}^{2}}\right)+\frac{\pi_{\alpha} \delta_{\beta \gamma}}{2 H M_{\mathrm{P}}^{2}} \frac{\mathbf{k}_{\beta} \cdot \mathbf{k}_{\gamma}}{a^{2}} \\
B_{\alpha \beta \gamma}= & \frac{\pi_{\alpha} \pi_{\beta} \pi_{\gamma}}{4 H^{2} M_{\mathrm{P}}^{4}}-\frac{\pi_{\alpha} \xi_{\beta} \pi_{\gamma}}{8 H^{3} M_{\mathrm{P}}^{4}}\left(1-\frac{\left(\mathbf{k}_{\beta} \cdot \mathbf{k}_{\gamma}\right)^{2}}{k_{\beta}^{2} k_{\gamma}^{2}}\right)-\frac{\xi_{\alpha} \delta_{\beta \gamma}}{2 H M_{\mathrm{P}}^{2}} \frac{\mathbf{k}_{\alpha} \cdot \mathbf{k}_{\beta}}{k_{\alpha}^{2}} \\
C_{\alpha \beta \gamma}= & -\frac{\delta_{\alpha \beta} \pi_{\gamma}}{2 H M_{\mathrm{P}}^{2}}+\frac{\pi_{\alpha} \pi_{\beta} \pi_{\gamma}}{8 H^{3} M_{\mathrm{P}}^{4}}\left(1-\frac{\left(\mathbf{k}_{\alpha} \cdot \mathbf{k}_{\beta}\right)^{2}}{k_{\alpha}^{2} k_{\beta}^{2}}\right)+\frac{\delta_{\beta \gamma} \pi_{\alpha}}{H M_{\mathrm{P}}^{2}} \frac{\mathbf{k}_{\alpha} \cdot \mathbf{k}_{\gamma}}{k_{\alpha}^{2}} .
\end{aligned}
$$

These expressions should be symmetrized with unit weight. Specifically, $A_{\alpha \beta \gamma}$ should be symmetrized over all three indices, with corresponding exchange of $\mathbf{k}_{\alpha}, \mathbf{k}_{\beta}$ and $\mathbf{k}_{\gamma}$; and $B_{\alpha \beta \gamma}, C_{\alpha \beta \gamma}$ should be symmetrized over $\alpha \beta$, with corresponding exchange of $\mathbf{k}_{\alpha}$ and $\mathbf{k}_{\beta}$. The quantity $\xi_{\alpha}$ is defined by

$$
\xi_{\alpha}=2 \dot{\pi}_{\alpha}+\frac{\pi_{\alpha}}{H} \frac{\pi_{\beta} \pi_{\beta}}{M_{\mathrm{P}}^{2}} .
$$

Note that all these expressions are exact. In particular, they do not involve an expansion or truncation in slow-roll parameters.

Eqs. (4.7a) and (4.8a)-(4.8c) clearly demonstrate the algebraic complexity that makes precise calculations challenging in the absence of automated tools; this difficulty of accounting for all relevant microphysical details was the second major challenge identified in $\S 2.1$. In an analytic calculation it is often impractical to include all terms, and we must impose some criterion to select those that are retained. For example, in Refs. [42, 61] the slow-roll approximation was used to discard most of these contributions. In contrast, in an automated calculation based on the methods of computer algebra there is no difficulty in keeping every contribution to the Hamiltonian. This is especially important if we wish to obtain accurate predictions when the bispectrum amplitude is not large, because in these circumstances the corrections from gravitational interactions are often relevant; these are the contributions in $A_{\alpha \beta \gamma}, B_{\alpha \beta \gamma}, C_{\alpha \beta \gamma}$ and $\xi_{\alpha}$ that scale like an inverse power of $M_{\mathrm{P}}$ and therefore vanish in the decoupling limit $M_{\mathrm{P}} \uparrow \infty$. The large number of these terms makes it onerous to include them all in a manual calculation.

Transition to the Hamiltonian.-By definition, the momentum canonically conjugate to the field perturbations $\delta \phi^{\alpha}$ satisfies

$$
\delta \pi_{\alpha}(t)=\frac{\delta S}{\delta\left[\delta \dot{\phi}^{\alpha}(t)\right]},
$$

where the variational derivative is defined by

$$
\frac{\delta\left[\delta \phi^{\alpha}(t)\right]}{\delta\left[\delta \phi^{\beta}\left(t^{\prime}\right)\right]} \equiv \delta_{\beta}^{\alpha} \delta\left(t-t^{\prime}\right) .
$$


From this definition it follows that $\pi_{\beta}$ can be written

$$
\delta \pi_{\alpha}=a^{3}\left(\delta \dot{\phi}_{\alpha}+\frac{1}{2} B_{\beta \gamma \bar{\alpha}} \delta \phi^{\beta} \delta \phi^{\gamma}+C_{\bar{\alpha} \beta \gamma} \delta \dot{\phi}^{\beta} \delta \phi^{\gamma}\right) .
$$

The Hamiltonian is

$$
H=\int \mathrm{d} t\left(\delta \pi_{\alpha} \delta \dot{\phi}^{\bar{\alpha}}-L\right),
$$

considered as a functional of $\left(\delta \phi^{\alpha}, \delta \pi_{\beta}\right)$. However, to simplify (4.11) it is convenient to rescale $\delta \phi_{\alpha}$ by a factor $a^{3}, \delta \pi_{\alpha} \rightarrow a^{3} \delta \pi_{\alpha}$. This removes the overall factor of $a^{3}$ from (4.11) and means that each term in the Hamiltonian has the same common factor appearing in the measure. In terms of the rescaled momentum we find that the time derivative of the field perturbation can be written

$$
\delta \dot{\phi}_{\alpha}=\delta \pi_{\alpha}-\frac{1}{2} B_{\beta \gamma \bar{\alpha}} \delta \phi^{\beta} \delta \phi^{\gamma}-C_{\bar{\alpha} \beta \gamma} \delta \pi^{\beta} \delta \phi^{\gamma}+\cdots,
$$

where '.. ' denotes higher-order interactions that do not need to be retained at the order to which we are working. Rearranging $H$ and neglecting further higher-order interactions yields

$$
\begin{aligned}
H=\frac{1}{2} \int \mathrm{d} t a^{3}( & \delta_{\alpha \beta} \delta \pi^{\alpha} \delta \pi^{\beta}-M_{\alpha \beta} \delta \phi^{\alpha} \delta \phi^{\beta} \\
& \left.-A_{\alpha \beta \gamma} \delta \phi^{\alpha} \delta \phi^{\beta} \delta \phi^{\gamma}-B_{\alpha \beta \gamma} \delta \phi^{\alpha} \delta \phi^{\beta} \delta \pi^{\gamma}-C_{\alpha \beta \gamma} \delta \pi^{\alpha} \delta \pi^{\beta} \delta \phi^{\gamma}\right)
\end{aligned}
$$

There are no barred indices in this expression. The first two terms contribute to the quadratic piece $H_{\mathrm{ab}} \delta X^{\mathrm{a}} \delta X^{\mathrm{b}}$ and therefore $H_{0}$, while the last three terms contribute to the cubic piece $H_{\mathrm{abc}} \delta X^{\mathrm{a}} \delta X^{\mathrm{b}} \delta X^{\mathrm{c}}$ and therefore constitute $H_{\text {int }}$. In this example $H_{\text {int }}$ has the appearance of $-L_{\text {int }}$ with $\delta \dot{\phi}^{\alpha} \rightarrow \delta \pi^{\alpha}$, but it should be remembered that $\delta \pi^{\alpha} \neq \delta \dot{\phi}^{\alpha}$ once interactions are included. ${ }^{9}$ This will be important in the subsequent discussion.

\subsection{Equations of motion in the Heisenberg picture}

Given the Hamiltonian (4.14) we can extract the $u$-tensors $u^{\mathrm{a}}{ }_{\mathrm{b}}$ and $u^{\mathrm{a}}{ }_{\mathrm{bc}}$ by comparison with the Heisenberg equations (3.15). These assume a slightly noncanonical form when using our rescaled momentum $\delta \pi_{\alpha}$,

$$
\begin{aligned}
& \frac{\mathrm{d} \delta \phi^{\alpha}}{\mathrm{d} t}=-\mathrm{i}\left[\delta \phi^{\alpha}, H\right] \\
& \frac{\mathrm{d} \delta \pi_{\alpha}}{\mathrm{d} t}=-\mathrm{i}\left[\delta \pi_{\alpha}, H\right]-3 H \delta \pi_{\alpha} .
\end{aligned}
$$

Also, the operators $\delta \phi^{\alpha}$ and $\delta \pi_{\alpha}$ acquire a modified commutation algebra,

$$
\left[\delta \phi^{\alpha}(t), \delta \pi_{\beta}\left(t^{\prime}\right)\right]=\mathrm{i} a^{3} \delta_{\beta}^{\alpha} \delta\left(t-t^{\prime}\right)
$$

The noncanonical term $-3 H \delta \pi_{\alpha}$ appearing in the $\delta \pi_{\alpha}$ evolution equation can be absorbed as an extra contribution to $u^{\mathrm{a}}{ }_{\mathrm{b}}$.

\footnotetext{
${ }^{9} \mathrm{Had}$ our action $(4.5 \mathrm{a})-(4.5 \mathrm{~b})$ contained terms cubic in $\delta \dot{\phi}$ then even the symbolic appearance $H_{\mathrm{int}}=-L_{\mathrm{int}}$ would not have been maintained.
} 
To proceed we could use Eqs. (4.15a)-(4.15b) and Eq. (4.16) in conjunction with (4.14), or just read off $H_{\mathrm{ab}}$ and $H_{\mathrm{abc}}$ from (4.14) and directly compute $u_{\mathrm{b}}^{\mathrm{a}}=\epsilon^{\mathrm{ac}} H_{\mathrm{cb}}$ and $u_{\mathrm{bc}}^{\mathrm{a}}=$ $\epsilon^{\mathrm{ad}} H_{\mathrm{dbc}}$. Following the latter route we see that $H_{\mathrm{ab}}$ can be written

$$
H_{\mathrm{ab}}=\left(\begin{array}{cc}
-M_{\alpha \beta} & 0 \\
0 & \delta_{\alpha \beta}
\end{array}\right),
$$

where we are assuming the same numerical arrangement for the indices a, b used in Eq. (3.12), and $\alpha, \beta$ are the field-space indices corresponding to the phase-space indices $a, b$. (Explicitly, $\alpha=a \bmod N, \beta=b \bmod N$ where $N$ is the number of fields.) It follows that $u^{\text {a }}{ }_{\mathrm{b}}$ satisfies

$$
u_{\mathrm{b}}^{\mathrm{a}}=\left(\begin{array}{cc}
0 & 1 \\
-1 & 0
\end{array}\right)\left(\begin{array}{cc}
-M_{\beta}^{\bar{\alpha}} & 0 \\
0 & \delta_{\beta}^{\bar{\alpha}}
\end{array}\right)+\left(\begin{array}{cc}
0 & 0 \\
0 & -3 H \delta_{\beta}^{\bar{\alpha}}
\end{array}\right)=\left(\begin{array}{cc}
0 & \delta_{\beta}^{\bar{\alpha}} \\
M_{\beta}^{\bar{\alpha}}-3 H \delta_{\beta}^{\bar{\alpha}}
\end{array}\right) .
$$

Proceeding to the cubic terms, we find that $H_{\mathrm{abc}}$ satisfies

$$
H_{\mathrm{abc}}=\left\{\begin{array}{c}
\left(\begin{array}{cc}
-3 A_{\alpha \beta \gamma} & -B_{\alpha \gamma \beta} \\
-B_{\beta \gamma \alpha} & -C_{\alpha \beta \gamma}
\end{array}\right) \\
\left(\begin{array}{cc}
-B_{\alpha \beta \gamma} & -C_{\gamma \beta \alpha} \\
-C_{\alpha \gamma \beta} & 0
\end{array}\right)
\end{array}\right\}
$$

In this formula, the a index labels rows on each $2 \times 2$ block matrix; the $\mathrm{b}$ index labels corresponding columns; and the $c$ index labels the matrices within the braces $\{\cdots\}$. As above, the indices $\alpha, \beta \gamma$ are the field-space versions of $a, b, c$. Likewise, $\mathbf{k}_{\alpha}$ should be regarded as equal to $\mathbf{k}_{a}, \mathbf{k}_{\beta}$ should be regarded as equal to $\mathbf{k}_{b}$, and so on.

A short calculation yields $u^{\mathrm{a}} \mathrm{bc}$,

$$
u_{\mathrm{bc}}^{\mathrm{a}}=\left\{\begin{array}{c}
\left(\begin{array}{cc}
0 & \mathbf{1} \\
-1 & 0
\end{array}\right)\left(\begin{array}{cc}
-3 A^{\bar{\alpha}}{ }_{\beta \gamma} & -B^{\bar{\alpha}}{ }_{\gamma \beta} \\
-B_{\beta \gamma}{ }^{\bar{\alpha}} & -C^{\bar{\alpha}_{\beta \gamma}}
\end{array}\right) \\
\left(\begin{array}{cc}
0 & 1 \\
-1 & 0
\end{array}\right)\left(\begin{array}{cc}
-B_{\beta \gamma}^{\bar{\alpha}} & -C_{\gamma \beta}^{\bar{\alpha}} \\
-C_{\gamma \beta}^{\bar{\alpha}} & 0
\end{array}\right)
\end{array}\right\}=\left\{\begin{array}{c}
\left(\begin{array}{cc}
-B_{\beta \gamma}^{\bar{\alpha}} & -C^{\bar{\alpha}}{ }_{\beta \gamma} \\
3 A_{\beta \gamma}^{\bar{\alpha}} & B_{\gamma \beta}^{\bar{\alpha}_{\gamma \beta}}
\end{array}\right) \\
\left(\begin{array}{cc}
-C_{\gamma \beta}^{\bar{\alpha}} & 0 \\
B_{\beta \gamma}^{\bar{\alpha}_{\beta \gamma}} & C_{\gamma \beta}{ }^{\bar{\alpha}}
\end{array}\right)
\end{array}\right\}
$$

\section{The transport equations}

We now have all the elements in place to write an evolution equation for each configuration of the two- and three-point functions. First, notice that the arrangement of barred indices in Eqs. (4.18) and (4.20) implies that we can define coefficient tensors $u_{b}^{a}$ and $u_{b c}^{a}$ that satisfy

$$
\begin{aligned}
u_{\mathrm{b}}^{\mathrm{a}} & =(2 \pi)^{3} \delta\left(\mathbf{k}_{a}-\mathbf{k}_{b}\right) u_{b}^{a}\left(\mathbf{k}_{a}, \mathbf{k}_{b}\right) \\
u_{\mathrm{bc}}^{\mathrm{a}} & =(2 \pi)^{3} \delta\left(\mathbf{k}_{a}-\mathbf{k}_{b}-\mathbf{k}_{c}\right) u_{b c}^{a}\left(\mathbf{k}_{a}, \mathbf{k}_{b}, \mathbf{k}_{c}\right) .
\end{aligned}
$$

In these definitions we should still regard $\mathbf{k}_{a}$ as the momentum associated with the index $a, \mathbf{k}_{b}$ as the momentum associated with $b$, et cetera, but we have written this dependence explicitly because our convention of labelling momenta by the corresponding index will break 
down when we come to write an evolution equation for a single wavenumber configuration in Eq. (5.14) below.

The coefficient tensors $u_{b}^{a}$ and $u_{b c}^{a}$ are given by expressions obtained symbolically from Eqs. (4.18) and (4.20), by replacing extended summation-convention indices $(\alpha, \beta, \ldots)$ with standard indices $(\alpha, \beta, \ldots)$. Note that because the coefficient tensors $M_{\alpha \beta}, A_{\alpha \beta \gamma}, B_{\alpha \beta \gamma}$ and $C_{\alpha \beta \gamma}$ depend on the momenta, it is still necessary to keep track of barred labels indicating that the corresponding sign should be reversed. However, bars on momenta-independent tensors such as $\delta_{\beta}^{\alpha}$ can be safely discarded.

There is a further simplification that can be made. In principle, $u_{b}^{a}$ is a function of the momenta $\mathbf{k}_{a}$ and $\mathbf{k}_{b}$. However, because the $\delta$-function in (5.1a) constrains these to be equal we can replace it with the equivalent form

$$
\tilde{u}_{b}^{a}(k)=\left(\begin{array}{cc}
0 & \delta_{\beta}^{\alpha} \\
\tilde{M}^{\alpha}{ }_{\beta} & -3 H \delta_{\beta}^{\alpha}
\end{array}\right)
$$

and $\tilde{M}_{\alpha \beta}$ is a rewriting of $(4.7 \mathrm{a})$,

$$
\tilde{M}_{\alpha \beta}=-\frac{k^{2}}{a^{2}} \delta_{\alpha \beta}-m_{\alpha \beta} .
$$

This accounts for sign reversal from the barred index $\bar{\alpha}$ and uses the common magnitude $k$ of $\mathbf{k}_{a}$ and $\mathbf{k}_{b}$.

\subsection{Two-point function}

It is now straightforward to extract an evolution equation for the two-point function. The fully momentum-dependent transport equation is Eq. (3.18). To convert this to an ordinary differential equation we appeal to statistical isotropy and homogeneity and write the equaltime two-point function in the form

$$
\left\langle\delta X^{\mathrm{a}} \delta X^{\mathrm{b}}\right\rangle=(2 \pi)^{3} \delta\left(\mathbf{k}_{a}+\mathbf{k}_{b}\right) \Sigma^{a b}(k),
$$

where as above $k=\left|\mathbf{k}_{a}\right|=\left|\mathbf{k}_{b}\right|$ is the common magnitude of the momenta. Then Eqs. (3.18), (5.1b), (5.2) and (5.4) combine to give

$$
\frac{\mathrm{d} \Sigma^{a b}(k)}{\mathrm{d} t}=\tilde{u}_{c}^{a}(k) \Sigma^{c b}(k)+\tilde{u}_{c}^{b}(k) \Sigma^{a c}(k) .
$$

Reality and symmetry properties. - The two-point function $\Sigma^{a b}(k)$ will generally be complex, and a practical numerical implementation must evolve its real and imaginary parts separately. Fortunately the linearity of Eq. (5.5) makes this straightforward. We break $\Sigma^{a b}$ into real and imaginary parts

$$
\Sigma^{a b}=\Sigma_{\mathrm{Re}}^{a b}+\mathrm{i} \Sigma_{\mathrm{Im}}^{a b},
$$

where both $\Sigma_{\mathrm{Re}}$ and $\Sigma_{\operatorname{Im}}$ are real. Then, because $\tilde{u}_{b}^{a}$ is real, each of $\Sigma_{\mathrm{Re}}$ and $\Sigma_{\operatorname{Im}}$ obey Eq. (5.5) separately. We only require the real part to compute the $\zeta$ two-point function $\langle\zeta \zeta\rangle$, but we will see below that computation of $\left\langle\delta X^{\mathrm{a}} \delta X^{\mathrm{b}} \delta X^{\mathrm{c}}\right\rangle$ or $\langle\zeta \zeta \zeta\rangle$ requires knowledge of both the real and imaginary parts. 
It follows from the relation $\langle\mathscr{O}\rangle^{*}=\left\langle\mathscr{O}^{\dagger}\right\rangle$ (where $*$ denotes conjugation of a complex number, and $\dagger$ denotes Hermitian conjugation of an operator) that $\Sigma_{\text {Re }}$ is symmetric and $\Sigma_{\operatorname{Im}}$ is antisymmetric,

$$
\begin{aligned}
& \Sigma_{\mathrm{Re}}^{a b}=\Sigma_{\mathrm{Re}}^{b a} \\
& \Sigma_{\mathrm{Im}}^{a b}=-\Sigma_{\mathrm{Im}}^{b a} .
\end{aligned}
$$

The imaginary part normally decays outside the horizon.

\subsection{Tensor modes}

We can compute a transport equation for the components of the tensor 2-point function in a similar way; for details see Ref. [24]. Here we summarize the calculation.

Tensor perturbations $\gamma$ are transverse, traceless perturbations of the spatial metric. They represent gravitational waves excited by the inflationary epoch. Up to quadratic order their evolution is controlled by the action

$$
S \supseteq \frac{M_{\mathrm{P}}^{2}}{8} \int \mathrm{d}^{3} x \mathrm{~d} t a^{3}\left\{\dot{\gamma}_{i j} \dot{\gamma}^{i j}-\partial_{k} \gamma_{i j} \partial^{k} \gamma^{i j}\right\}
$$

The indices $i, j, k, \ldots$ label spatial coordinates. To write a scalar transport equation it is convenient to decompose $\boldsymbol{\gamma}$ into a basis of polarization tensors. Working in Fourier space, we write

$$
\gamma(\mathbf{x})=\int \frac{\mathrm{d}^{3} k}{(2 \pi)^{3}} \sum_{s} \gamma_{s}(\mathbf{k}) \boldsymbol{e}^{s}(\mathbf{k}) \mathrm{e}^{i \mathbf{k} \cdot \mathbf{x}}
$$

where $s=\{+, \times\}$ labels the available polarization states, and the polarization tensors $\boldsymbol{e}(\mathbf{k})$ satisfy the relations $\mathbf{k} \cdot \boldsymbol{e}^{s}(\mathbf{k})=0$ and $\operatorname{tr}\left(\boldsymbol{e}^{s} \cdot \boldsymbol{e}^{s^{\prime}}\right)=2 \delta^{s s^{\prime}}$. Each polarization $M_{\mathrm{P}} \gamma_{s}(\mathbf{k}) / \sqrt{2}$ behaves as a canonically-normalized massless scalar field.

In analogy with the case of field-space fluctuations we define a tensor momentum $\pi_{s}=$ $\mathrm{d} \gamma_{s} / \mathrm{d} t$ and collect $\gamma_{s}$ and $\pi_{s}$ into a two-component vector $Y_{s}^{\mathfrak{a}}=\left(\gamma_{s}, \pi_{s}\right)$. Gothic indices $\mathfrak{a}, \mathfrak{b}$, ... label the components of this vector. The two-point function can be written

$$
\left\langle Y_{s}^{\mathfrak{a}}\left(\mathbf{k}_{1}\right) Y_{s^{\prime}}^{\mathfrak{b}}\left(\mathbf{k}_{2}\right)\right\rangle=(2 \pi)^{3} \delta_{s s^{\prime}} \delta\left(\mathbf{k}+\mathbf{k}^{\prime}\right) \Gamma^{\mathfrak{a} \mathfrak{b}}(k)
$$

Then it follows from the same argument leading to (5.5) that the transport equation for $\Gamma^{\mathfrak{a} \mathfrak{b}}$ can be written

$$
\frac{\mathrm{d} \Gamma^{\mathfrak{a} \mathfrak{b}}(k)}{\mathrm{d} t}=w^{\mathfrak{a}}{ }_{\mathfrak{c}}(k) \Gamma^{\mathfrak{c} \mathfrak{b}}(k)+w^{\mathfrak{b}}{ }_{\mathfrak{c}}(k) \Gamma^{\mathfrak{a} \mathfrak{c}}(k)+\cdots .
$$

The functional form of the matrices $w^{\mathfrak{a}} \mathfrak{b}$ follows directly from the massless limit of Eqs. (5.2) and (5.3), that is,

$$
w_{\mathfrak{b}}^{\mathfrak{a}}(k)=\left(\begin{array}{cc}
0 & 1 \\
-k^{2} / a^{2} & -3 H
\end{array}\right)
$$

The coefficient matrix $\Gamma^{\mathfrak{a} \mathfrak{b}}$ will generally be complex on subhorizon scales, as in $\S 5.1$. For the same reasons explained above the real and imaginary components evolve independently at tree-level. The imaginary part decays outside the horizon and does not affect observables. Therefore for practical purposes we can restrict our attention to the real part. 


\subsection{Three-point function}

Now consider the three-point function. Statistical isotropy and homogeneity imply that this takes the form

$$
\left\langle\delta X^{\mathrm{a}} \delta X^{\mathrm{b}} \delta X^{\mathrm{c}}\right\rangle=(2 \pi)^{3} \delta\left(\mathbf{k}_{a}+\mathbf{k}_{b}+\mathbf{k}_{c}\right) B^{a b c}\left(k_{a}, k_{b}, k_{c}\right) .
$$

The momentum-dependent transport equation is now (3.22). Applied to Eq. (5.13) we find

$$
\begin{aligned}
\frac{\mathrm{d} B^{a b c}\left(k_{a}, k_{b}, k_{c}\right)}{\mathrm{d} t}= & \tilde{u}_{d}^{a}\left(k_{a}\right) B^{d b c}\left(k_{a}, k_{b}, k_{c}\right)+\tilde{u}_{d}^{b}\left(k_{b}\right) B^{a d c}\left(k_{a}, k_{b}, k_{c}\right)+\tilde{u}_{d}^{c}\left(k_{c}\right) B^{a b d}\left(k_{a}, k_{b}, k_{c}\right) \\
& +u_{d e}^{a}\left(\mathbf{k}_{a},-\mathbf{k}_{b},-\mathbf{k}_{c}\right) \Sigma^{d b}\left(k_{b}\right) \Sigma^{e c}\left(k_{c}\right) \\
& +u_{d e}^{b}\left(\mathbf{k}_{b},-\mathbf{k}_{a},-\mathbf{k}_{c}\right) \Sigma^{a d}\left(k_{a}\right) \Sigma^{e c}\left(k_{c}\right) \\
& +u_{d e}^{c}\left(\mathbf{k}_{c},-\mathbf{k}_{a},-\mathbf{k}_{b}\right) \Sigma^{a d}\left(k_{a}\right) \Sigma^{b e}\left(k_{c}\right) .
\end{aligned}
$$

We have written out all terms explicitly in order to avoid ambiguity about the index ordering. This is important in the $\Sigma \Sigma$ terms because, as explained above, $\Sigma^{a b}$ is not symmetric if it contains a significant imaginary part.

Comparison with Eq. (4.20) shows that the pattern of sign reversals in these terms corresponds to reversal of all momenta in Eqs. (4.8a)-(4.8c). However, because the momenta appear only in inner products with each other (and therefore always occur in pairs), this is the same as reversing none of them. Also, like the two-point function, $B^{a b c}$ will normally be complex and should be broken into its real and imaginary parts,

$$
B^{a b c}\left(k_{1}, k_{2}, k_{3}\right)=B_{\operatorname{Re}}^{a b c}\left(k_{1}, k_{2}, k_{3}\right)+\mathrm{i} B_{\operatorname{Im}}^{a b c}\left(k_{1}, k_{2}, k_{3}\right) .
$$

We will see below that the imaginary part is zero at tree-level and therefore plays no role in the calculation. Putting all this together, the ordinary differential equation we are seeking for $B_{\mathrm{Re}}^{a b c}$ can be written

$$
\begin{aligned}
\frac{\mathrm{d} B_{\operatorname{Re}}^{a b c}\left(k_{a}, k_{b}, k_{c}\right)}{\mathrm{d} t}= & \tilde{u}_{d}^{a}\left(k_{a}\right) B_{\operatorname{Re}}^{d b c}\left(k_{a}, k_{b}, k_{c}\right)+\tilde{u}_{d}^{b}\left(k_{b}\right) B_{\operatorname{Re}}^{a d c}\left(k_{a}, k_{b}, k_{c}\right)+\tilde{u}_{d}^{c}\left(k_{c}\right) B_{\operatorname{Re}}^{a b d}\left(k_{a}, k_{b}, k_{c}\right) \\
& +\tilde{u}_{d e}^{a}\left(\mathbf{k}_{a}, \mathbf{k}_{b}, \mathbf{k}_{c}\right) \Sigma_{\operatorname{Re}}^{d b}\left(k_{b}\right) \Sigma_{\operatorname{Re}}^{e c}\left(k_{c}\right)-\tilde{u}_{d e}^{a}\left(\mathbf{k}_{a}, \mathbf{k}_{b}, \mathbf{k}_{c}\right) \Sigma_{\operatorname{Im}}^{d b}\left(k_{b}\right) \Sigma_{\operatorname{Im}}^{e c}\left(k_{c}\right) \\
& +\tilde{u}_{d e}^{b}\left(\mathbf{k}_{b}, \mathbf{k}_{a}, \mathbf{k}_{c}\right) \Sigma_{\operatorname{Re}}^{a d}\left(k_{a}\right) \Sigma_{\operatorname{Re}}^{e c}\left(k_{c}\right)-\tilde{u}_{d e}^{b}\left(\mathbf{k}_{b}, \mathbf{k}_{a}, \mathbf{k}_{c}\right) \Sigma_{\operatorname{Im}}^{a d}\left(k_{a}\right) \Sigma_{\operatorname{Im}}^{e c}\left(k_{c}\right) \\
& +\tilde{u}_{d e}^{c}\left(\mathbf{k}_{c}, \mathbf{k}_{a}, \mathbf{k}_{b}\right) \Sigma_{\operatorname{Re}}^{a d}\left(k_{a}\right) \Sigma_{\operatorname{Re}}^{b e}\left(k_{b}\right),-\tilde{u}_{d e}^{c}\left(\mathbf{k}_{c}, \mathbf{k}_{a}, \mathbf{k}_{b}\right) \Sigma_{\operatorname{Im}}^{a d}\left(k_{a}\right) \Sigma_{\operatorname{Im}}^{b e}\left(k_{b}\right),
\end{aligned}
$$

where $\tilde{u}_{b c}^{a}$ is defined by the plain summation-convention version of Eq. (4.20) but with sign reversal discarded,

$$
\tilde{u}_{b c}^{a}=\left\{\begin{array}{c}
\left(\begin{array}{cc}
-B_{\beta \gamma}{ }^{\alpha} & -C^{\alpha}{ }_{\beta \gamma} \\
3 A^{\alpha}{ }_{\beta \gamma} & B^{\alpha}{ }_{\gamma \beta}
\end{array}\right) \\
\left(\begin{array}{cc}
-C^{\alpha}{ }_{\gamma \beta} & 0 \\
B^{\alpha}{ }_{\beta \gamma} & C_{\gamma \beta}{ }^{\alpha}
\end{array}\right)
\end{array}\right\} .
$$

We have not written the $\mathbf{k}$ dependence explicitly, but the left-hand side should be regarded as a function of the momenta $\mathbf{k}_{a}, \mathbf{k}_{b}, \mathbf{k}_{c}$ associated with each index. On the right-hand side, the tensors $A_{\alpha \beta \gamma}, B_{\alpha \beta \gamma}$ and $C_{\alpha \beta \gamma}$ are functions of momenta $\mathbf{k}_{\alpha}, \mathbf{k}_{\beta}, \mathbf{k}_{\gamma}$ that should be obtained from the assignments $\mathbf{k}_{a} \rightarrow \mathbf{k}_{\alpha}, \mathbf{k}_{b} \rightarrow \mathbf{k}_{\beta}, \mathbf{k}_{c} \rightarrow \mathbf{k}_{\gamma}$. 


\section{Initial conditions}

Finally, we must provide initial conditions for $\Sigma_{\mathrm{Re}}^{a b}, \Sigma_{\mathrm{Im}}^{a b}$ and $B_{\mathrm{Re}}^{a b c}$. In our framework these are computed analytically from the interaction-picture expression (3.7).

This proposition might appear hopeless because the premise of our argument is that analytic approximations to Eq. (3.7) are either prohibitively complex or cannot capture the relevant physical processes such as particle creation and energy exchange. However, as we now explain, it is normally possible to use (3.7) to obtain accurate initial conditions provided the initial time is taken sufficiently early that the relevant modes are deep in the subhorizon era.

Inspection of Eq. (4.7a) shows that the mass-matrix $m_{\alpha \beta}$ defined in (4.7b) models the influence of complex processes such as energy exchange in the two-point function. The three-point function inherits all of these effects, and also includes others from the threebody vertices (4.8a)-(4.8c). But at very early times the term $(k / a)^{2}$ in $M_{\alpha \beta}$ will dominate $m_{\alpha \beta}$ if all eigenvalues of the mass matrix remain bounded. Therefore, at sufficiently early times, the two-point function will approach that for a set of massless uncoupled scalar fields. This is much easier to handle analytically. Meanwhile, for the three-point function, the kdependent terms in Eqs. (4.8a)-(4.8c) will dominate. These significant simplifications mean that analytic approximations become both tractable and accurate.

\subsection{Two-point function}

We define $m^{2}$ to be the largest eigenvalue of the mass matrix (4.7b). Provided this eigenvalue remains bounded the kinetic term $(k / a)^{2}$ becomes increasingly important at early times, while contributions from the mass matrix become irrelevant. If we choose the initial time so that $(k / a)^{2} \gg m^{2}$ then the fields can be approximated as massless and non-interacting with very good accuracy. The corresponding time-ordered unequal-time two-point function in a nearly de Sitter spacetime assumes a standard form in terms of conformal time $\mathrm{d} \tau=\mathrm{d} t / a(t)$,

$$
\left\langle\mathbf{T} \delta \phi^{\alpha}(\mathbf{k}, \tau) \delta \phi^{\beta}\left(\mathbf{k}^{\prime}, \tau^{\prime}\right)\right\rangle=(2 \pi)^{3} \delta\left(\mathbf{k}+\mathbf{k}^{\prime}\right) \delta^{\alpha \beta} \times \begin{cases}f_{k}(\tau) f_{k^{\prime}}^{*}\left(\tau^{\prime}\right) & \tau<\tau^{\prime} \\ f_{k}^{*}(\tau) f_{k^{\prime}}\left(\tau^{\prime}\right) & \tau>\tau^{\prime}\end{cases}
$$

where $f_{k}(\tau)=H(\tau)(1-\mathrm{i} k \tau) \mathrm{e}^{\mathrm{i} k \tau} / \sqrt{2 k^{3}}$ is the elementary wavefunction for a mode of wavenumber $k$. The time-ordering operator T was defined below Eq. (3.6), and we have evaluated the correlation function in the Bunch-Davies vacuum state.

In addition to the condition $(k / a)^{2} \gg m^{2}$, Eq. (6.1) requires that the expansion rate $H(t)$ is sufficiently slowly varying that $\epsilon \equiv-\dot{H} / H^{2} \ll 1$, where the overdot denotes a derivative with respect to cosmic time $t$. Therefore, although the rest of our numerical scheme makes no use of the slow-roll approximation, our use of (6.1) to compute initial conditions does require that $\epsilon$ be at least modestly small at the initial time - and for at least a few prior e-folds in order that each mode has time to relax safely into its vacuum state. ${ }^{10}$ To successfully estimate initial values for the three-point correlation functions we must impose a

\footnotetext{
${ }^{10}$ In general, this means it is not possible to use the initial conditions obtained in this section to study effects very close to the beginning of inflation. A separate prescription is required to study modes that exit the horizon very early in the inflationary phase.
} 
similar slow-variation condition on other background quantities such as the field derivatives $\dot{\phi}^{\alpha}$. Two comments can be made about this restriction. First, while it necessarily involves some loss of flexibility, the requirement that $\epsilon<1$ and $\dot{\phi}^{\alpha}$ is slowly varying is less restrictive than it may appear because we would normally expect to place the initial time during a quiescent phase. Second, although $\epsilon<1$ should be approximately satisfied, we normally do not require the strong condition $\epsilon \ll 1$ provided the slow-roll approximation is close enough to the true solution that it lies within its basin of attraction, and there is enough time for the numerical solution to relax to this 'true' value.

On subhorizon scales $k / a H$ is exponentially large and therefore the highest power of $k \tau_{\text {init }} \approx k /(a H)_{\text {init }}$ is dominant, where the subscript 'init' denotes evaluation at the initial cosmic time $t_{\text {init }}$. From Eq. (6.1) we can deduce all two-point functions containing any combination of fields and momenta. At highest order in $k \tau_{\text {init }}$ and lowest order in $\epsilon$, and neglecting any mass terms, this gives

$$
\begin{aligned}
& \Sigma_{\mathrm{Re}}^{a b}\left(t_{\text {init }}\right)=\frac{1}{2 a^{3} k}\left(\begin{array}{cc}
a \delta^{\alpha \beta} & -a H \delta^{\alpha \beta} \\
-a H \delta^{\alpha \beta} & \left(k^{2} / a\right) \delta^{\alpha \beta}
\end{array}\right) \\
& \Sigma_{\operatorname{Im}}^{a b}\left(t_{\text {init }}\right)=\frac{1}{2 a^{3} k}\left(\begin{array}{cc}
0 & k \delta^{\alpha \beta} \\
k \delta^{\alpha \beta} & 0
\end{array}\right),
\end{aligned}
$$

where all quantities on the right-hand side are to be evaluated at $t_{\text {init }}$.

\subsection{Tensor modes}

Since tensor modes behave like free scalar fields (aside from normalization) their initial conditions follow directly from Eq. (6.2a). Remembering that we need work only with the real part, the required initial condition is

$$
\Gamma^{\mathfrak{a} \mathfrak{b}}\left(t_{\text {init }}\right)=\frac{1}{a^{3} k M_{\mathrm{P}}^{2}}\left(\begin{array}{cc}
a & -a H \\
-a H & k^{2} / a
\end{array}\right),
$$

where $\Gamma^{\mathfrak{a} \mathfrak{b}}$ was defined in Eq. (5.10). As above, all quantities on the right-hand side are to be evaluated at $t_{\text {init. }}$.

\subsection{Three-point function}

To compute an initial condition for the three-point function we use the in-in formula (3.7) at tree-level in conjunction with the Bunch-Davies unequal-time two-point function (6.1). The interaction Hamiltonian $H_{\text {int }}$ has already been computed in Eqs. (4.8a)-(4.8c). As above, it is sufficient to perform the calculation to highest order in $k \tau_{\text {init }}$. This means that we may neglect mass terms in the propagator. The method is the same used in standard calculations of the three-point function [42-44,61], but here we extract a limit long before horizon exit rather than long after.

Example.-Consider the equal-time three-point function $\left\langle\delta \phi^{\alpha}\left(\mathbf{k}_{1}\right) \delta \phi^{\beta}\left(\mathbf{k}_{2}\right) \delta \phi^{\gamma}\left(\mathbf{k}_{3}\right)\right\rangle_{t_{\text {init }}}$. The tree-level calculation involves the third-order vertex functions $A_{\alpha \beta \gamma}, B_{\alpha \beta \gamma}$ and $C_{\alpha \beta \gamma}$ defined in Eqs. (4.8a)-(4.8c). Of these, $A_{\alpha \beta \gamma}$ contains a 'fast' term of order $(k / a)^{2} \sim(k \tau)^{2}$ that grows exponentially on subhorizon scales, whereas the remaining terms in $A_{\alpha \beta \gamma}$, and all terms in 
$B_{\alpha \beta \gamma}$ and $C_{\alpha \beta \gamma}$, are 'slow' and do not grow exponentially. Meanwhile, Eqs. (6.1) and (6.2a)(6.2b) show that on subhorizon scales the wavefunction for a field perturbation grows like $(1-\mathrm{i} k \tau) \mathrm{e}^{\mathrm{i} k \tau} \sim k \tau$, whereas the wavefunction for a momentum perturbation behaves like its $t$ derivative $\sim H k^{2} \tau^{2} \mathrm{e}^{\mathrm{i} k \tau} \cdot{ }^{11}$

These estimates enable us to decide which terms should be retained when computing correlations at $t_{\text {init }}$. For example, on subhorizon scales, the fast part of the $A_{\alpha \beta \gamma}$ vertex integral will scale like

$$
\text { fast } A_{\alpha \beta \gamma} \sim \int_{-\infty^{+}}^{\tau_{\text {init }}} \mathrm{d} \eta a(\eta)^{2} f_{k_{1}}(\eta) f_{k_{2}}(\eta) f_{k_{3}}(\eta) \sim \int_{-\infty^{+}}^{\tau_{\mathrm{init}}} \frac{\mathrm{d} \eta}{H^{2} \eta^{2}} k_{1} k_{2} k_{3} \eta^{3} \mathrm{e}^{\mathrm{i} k t \eta}
$$

The $\eta$ integral can be estimated by the usual method of asymptotic expansion $[42,53,61,105]$, yielding

$$
\int_{-\infty^{+}}^{\tau_{\text {init }}} \mathrm{d} \eta \eta \mathrm{e}^{\mathrm{i} k_{t} \eta}=-\frac{\mathrm{i}}{k_{t}^{3}}\left(k_{t} \tau_{\text {init }} \mathrm{e}^{\mathrm{i} k_{t} \tau_{\text {init }}}+\mathrm{O}\left(k_{t} \tau_{\text {init }}\right)^{0}\right) .
$$

There is no contribution from the lower limit of integration because it is killed by the contour deformation prescription that implements adiabatic switch-on of the interaction. For sufficiently early $t_{\text {init }}$ we need retain only the term proportional to $\tau_{\text {init }}$ in Eq. (6.5). By comparison any lower-order terms are exponentially suppressed.

The vertex integral for the slow part of $A_{\alpha \beta \gamma}$ yields terms of order $\mathrm{O}\left(k_{t} \tau_{\text {init }}\right)^{-1}$, making it negligible in many models. However, these slow terms must be included in QSFI-like scenarios where $V_{\alpha \beta \gamma} / H \sim 1$ and therefore $A_{\alpha \beta \gamma} \sim H$. This generates a large contribution that can compensate for suppression by $\left(k_{t} \tau_{\text {init }}\right)^{2}$, at least if $\tau_{\text {init }}$ is selected to lie a modest number of e-folds before horizon exit. We will see in $\S 10.1$ that this is usually the case for practical numerical work. The conclusion is that, in QSFI-like scenarios, both fast and slow contributions to the initial condition from $A_{\alpha \beta \gamma}$ are comparable.

To accommodate this, it is safest to retain the highest power of $k_{t} \tau_{\text {init }}$ from each of the four distinct contributions (fast- $A$, slow- $A, B, C$ ). In simple models the highest dimension operators are typically most relevant, and therefore the fast- $A$ and $C$-type contributions dominate the initial condition. These are dimension-5 operators. In QSFI-type models the slow- $A$ (dimension-3) contributions are also relevant due to their large coupling, as explained above. In most models the $B$-type (dimension-4) contributions are irrelevant, but they are easily kept and therefore we retain them as a precaution. This allows us to successfully handle models where a tuned combination of couplings makes them competitive with the dimension-5 contributions.

To illustrate the method we continue with dimension-5 operators only, although our final results (6.7a)-(6.7d) contain contributions from operators of all types. When $\left|k_{i} \tau\right| \gg 1$ for all relevant scales $k_{i}$, the dimension-5 contribution to the initial condition is

$$
\begin{aligned}
B_{\mathrm{Re}, \mathrm{dim}-5}^{\alpha \beta \gamma} \approx & \frac{\mathrm{i} H_{\text {init }}^{3}}{8\left(k_{1} k_{2} k_{3}\right)} \tau_{\text {init }}^{3} \mathrm{e}^{-\mathrm{i} k_{t} \tau_{\text {init }}} \int_{-\infty^{+}}^{\tau_{\text {init }}} \mathrm{d} \eta H \eta \mathrm{e}^{\mathrm{i} k_{t} \eta}\left(\frac{\pi^{\alpha}}{4 H M_{\mathrm{P}}^{2}} \delta^{\beta \gamma}\left(\mathbf{k}_{2} \cdot \mathbf{k}_{3}\right)-\frac{C^{\alpha \beta \gamma}}{2} k_{1} k_{2}\right) \\
& +5 \text { perms }+ \text { c.c. },
\end{aligned}
$$

\footnotetext{
${ }^{11}$ Recall that in the interaction picture we should use the relation $\delta \pi^{\alpha}=\delta \dot{\phi}^{\alpha}$ to express the momentum perturbation in terms of the creation and annihilation operators for $\delta \phi^{\alpha}$, and not Eq. (4.13).
} 
where the permutations are produced by simultaneous cyclic exchange of the indices $\alpha, \beta, \gamma$ and their corresponding wavevectors $\mathbf{k}_{1}, \mathbf{k}_{2}, \mathbf{k}_{3}$, and 'c.c.' denotes the complex conjugate of the entire expression. ${ }^{12}$ Note that this makes the tree-level three-point function automatically real.

If the time-dependent background quantities $H, \dot{\phi}^{\alpha}$ (and so on) are slowly varying near

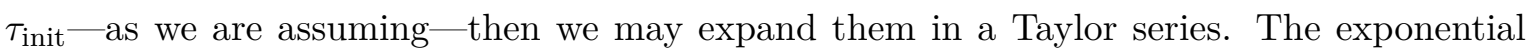
decay of the integrand produced by the contour deformation at large $|\eta|$ implies that highorder terms in the Taylor expansion make small contributions to the integral $[53,105]$, and to leading order we need only the first term. In practice this means we can evaluate all background terms at $\tau_{\text {init }}$ and take them outside the $\eta$ integral.

Results.- Repeating this exercise for each correlation function we find the following results. In each case we quote the correlation function as a initial term plus a number of permutations over simultaneous cyclic exchange of $\alpha, \beta, \gamma$ and $\mathbf{k}_{1}, \mathbf{k}_{2}, \mathbf{k}_{3}$, as above. Notice that the permutations should only be made within the bracket in which the sum appears; not all terms should be symmetrized, because momentum factors from the external wavefunctions may be asymmetric in mixed field-momentum correlation functions. The species labels $\alpha, \beta$, $\gamma$ correspond to the phase-space labels $a, b, c$, and $A_{\text {slow }}^{\alpha \beta \gamma}$ is defined to contain only the slow terms in $A^{\alpha \beta \gamma}$. We set $k_{t} \equiv k_{1}+k_{2}+k_{3}$ equal to the perimeter of the momentum triangle and use the abbreviation $K^{2} \equiv k_{1} k_{2}+k_{1} k_{3}+k_{2} k_{3}$.

- $a, b, c$ all fields

$$
\begin{aligned}
B_{\mathrm{Re}}^{a b c}\left(t_{\text {init }}\right)=\frac{1}{4 a\left(t_{\text {init }}\right)^{4}} \frac{1}{k_{1} k_{2} k_{3} k_{t}}( & \frac{\pi^{\alpha}}{4 H M_{\mathrm{P}}^{2}} \delta^{\beta \gamma} \mathbf{k}_{2} \cdot \mathbf{k}_{3}-\frac{1}{2} C^{\alpha \beta \gamma} k_{1} k_{2}+\frac{a^{2}}{2} A_{\text {slow }}^{\alpha \beta \gamma} \\
& \left.+\frac{a^{2} H}{2} B^{\alpha \beta \gamma}\left[\frac{\left(k_{1}+k_{2}\right) k_{3}}{k_{1} k_{2}}-\frac{K^{2}}{k_{1} k_{2}}\right]+5 \text { perms }\right)_{t=t_{\text {init }}}
\end{aligned}
$$

We can estimate the size of the dimension-5 contributions from $C^{\alpha \beta \gamma} k_{1} k_{2}$, which is roughly $k^{2} / M_{\mathrm{P}}$ assuming all $k_{i}$ are close to a common scale $k$. Meanwhile, the slow- $A$ contribution is $a^{2} A^{\alpha \beta \gamma}$. In a QSFI-like model this is $\sim a^{2} H$. The relative importance of the slow- $A$ terms is then roughly $\left(a^{2} H^{2} / k^{2}\right)\left(M_{\mathrm{P}} / H\right) \sim\left(k \tau_{\text {init }}\right)^{-2}\left(M_{\mathrm{P}} / H\right)$, confirming the conclusion above that these contributions can be comparable if $t_{\text {init }}$ is not too early.

\section{- $a$ momentum, $b, c$ fields}

\footnotetext{
${ }^{12}$ In this section we are not making use of the extended summation convention described on p.13, and therefore we revert to labelling the momenta as $\mathbf{k}_{1}, \mathbf{k}_{2}, \mathbf{k}_{3}$ to avoid ambiguity in combinations such as $C^{\alpha \beta \gamma} k_{1} k_{2}$.
} 


$$
\begin{aligned}
& B_{\mathrm{Re}}^{a b c}\left(t_{\text {init }}\right)=\frac{1}{4 a\left(t_{\text {init }}\right)^{4}} \frac{1}{\left(k_{1} k_{2} k_{3}\right)^{2} k_{t}} \\
& \times\left(k_{1}^{2}\left(k_{2}+k_{3}\right)\left[\frac{\pi^{\alpha}}{4 H M_{\mathrm{P}}^{2}} \delta^{\beta \gamma} \mathbf{k}_{2} \cdot \mathbf{k}_{3}+\frac{a^{2}}{2} A_{\text {slow }}^{\alpha \beta \gamma}-\frac{1}{2} C_{\alpha \beta \gamma} k_{1} k_{2}+5 \text { perms }\right]\right. \\
& +k_{1}\left[\frac{k_{1}^{2} k_{2}^{2}}{2}\left(1+\frac{k_{3}}{k_{t}}\right) C^{\alpha \beta \gamma}+\frac{1}{2 H} B^{\alpha \beta \gamma} k_{1} k_{2} k_{3}^{2}-\frac{\pi^{\alpha}}{4 H M_{\mathrm{P}}^{2}} \delta^{\beta \gamma}\left(K^{2}+\frac{k_{1} k_{2} k_{3}}{k_{t}}\right) \mathbf{k}_{2} \cdot \mathbf{k}_{3}\right. \\
& \left.\left.\quad-\frac{a^{2}}{2} A_{\text {slow }}^{\alpha \beta \gamma}\left(K^{2}-\frac{k_{1} k_{2} k_{3}}{k_{t}}\right)+5 \text { perms }\right]\right)_{t=t_{\text {init }}}
\end{aligned}
$$

- $a, b$ momenta, $c$ field

$$
\begin{aligned}
& B_{\operatorname{Re}}^{a b c}\left(t_{\text {init }}\right)=\frac{1}{4 a\left(t_{\text {init }}\right)^{6}} \frac{1}{\left(k_{1} k_{2} k_{3}\right)^{2} k_{t} H\left(t_{\text {init }}\right)^{2}} \\
& \times\left(k _ { 1 } ^ { 2 } k _ { 2 } ^ { 2 } k _ { 3 } \left[\frac{1}{2} C^{\alpha \beta \gamma} k_{1} k_{2}-\frac{\pi^{\alpha}}{4 H M_{\mathrm{P}}^{2}} \delta^{\beta \gamma} \mathbf{k}_{2} \cdot \mathbf{k}_{3}-\frac{a^{2}}{2} A_{\text {slow }}^{\alpha \beta \gamma}-\frac{a^{2} H}{2} B^{\alpha \beta \gamma} \frac{\left(k_{1}+k_{2}\right) k_{3}}{k_{1} k_{2}}\right.\right. \\
& \left.\quad+5 \text { perms }]+k_{1}^{2} k_{3}^{2}\left[\frac{a^{2} H}{2} B^{\alpha \beta \gamma} k_{3}+5 \text { perms }\right]\right)_{t=t_{\text {init }}}
\end{aligned}
$$

- $a, b, c$ all momenta

$$
\begin{aligned}
B_{\mathrm{Re}}^{a b c}\left(t_{\text {init }}\right)=\frac{1}{4 a\left(t_{\text {init }}\right)^{6}} \frac{1}{k_{1} k_{2} k_{3} k_{t} H\left(t_{\text {init }}\right)^{2}}( & \frac{\pi^{\alpha}}{4 H M_{\mathrm{P}}^{2}} \delta^{\beta \gamma}\left[K^{2}+\frac{k_{1} k_{2} k_{3}}{k_{t}}\right] \mathbf{k}_{2} \cdot \mathbf{k}_{3}-\frac{1}{2 H} B_{\beta \gamma \alpha} k_{1}^{2} k_{2} k_{3} \\
& -\frac{k_{1}^{2} k_{2}^{2}}{2}\left[1+\frac{k_{3}}{k_{t}}\right] C^{\alpha \beta \gamma}+\frac{a^{2}}{2} A_{\text {slow }}^{\alpha \beta \gamma}\left[K^{2}-\frac{k_{1} k_{2} k_{3}}{k_{t}}\right] \\
& +5 \text { perms })_{t=t_{\text {init }}}
\end{aligned}
$$

Once these initial conditions have been obtained, their subsequent evolution takes place on the real time axis. As explained above, the evolution can therefore be implemented by ordinary differential equations without any requirement to account for contour rotation.

\section{Gauge transformation to the curvature perturbation}

The formalism developed in $\S \S 3-6$ yields a numerical scheme suitable to compute the phasespace correlation functions $\left\langle\delta X^{\mathrm{a}} \delta X^{\mathrm{b}}\right\rangle$ and $\left\langle\delta X^{\mathrm{a}} \delta X^{\mathrm{b}} \delta X^{\mathrm{c}}\right\rangle$. However, to construct inflationary observables we are normally interested in the correlation functions of the curvature perturbation $\langle\zeta \zeta\rangle$ and $\langle\zeta \zeta \zeta\rangle .{ }^{13}$ Since $\zeta$ and the $\delta X^{a}$ are related by a nonlinear gauge transformation

\footnotetext{
${ }^{13}$ There are other possible observables of interest, such as those related to isocurvature perturbations. In this paper we focus on $\zeta$, but it is straightforward to compute correlation functions for isocurvature modes using the transport framework. In the same way that $\zeta$ can be regarded as a scaled projection along the instantaneous background trajectory, isocurvature perturbations correspond to projections onto directions orthogonal to it. We do not pursue the details here; see the discussion in Ref. [35].
} 
the same is true for their correlation functions. To compute the two- and three-point functions of $\zeta$ we need the details of this gauge transformation up to second order in $\delta X^{\mathrm{a}}$. Suitable expressions were obtained in Ref. [106].

It is possible to present the result in a number of different ways, each of which are related by constraint equations. For our purposes, a convenient real-space form is

$$
\begin{aligned}
\zeta=-\frac{\dot{\phi}^{\alpha} \delta \phi_{\alpha}}{2 H M_{\mathrm{P}}^{2} \epsilon}+\frac{1}{6 H^{2} M_{\mathrm{P}}^{2} \epsilon} & \left(\frac{\dot{\phi}_{\alpha} \dot{\phi}_{\beta}}{M_{\mathrm{P}}^{2}}\left[-\frac{3}{2}+\frac{9}{2 \epsilon}+\frac{3}{4 \epsilon^{2}} \frac{V_{\gamma} \dot{\phi}^{\gamma}}{M_{\mathrm{P}}^{2} H^{3}}\right] \delta \phi^{\alpha} \delta \phi^{\beta}\right. \\
& \left.+\frac{3}{H \epsilon} \frac{\dot{\phi}_{\alpha} \dot{\phi}_{\beta}}{M_{\mathrm{P}}^{2}} \delta \phi^{\alpha} \delta \dot{\phi}^{\beta}-3 H \partial^{-2}\left[\partial_{j} \delta \dot{\phi}^{\alpha} \partial_{j} \delta \phi_{\alpha}+\delta \dot{\phi}^{\alpha} \partial^{2} \delta \phi_{\alpha}\right]\right) .
\end{aligned}
$$

All quantities in this relation should be evaluated at the same time, corresponding to the time of evaluation for $\zeta$.

The transformation (7.1) is expressed in terms of time derivatives $\delta \dot{\phi}^{\alpha}$ rather than momenta $\delta \pi^{\alpha}$. To account for this we must either exchange the phase-space correlation functions $\left\langle\delta X^{\mathrm{a}} \delta X^{\mathrm{b}}\right\rangle,\left\langle\delta X^{\mathrm{a}} \delta X^{\mathrm{b}} \delta X^{\mathrm{c}}\right\rangle$ for correlation functions of $\delta \phi^{\alpha}, \delta \dot{\phi}^{\alpha}$, or use Eq. (4.13) to rewrite (7.1) in terms of the $\delta \pi^{\alpha}$. If we adopt this second strategy then Eq. (7.1) is an especially simple representation because the absence of a linear term in $\delta \dot{\phi}^{\alpha}$ means that the second-order parts of (4.13) are not needed.

Following this procedure and reverting to our extended summation convention, the gauge transformation can be written

$$
\zeta(\mathbf{k})=N_{\mathrm{a}}(\mathbf{k}) \delta X^{\mathrm{a}}+\frac{1}{2} N_{\mathrm{ab}}(\mathbf{k}) \delta X^{\mathrm{a}} \delta X^{\mathrm{b}} .
$$

In order to give a complete set of equations we quote results for $N_{\mathrm{a}}$ and $N_{\mathrm{ab}}$ explicitly. We write

$$
\begin{aligned}
N_{\mathrm{a}}(\mathbf{k}) & =(2 \pi)^{3} \delta\left(\mathbf{k}-\mathbf{k}_{a}\right) N_{a} \\
N_{\mathrm{ab}}(\mathbf{k}) & =(2 \pi)^{3} \delta\left(\mathbf{k}-\mathbf{k}_{a}-\mathbf{k}_{b}\right) N_{a b}\left(\mathbf{k}, \mathbf{k}_{a}, \mathbf{k}_{b}\right) .
\end{aligned}
$$

The coefficient matrices $N_{a}$ and $N_{a b}$ satisfy

$$
\begin{aligned}
N_{a} & =-\frac{\pi_{\alpha}}{2 H M_{\mathrm{P}}^{2} \epsilon}\left(\begin{array}{c}
1 \\
0
\end{array}\right) \\
N_{a b} & =\frac{1}{3 H^{2} M_{\mathrm{P}}^{2} \epsilon}\left(\begin{array}{cc}
\frac{\pi_{\alpha} \pi_{\beta}}{M_{\mathrm{P}}^{2}}\left[-\frac{3}{2}+\frac{9}{2 \epsilon}+\frac{3}{4 \epsilon^{2}} \frac{V_{\gamma} \pi^{\gamma}}{M_{\mathrm{P}}^{2} H^{3}}\right] & \frac{3}{H \epsilon} \frac{\pi_{\alpha} \pi_{\beta}}{M_{\mathrm{P}}^{2}}-\frac{3 H}{k^{2}}\left[\mathbf{k}_{a} \cdot \mathbf{k}_{b}+k_{a}^{2}\right] \delta_{\alpha \beta} \\
\frac{3}{H \epsilon} \frac{\pi_{\alpha} \pi_{\beta}}{M_{\mathrm{P}}^{2}}-\frac{3 H}{k^{2}}\left[\mathbf{k}_{a} \cdot \mathbf{k}_{b}+k_{b}^{2}\right] \delta_{\alpha \beta} & 0
\end{array}\right)
\end{aligned}
$$

Notice that, in this representation, $N_{a}$ is independent of wavenumber. We define the spectrum and bispectrum of $\zeta$ by the rules

$$
\begin{aligned}
\left\langle\zeta\left(\mathbf{k}_{1}\right) \zeta\left(\mathbf{k}_{2}\right)\right\rangle & =(2 \pi)^{3} \delta\left(\mathbf{k}_{1}+\mathbf{k}_{2}\right) P(k) \\
\left\langle\zeta\left(\mathbf{k}_{1}\right) \zeta\left(\mathbf{k}_{2}\right) \zeta\left(\mathbf{k}_{3}\right)\right\rangle & =(2 \pi)^{3} \delta\left(\mathbf{k}_{1}+\mathbf{k}_{2}+\mathbf{k}_{3}\right) B\left(k_{1}, k_{2}, k_{3}\right) .
\end{aligned}
$$


To compute these from the phase-space correlation functions we should use (assuming the time of evaluation to be sufficiently late that any imaginary components of the two-point function have decayed)

$$
\begin{aligned}
P(k) & =N_{a} N_{b} \Sigma_{\operatorname{Re}}^{a b}(k) \\
B\left(k_{1}, k_{2}, k_{3}\right) & =N_{a} N_{b} N_{c} B_{\operatorname{Re}}^{a b c}\left(k_{1}, k_{2}, k_{3}\right)+\left(N_{a} N_{b} N_{c d}\left(\mathbf{k}_{3}, \mathbf{k}_{1}, \mathbf{k}_{2}\right) \Sigma_{\operatorname{Re}}^{a c}\left(k_{1}\right) \Sigma_{\operatorname{Re}}^{b d}\left(k_{2}\right)+2 \text { cyclic }\right) .
\end{aligned}
$$

Tensor fraction.-The tensor power spectrum is defined by analogy with the scalar spectrum,

$$
\left\langle\gamma_{i j}\left(\mathbf{k}_{1}\right) \gamma^{i j}\left(\mathbf{k}_{2}\right)\right\rangle \equiv(2 \pi)^{3} \delta\left(\mathbf{k}_{1}+\mathbf{k}_{2}\right) P_{\gamma} .
$$

Using the normalization condition $\operatorname{tr}\left(\boldsymbol{e}^{s} \cdot \boldsymbol{e}^{s^{\prime}}\right)=2 \delta^{s s^{\prime}}$ it follows that

$$
\left\langle\gamma_{i j}\left(\mathbf{k}_{1}\right) \gamma^{i j}\left(\mathbf{k}_{2}\right)\right\rangle=\sum_{s} \sum_{s^{\prime}}\left\langle\gamma_{s}\left(\mathbf{k}_{1}\right) \gamma_{s}\left(\mathbf{k}_{2}\right)\right\rangle \operatorname{tr}\left(\boldsymbol{e}^{s} \cdot \boldsymbol{e}^{s^{\prime}}\right)=2 \sum_{s}\left\langle\gamma_{s}\left(\mathbf{k}_{1}\right) \gamma_{s}\left(\mathbf{k}_{2}\right)\right\rangle
$$

Each polarization has the same amplitude and therefore the final result is $P_{\gamma}=4\left\langle\gamma_{+} \gamma_{+}\right\rangle=$ $4\left\langle\gamma_{\times} \gamma_{\times}\right\rangle$. The tensor-to-scalar ratio $r$ is defined by

$$
r \equiv \frac{P_{\gamma}}{P_{\zeta}}=\frac{4 \Gamma^{\gamma \gamma}}{P_{\zeta}}
$$

where $\Gamma^{\mathfrak{a} \mathfrak{b}}$ is the tensor two-point function defined in Eq. (5.10).

\section{The PyTransport and CppTransport codes}

We have now assembled the formalism necessary to build automated tools that compute the two- and three-point functions of phase-space perturbations $\delta X^{a}$ or the curvature perturbation $\zeta$. In summary, the steps involved are:

1. Information about the model must be provided - specifically, the number of fields, the form of the potential, the background initial conditions and the total integration time. In PyTransport this is done by writing a Python script that builds the potential as a SymPy expression. In CppTransport it is achieved by writing a separate 'model description file' and passing it to a specialized translation tool.

2. In the case of CppTransport, the automated tool uses this information to symbolically compute the tensors $A_{\alpha \beta \gamma}, B_{\alpha \beta \gamma}$ and $C_{\alpha \beta \gamma}$. These are used to build expressions for $u^{\mathrm{a}}{ }_{\mathrm{b}}$ and $u^{\mathrm{a}} \mathrm{bc}$. In the case of PyTransport the automated tool symbolically computes only the derivatives of the potential.

3. In CppTransport these symbolic expressions are used to construct specialized $\mathrm{C}++$ code that solves the ordinary differential equations (5.5) and (5.16). Further specialized code is generated to compute the initial conditions (6.2a)-(6.2b) and (6.7a)-(6.7d), and the gauge transformation matrices $(7.4 \mathrm{a})-(7.4 \mathrm{~b})$. 
This specialized code is generated by the translation tool. The platform provides an object-based interface that is used to define specific tasks, such as the computation of an $n$-point function over a fixed range of wavenumber configurations. These tasks are written into a database, after which they can be executed. No specific logic is needed to control how the calculation is performed.

In PyTransport specialized $\mathrm{C}++$ code to compute the derivatives of the potential is generated by a Python script. The tensors $A_{\alpha \beta \gamma}, B_{\alpha \beta \gamma}$ and $C_{\alpha \beta \gamma}$ and all other quantities are hard-coded in terms of the potential and its derivatives. Finally, the $\mathrm{C}++$ code is compiled into a Python module and thereafter can be included in further Python scripts that use its functionality to compute $n$-point functions.

4. To solve for a specific $n$-point function we should determine how early it is necessary to set the initial conditions in order that the massless approximation is sufficiently good. In CppTransport this calculation is automated, whereas in PyTransport Python scripts are provided to perform this task. The compiled $\mathrm{C}++$ code is used to produce suitable initial conditions and then to evolve the correlation functions until some specified final time.

Computation of the three-point function requires knowledge of the background inflationary trajectory and the two-point functions that act as source terms in Eq. (5.16). In principle both of these could be pre-computed over the required range of scales, but in practice the integration time is normally dominated by evolution of the three-point function. Therefore we simultaneously evolve the background together with the real and imaginary parts of the two-point function for each of the scales $k_{1}, k_{2}, k_{3}$.

5. Eqs. (7.4a)-(7.4b) are used to convert the phase-space correlation functions into $\zeta$ correlators. At this stage it is possible to extract derived quantities such as the spectral index of the power spectrum $P(k)$ or the reduced bispectrum $(6 / 5) f_{\mathrm{NL}}\left(k_{1}, k_{2}, k_{3}\right)=$ $B\left(k_{1}, k_{2}, k_{3}\right) /\left[P\left(k_{1}\right) P\left(k_{2}\right)+P\left(k_{1}\right) P\left(k_{3}\right)+P\left(k_{2}\right) P\left(k_{3}\right)\right]$. Alternatively the $\zeta$ bispectrum can be used to compute an amplitude associated with one of the standard bispectrum shapes such as the 'local' or 'equilateral' templates.

Obtaining the codes.-PyTransport and CppTransport can be downloaded from the website transportmethod.com. Additionally, releases of both platforms are archived at the zenodo.org repository. At the time of writing the current version of PyTransport is v1.0 and the current version of CppTransport is 2016.3.

CppTransport can alternatively be downloaded from the development repository hosted at GitHub. This makes it possible to obtain pre-release versions of the software.

Plots included in this paper.- - In $\S 9$ we give a number of examples that illustrate the usefulness of our framework, and the capabilities of the PyTransport and CppTransport platforms specifically. In each case we have verified that both platforms give equivalent results, but the plots we include were generated by CppTransport. 
- For CppTransport the plots were generated using the 2016.3 release, or platform revision 36ac30da. Source code for the plots can be obtained from a separate GitHub repository using revision 7ef3852f.

- For PyTransport the plots were generated using platform revision release version 1.0, and are stored in a folder that accompanies the release. The code used to generate the plots is contained in subfolders of the Examples folder that accompanies the release.

\section{$9 \quad$ Numerical Examples}

In this section we illustrate the utility of our method by computing the two- and three-point functions for a collection of example theories.

There are several ways to present numerical solutions for the spectrum and bispectrum. For the two-point function we normally plot the 'dimensionless' spectrum $\mathcal{P}$, defined by

$$
\mathcal{P}(k)=\frac{k^{3}}{2 \pi^{2}} P(k),
$$

where $P(k)$ is the $\zeta$ power spectrum defined in Eq. (7.6a). For the three-point function we plot either the dimensionless bispectrum

$$
\mathcal{B}\left(k_{1}, k_{2}, k_{3}\right)=\left(k_{1} k_{2} k_{3}\right)^{2} B\left(k_{1}, k_{2}, k_{3}\right),
$$

or the 'reduced bispectrum',

$$
\frac{6}{5} f_{\mathrm{NL}}\left(k_{1}, k_{2}, k_{3}\right)=\frac{B\left(k_{1}, k_{2}, k_{3}\right)}{P\left(k_{1}\right) P\left(k_{2}\right)+P\left(k_{1}\right) P\left(k_{3}\right)+P\left(k_{2}\right) P\left(k_{3}\right)} .
$$

The dimensionless bispectrum is sometimes called the 'shape function' (up to an irrelevant normalization) and denoted $S$ [107]. In both (9.2) and (9.3) $B\left(k_{1}, k_{2}, k_{3}\right)$ is the $\zeta$ bispectrum of Eq. (7.6b). The dimensionless bispectrum is a measure of the three-point function alone, whereas the reduced bispectrum measures the relative nonlinearity between the two- and three-point functions. If the bispectrum is generated by a quadratic contribution $\zeta=\zeta_{g}+$ $(3 / 5) f_{\mathrm{NL}}^{\text {local }} \zeta_{g}^{2}$ (where $\zeta_{g}$ is a Gaussian random field) then $f_{\mathrm{NL}}\left(k_{1}, k_{2}, k_{3}\right)$ is equal to $f_{\mathrm{NL}}^{\text {local }}$, but more generally it will depend on the wavenumber configuration.

To express this configuration dependence it is convenient to distinguish between the scale and shape of the triangle $\mathbf{k}_{1}+\mathbf{k}_{2}+\mathbf{k}_{3}$ formed from the individual wavenumbers. The scale can be measured by the perimeter $k_{t}=k_{1}+k_{2}+k_{3}$ of the triangle. To measure the shape we use the parameters $\alpha$ and $\beta$ introduced by Fergusson \& Shellard [108],

$$
\begin{aligned}
& k_{1}=\frac{k_{t}}{4}(1+\alpha+\beta) \\
& k_{2}=\frac{k_{t}}{4}(1-\alpha+\beta) \\
& k_{3}=\frac{k_{t}}{2}(1-\beta)
\end{aligned}
$$

The allowed values of $(\alpha, \beta)$ fall inside the triangle with vertices $(-1,0),(1,0)$ and $(0,1)$. At fixed $k_{t}$ isosceles configurations have $\alpha=0$ and the equilateral configuration is $\alpha=0$, $\beta=1 / 3$. Finally, the vertices correspond to 'squeezed limits' where one $k_{i}$ becomes much smaller than the other two. 


\subsection{Axion-quartic model: local-mode bispectrum}

The axion-quartic model was introduced by Elliston et al. [109] as an analytically tractable proxy for a large- $N$ N-flation model where some fields have initial conditions close to the hilltop of an axionic potential $[110,111]$. In this region the potential has a large negative $\eta$ parameter that is communicated to the final bispectrum, even though the slow-roll parameter $\epsilon=-\dot{H} / H^{2}$ and its derivatives are small. The model produces a mostly local-mode bispectrum with $f_{\mathrm{NL}}^{\text {local }} \sim \mathrm{O}(10)$.

This is a two-field model with potential

$$
V=\frac{1}{4} g \phi^{4}+\Lambda^{2}\left(1-\cos \frac{2 \pi \chi}{f}\right) .
$$

The field $\chi$ represents the degree of freedom whose initial position lies closest to the cosine hilltop, whereas $\phi$ represents the aggregate effect of the other fields [109]. A similar potential had been studied earlier in Ref. [52], but Eq. (9.5) has the advantage of producing a spectrum with acceptable tilt. In our numerical results we choose $g=10^{-10}, \Lambda^{4}=(25 / 2 \pi)^{2} g M_{\mathrm{P}}^{4}$ and $f=M_{\mathrm{P}}$. The initial conditions are $\phi=23.5 M_{\mathrm{P}}$ and $\chi=f / 2-10^{-3} M_{\mathrm{P}}$, and the initial field derivatives are determined by the slow-roll approximation.

Bispectrum shape.- In Fig. 5 we plot the evolution of the bispectrum shape as the wavenumbers move from subhorizon to superhorizon scales. The panels show the dimensionless bispectrum $\mathcal{B}\left(k_{1}, k_{2}, k_{3}\right)$ as a function of the shape parameters $\alpha$ and $\beta$ at fixed $k_{t}$, scaled to have unit amplitude at the equilateral point [107]. The value of $k_{t}$ is chosen so that the time of horizon exit for $k_{t} / 3$ is 14.0 e-folds later than the initial conditions. Notice that these plots have a six-fold redundancy corresponding to the 3 ! permutations of the momenta $\mathbf{k}_{1}, \mathbf{k}_{2}, \mathbf{k}_{3}$ that leave $\left\langle\zeta\left(\mathbf{k}_{1}\right) \zeta\left(\mathbf{k}_{2}\right) \zeta\left(\mathbf{k}_{3}\right)\right\rangle$ invariant. Therefore only one-sixth region of each triangle contains independent information, although we follow Ref. [107] in plotting the whole triangle to aid visualization.

The top panel shows the shape at early times, where the short wavenumbers represented by points near the centre of the triangle are on subhorizon scales and the correlations should effectively be those of quantum fluctuations in Minkowski space. The squeezed limits near $(\alpha, \beta)=(1,0),(-1,0)$ and $(0.1)$ are nearly empty because long-short correlations are absent. Only short-short correlations are significant, giving the bispectrum an 'equilateral-like' appearance peaking around the equilateral configuration $(\alpha, \beta)=(0,1 / 3)$.

Eventually, the central short modes approach their horizon exit point and become aware of the cosmological background. At this time long-short correlations begin to develop, pushing up the amplitude near the squeezed limits; see middle panel. (The earliest stages of this process are already visible in the top panel, where there is a tiny upturn near the vertices of the triangle.) Finally, when the short modes are well outside the horizon, the bispectrum approaches the 'local-like' shape shown in the bottom panel.

Our numerical method is capable of capturing very subtle effects in the bispectrum shape. In Fig. 6 we highlight these effects by masking away regions near the vertices, leaving only the central region of the $(\alpha, \beta)$ triangle. As in Fig. 5, the top panel shows the shape at very early times. Notice that contours of equal amplitude are roughly triangular, with 
rounded corners. The overall appearance is smooth but there is a delicate 'wrinkled' structure inherited from the momentum dependence of the vacuum fluctuations.

The central panel captures a transition occurring near $N=57.1$ e-folds from the initial conditions. (The time of evaluation for this panel is not the same as in Fig. 5.) At this time the central short modes are far outside the horizon and the bispectrum amplitude is preparing to undergo rapid changes as the $\chi$ field, previously frozen by Hubble friction, rolls towards its minimum. Although the bispectrum shape is still dominated by the local-like spikes at each vertex, subtle bumps appear near the folded configurations along each edge. Meanwhile, in the central region, a pattern of oscillations is clearly visible, inherited from interference effects around horizon exit.

Finally, the bottom panel shows the configuration at late times when the system has stabilized near an adiabatic limit. The equilateral configuration $(\alpha, \beta)=(0,1 / 3)$ is now a minimum of the amplitude rather than a maximum, and the surrounding contours are much more circular than in the top panel. The central region is very smooth, with the oscillations visible in the middle panel having been damped away.

Time evolution.-Alternatively, we can study the time history of the correlation functions for a single configuration. As we now explain, this is often a useful tool with which to detect contamination of the numerical solution.

In Fig. 7 we plot the evolution of the field-space and $\zeta$ three-point functions for an equilateral configuration with $k_{t}$ adjusted so that $k_{t} / 3$ exits the horizon 14.0 e-folds after the initial time; this configuration corresponds to the equilateral point in Figs. 5 and 6 . We also plot the two-point functions for the scale $k_{t} / 3$ that appears on each side of the momentum triangle.

The critical property appearing in these plots is the smooth, exponential decay of the correlation functions on subhorizon scales. The presence of this feature is easy to understand by comparison with the classical result (6.1) for the two-point function of a massless scalar field in de Sitter space. Specializing to the equal-time limit, the corresponding dimensionless power spectrum is

$$
\mathcal{P}=\frac{H^{2}}{4 \pi^{2}}\left(1+k^{2} \tau^{2}\right)=\frac{H^{2}}{4 \pi^{2}}\left(1+\frac{k^{2}}{a^{2} H^{2}}\right)
$$

where we have used the approximate relation $a=-(H \tau)^{-1}$. If $H$ is almost constant then $(k / a H)^{2} \propto \mathrm{e}^{-2 N}$, reproducing the exponential decay visible in Fig. 7. For the three-point function the decay can be faster if a higher power of $k / a H$ is dominant; cf. the bottom left panel of Fig. 7, where the blue line drops more steeply. Our procedure for computing initial conditions in $\S 6$ amounted to keeping only the exponentially largest terms at early times.

In $\S 10$ we will see that it is very expensive to integrate during this exponentiallydecaying subhorizon phase. This is a problem that afflicts all attempts to numerically solve the inflationary perturbations. In a traditional 'Feynman calculus' code such as FieldInf or ModeCode/MultiModeCode it appears through the obligation to track exponentially rapid phase oscillations $\sim \mathrm{e}^{\mathrm{i} k / a H}$ of the constituent wavefunctions in (6.1). Because our approach is based on evolution of correlation functions rather than their constituent wavefunctions these explicit phase oscillations are absent, but their influence remains through the nonzero imag- 

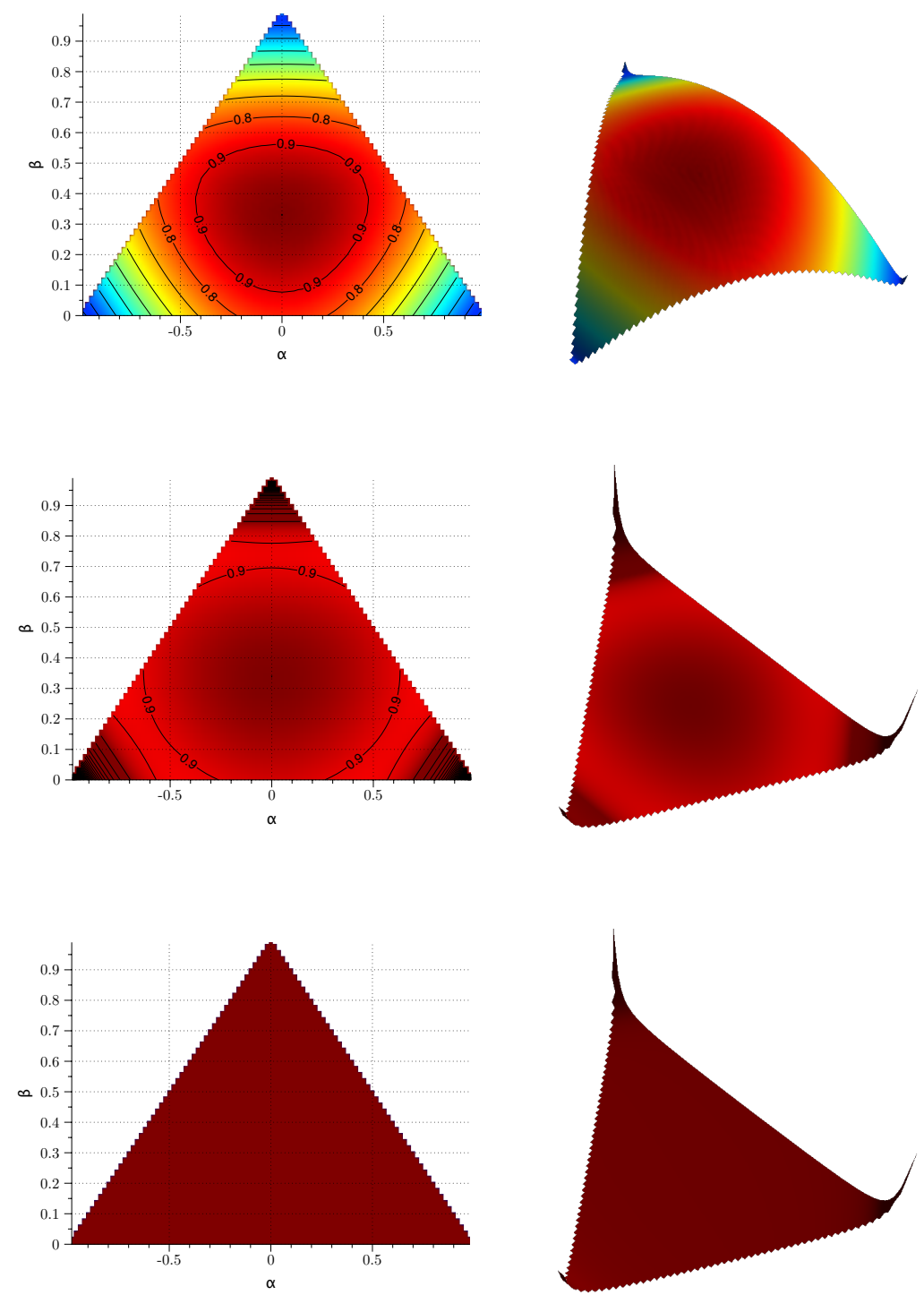

Figure 5. Evolution of the dimensionless bispectrum $\mathcal{B}(\alpha, \beta)$ at fixed $k_{t}$ in the axion-quartic model (9.5).

inary parts of the two-point function that couple off-diagonal terms in our matrix equations. In either case, the net result is that the integrator must dramatically reduce its step size in order to accurately reproduce this smooth exponential profile.

This property is unfortunate from the perspective of pure numerical performance, but it can be exploited to provide a sanity check on the accuracy of the numerical solution. Even small discrepancies can disrupt the cancellations that conspire to produce smooth decay. An example can be seen in the top-left panel of Fig. 7 where the blue line representing the $\phi \chi$ cross-correlation function shows small oscillations at early times. We believe these oscillations stem from a small inaccuracy in the massless approximation, which remains relevant in these correlation functions because of their small amplitude. The initial condition could perhaps 

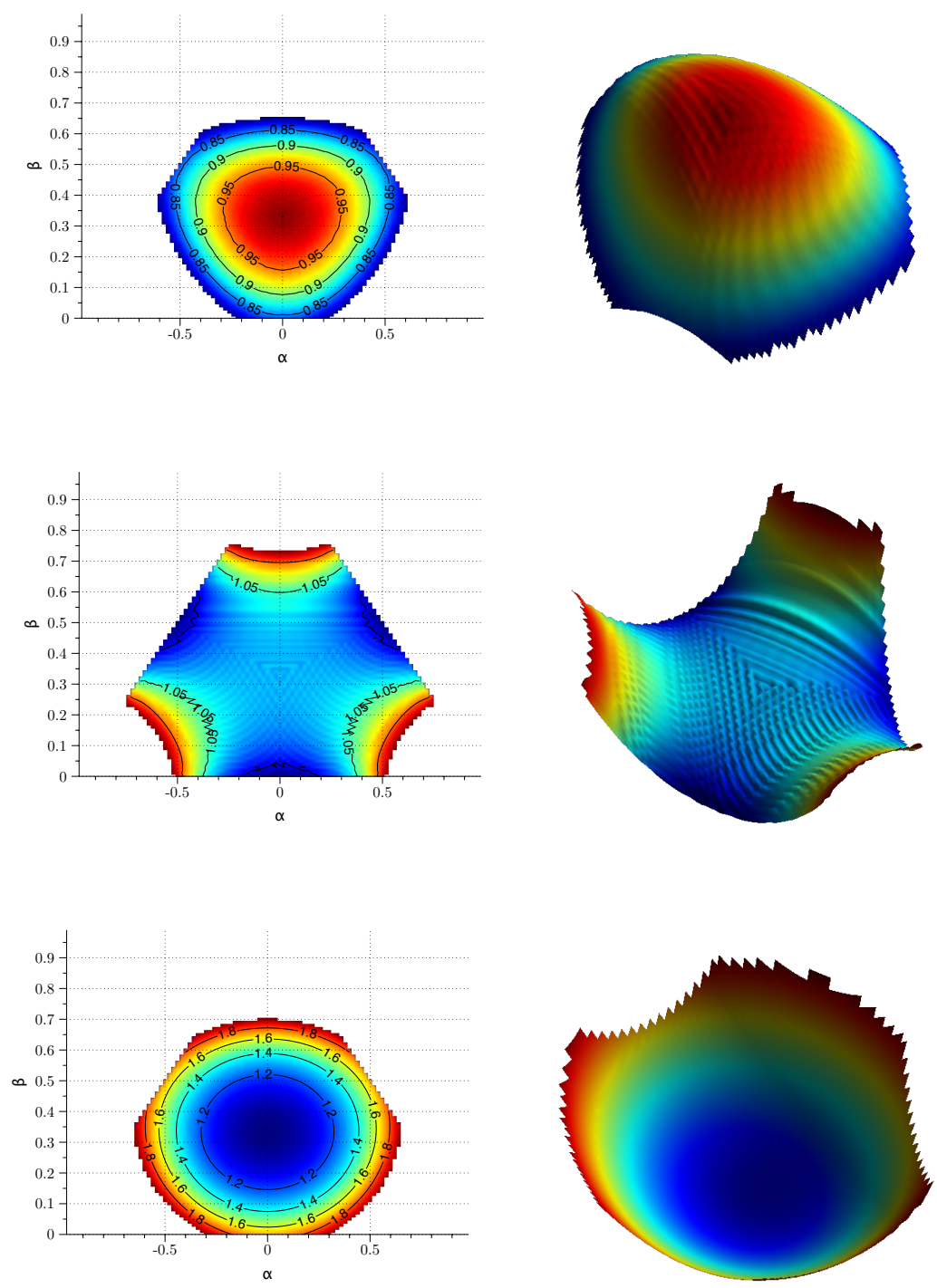

Figure 6. Zoom into the central regions of the dimensionless bispectrum $\mathcal{B}(\alpha, \beta)$ in the axion-quartic model (9.5). Regions near the vertices of the triangle are masked away, removing the strong spikes near squeezed configurations.

be made more accurate by the inclusion of next-order terms.

In practice we have found these oscillations around components with small amplitude to be largely harmless; they damp out as phase oscillations decay near horizon exit and do not disrupt the superhorizon epoch. Of more concern is the appearance of noise or uncontrolled oscillations around an exponentially decaying profile such as those appearing in the autocorrelation functions. A feature of this type is likely to be symptomatic of numerical errors that will produce an inaccurate result at late times. The normal response should be to tighten the numerical tolerances, increase the number of e-folds of massless evolution (see $\S 10.1$ ), or both. 

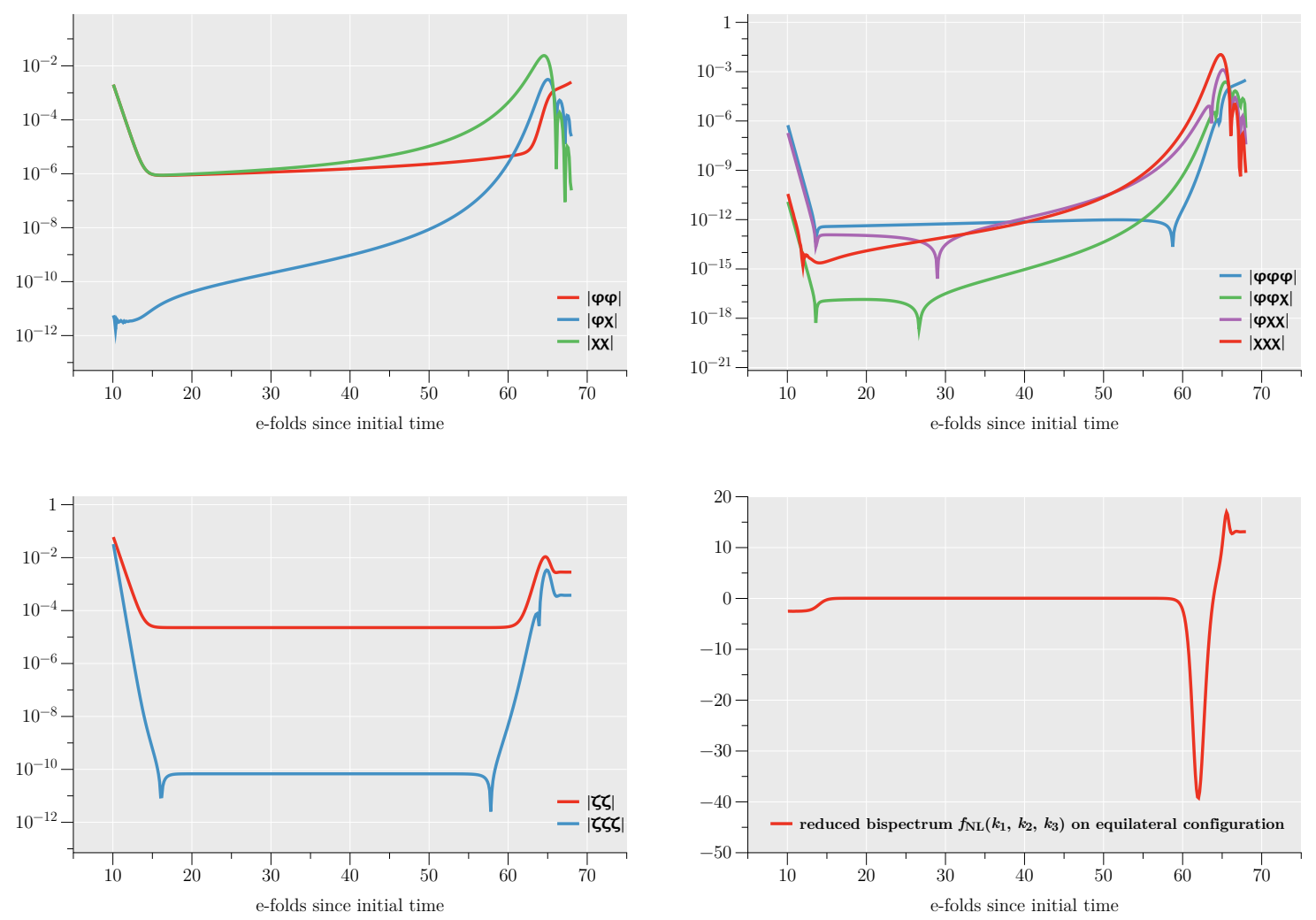

Figure 7. Time evolution of correlation functions for an equilateral configuration. Top left panel: field-space two-point functions. Top right panel: field-space three-point functions. Bottom left panel: $\zeta$ two- and three-point functions. Bottom right panel: reduced bispectrum. All plots except the bottom right panel show absolute values.

\subsection{Single-field model with feature}

The axion-quartic model yields a significant bispectrum from smooth field-space evolution. An alternative is to introduce a sharp 'feature' into the inflationary potential. In this section we show that our method handles such scenarios equally well. As an example, consider the single-field step model

$$
V(\phi)=\frac{1}{2} m^{2} \phi^{2}\left(1+c \tanh \frac{\phi-\phi_{0}}{d}\right) .
$$

This model was studied numerically by Chen, Easther \& Lim [46, 47], who computed both the two- and three-point functions. The step occurs at $\phi=\phi_{0}$. We take $m=10^{-5} M_{\mathrm{P}}$, $\phi_{0}=14.84 M_{\mathrm{P}}, c=0.0018$ and $d=0.022 M_{\mathrm{P}}$. The initial condition is $\phi=16.5 M_{\mathrm{P}}$, and its velocity is estimated using the slow-roll approximation.

The dimensionless power spectrum and reduced bispectrum on equilateral configurations are shown in Fig. 8. These are in good agreement with the results of Chen et al. [47]. In Fig. 9 we plot the shape of the dimensionless bispectrum $\mathcal{B}\left(k_{1}, k_{2}, k_{3}\right)$ and reduced bispectrum $f_{\mathrm{NL}}\left(k_{1}, k_{2}, k_{3}\right)$ at a fixed scale $k_{t}$, chosen so that $k_{t} / 3$ exits at $N=14.8$ e-folds from the initial time, corresponding to the peak of the largest positive spike of $f_{\mathrm{NL}}$ in Fig. 8. The 

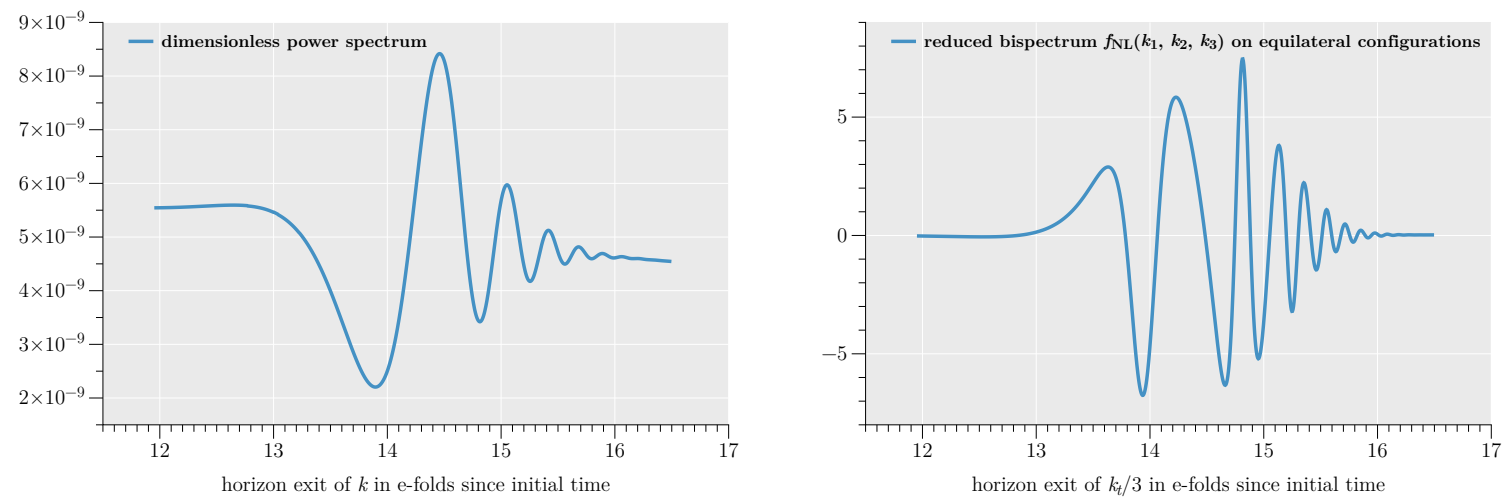

Figure 8. Left panel: dimensionless power spectrum in single-field feature model (9.7). Right panel: reduced bispectrum $f_{\mathrm{NL}}\left(k_{1}, k_{2}, k_{3}\right)$ on equilateral configurations as a function of scale $k_{t}$.
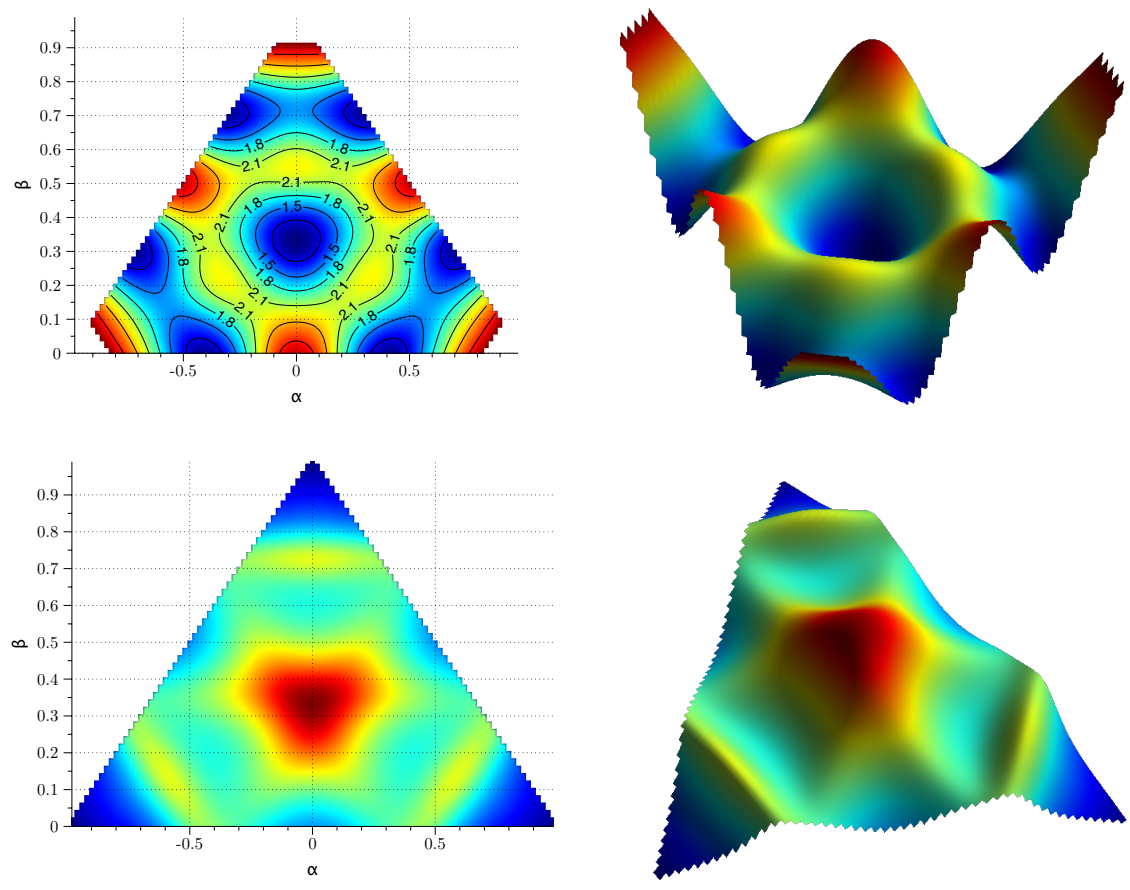

Figure 9. Top row: dimensionless bispectrum $\mathcal{B}(\alpha, \beta)$ as a function of the shape parameters $\alpha, \beta$. The squeezed regions near the vertices of the triangle have been removed. Bottom row: reduced bispectrum, also as a function of shape.

conclusion is that not only the scale dependence but also the shape dependence is very significant. Consequently, making a prediction for late-time observables that are sensitive to the bispectrum is a complex question. Accurate predictions are likely to require a detailed numerical study. 


\subsection{Heavy modes: adiabatic and non-adiabatic effects}

The discussion in \$§9.1-9.2 established that our numerical method can successfully track very delicate features in the shape- and scale-dependence of the bispectrum. We now study examples that illustrate the effect of heavy modes as described in $\S 2$ and demonstrate that we can successfully predict their $n$-point functions.

\subsubsection{Adiabatic-like models: gelaton and QSFI}

First consider the gelaton and QSFI scenarios. These are 'adiabatic-like' scenarios in which the expectation value of the heavy field tracks the minimum of its effective potential. As we now explain, such models appear to be rare if we attempt to engineer them from a Cartesian field-space metric and a turning trajectory. In fact, we have not yet managed to construct an explicit model that yields a significant bispectrum from either of these effects.

Consider a model with canonically-normalized Cartesian fields $X$ and $Y$, and translate to polar coordinates $X=R \cos \theta, Y=R \sin \theta$. The action for $R$ and $\theta$ becomes

$$
S=-\frac{1}{2} \int \mathrm{d}^{4} x \sqrt{-g}\left[(\partial R)^{2}+R^{2}(\partial \theta)^{2}+2 V(R \cos \theta, R \sin \theta)\right]
$$

where $V(X, Y)$ is the original potential. To study heavy-field effects we should choose the radial direction $R$ to be heavy and the angular direction $\theta$ to be light. The effective metric experienced by $\theta$ is non-Euclidean. This idea was suggested by Chen \& Wang [62]; see also Assassi et al. [112].

We can assume that $R$ is stabilized at some radius $R_{0}$. If the motion is purely rotational with angular velocity $\omega=\dot{\theta} / H=\mathrm{d} \theta / \mathrm{d} N$ then the slow-roll parameter $\epsilon$ satisfies

$$
\epsilon \approx \frac{R_{0}^{2}}{2 M_{\mathrm{P}}^{2}} \omega^{2}
$$

The adiabatic power spectrum $\mathcal{P}$ will be

$$
\mathcal{P} \sim \frac{H^{2}}{\epsilon M_{\mathrm{P}}^{2}} \sim \frac{H^{2}}{R_{0}^{2}} \frac{1}{\omega^{2}}
$$

To achieve a suitable normalization requires $R_{0}$ to be much larger than $H$, roughly $R_{0} \sim$ $H / \omega \mathcal{P}^{1 / 2}$. Successful inflation requires $\epsilon<1$ and therefore $H \lesssim M_{\mathrm{P}} \mathcal{P}^{1 / 2}$.

Gelaton scenario. - In a gelaton scenario the radial mass should be some scale $M$ at least modestly larger than $H$. We set $V^{\prime \prime}\left(R_{0}\right)=M^{2} \gtrsim H^{2}$, and take all higher derivatives to be negligible. For a circular trajectory we expect a significant gelaton-like renormalization of the sound speed when $\omega \gg M / H$ [65]. Such a large angular velocity implies that a full $2 \pi$-rotation in field space occurs in substantially less than an e-fold.

As a consequence of this constraint we have been unable to find a parameter combination that gives a significant gelaton effect over a meaningful range of scales. The conclusion 
appears to be that prolonged gelaton-like behaviour requires a more non-canonical metric than can be produced by angular rotation in the $X, Y$ model. ${ }^{14}$

QSFI scenario.- - Now consider a QSFI-like scenario. In this case the radial mass and radial cubic coupling should both be close to $H$, and the angular velocity $\omega$ should be a little smaller than unity in order to give efficient transfer of the radial fluctuations into the adiabatic mode [63]. Therefore there is no longer a problem associated with excessively rapid rotation; for example, we could pick $\omega \approx \pi / 30 \approx 0.1$. This would allow $N \approx 30$ e-folds for a rotation of $\pi$ around the origin.

The centrifugal force associated with angular motion will cause the $R$ expectation value to be displaced from its bare minimum $R_{0}$ by an amount $\Delta$. The effective potential experienced by radial fluctuations $\delta R$ around this displaced expectation value is

$$
\begin{aligned}
V_{\mathrm{eff}}(\delta R) & \approx \frac{1}{2}\left[V^{\prime \prime}\left(R_{0}\right)+V^{\prime \prime \prime}\left(R_{0}\right) \Delta+\frac{1}{2} V^{\prime \prime \prime \prime}\left(R_{0}\right) \Delta^{2}\right] \delta R^{2}+\frac{1}{3 !}\left[V^{\prime \prime \prime}\left(R_{0}\right)+V^{\prime \prime \prime \prime}\left(R_{0}\right) \Delta\right] \delta R^{3}+\cdots, \\
& =\frac{1}{2} \mu^{2} \delta R^{2}+\frac{1}{3 !} g \delta R^{3}
\end{aligned}
$$

where a prime' denotes a derivative with respect to $R$, and we have introduced the effective mass-squared $\mu^{2}$ and cubic coupling $g$. In each term we have kept derivatives up to $V^{\prime \prime \prime \prime}\left(R_{0}\right)$.

The presence of a large scale $R_{0} \gg H$ makes it difficult to keep the dressed mass $\mu$ smaller than $H$. To illustrate the problem, assume that the bare mass-squared $V^{\prime \prime}\left(R_{0}\right)$ is of order $H^{2}$ or smaller. Adopting a larger bare value can only make tuning problems worse.

First assume that $V^{\prime \prime \prime \prime}\left(R_{0}\right)$ is negligible. The cubic derivative $V^{\prime \prime \prime}\left(R_{0}\right)$ will control the displacement $\Delta$ when $\left|V^{\prime \prime \prime}\left(R_{0}\right)\right| \gtrsim \mathcal{P}^{1 / 2} H / \omega$, which will always be true if we choose $V^{\prime \prime \prime}\left(R_{0}\right) \sim H$ in order to achieve a QSFI phenomenology. The displacement is then

$$
\Delta \approx\left(\frac{2 H^{2}}{V^{\prime \prime \prime}\left(R_{0}\right) R_{0}}\right)^{1 / 2} \omega R_{0} .
$$

This makes a contribution to the mass-squared of order

$$
\delta \mu^{2} \supseteq V^{\prime \prime \prime}\left(R_{0}\right) \Delta \sim \omega^{1 / 2} \mathcal{P}^{-1 / 4} H^{3 / 2} \sqrt{V^{\prime \prime \prime}\left(R_{0}\right)} \gg H^{2} .
$$

Unless it is cancelled, this large contribution to the mass causes radial fluctuations to decay rapidly. It suppresses the transfer of any isocurvature bispectrum into the adiabatic mode $[64] .{ }^{15}$

\footnotetext{
${ }^{14}$ As an alternative one could consider a spiral-like model, which might accommodate a large $\omega$ by allowing multiple windings around the origin. Unfortunately it is still not easy to construct an explicit model. As we will discuss below, centrifugal forces associated with rotation will cause the $R$ expectation value to be displaced away from its bare minimum $R_{0}$ by an amount $\Delta$. Assuming this displacement to be controlled by the quadratic term, choosing $\omega \sim M / H$ gives $\Delta \sim R_{0}$. Therefore no more than a few spirals can be packed into the region $0 \leqslant R \leqslant R_{0}$.

${ }^{15}$ As explained in $\S 3.2 .2$, this conversion is captured by a tree-level calculation because it occurs via quadratic mixing rather than decay of two or more particles. Therefore we expect our tree-level codes to fully support the QSFI phenomenology.
} 

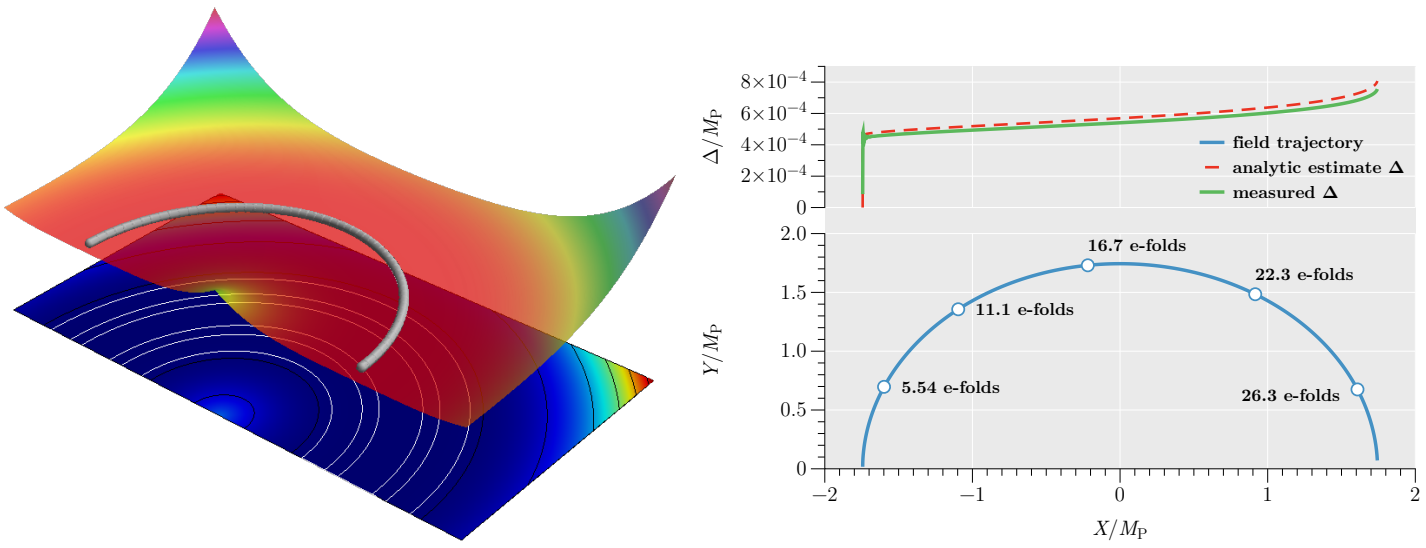

Figure 10. Potential and trajectory for the model (9.14). Left: Potential and trajectory. The fields roll from the far to the near side, and the lower plane shows equipotential contours. Right: zoomed plot of the trajectory, showing the displacement $\Delta$.
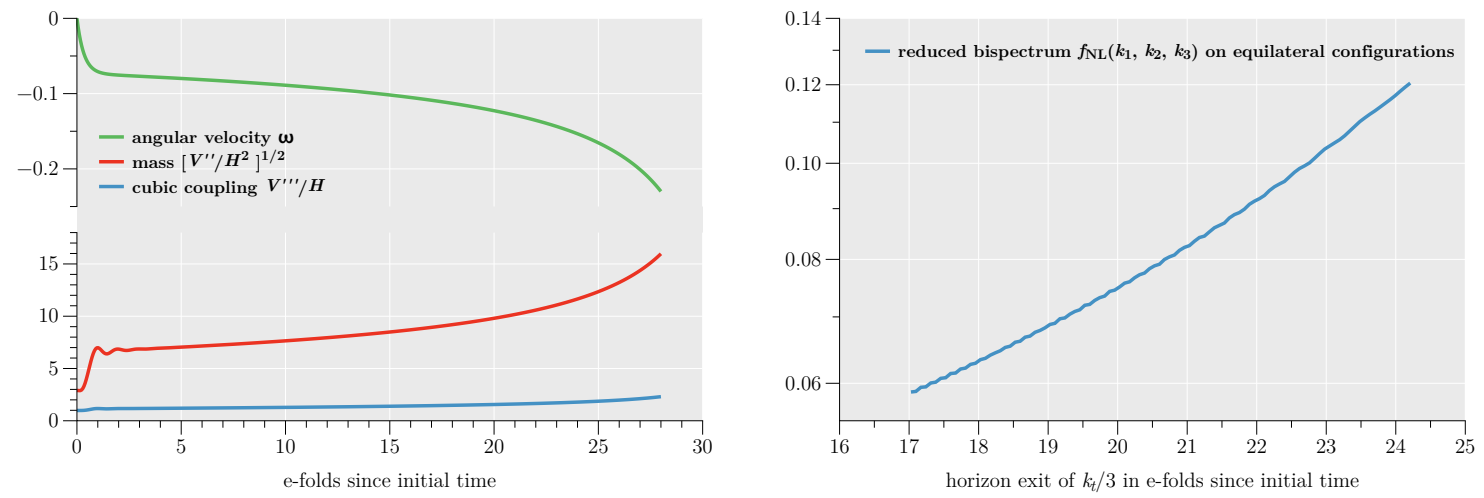

Figure 11. Left: evolution of angular velocity $\omega=\dot{\theta} / H$, together with the mass $\mu / H$ and coupling $g / H$. Right panel: reduced bispectrum evaluated on equilateral configurations as a function of scale $k_{t}$.

Similar estimates can be made for more complex parameter combinations, but in the cases we have checked there is always a contribution to $\delta \mu^{2}$ whose scale is set by $H^{2}$. The possibility of constructing a model in which the radial mass is $<H^{2}$ then rests on the precise $\mathrm{O}(1)$ factors that occur. A representative example is

$$
V=V_{0}\left(1+\frac{29 \pi}{120} \theta+\frac{1}{2} \frac{\eta_{R}}{M_{\mathrm{P}}^{2}}\left(R-R_{0}\right)^{2}+\frac{1}{3 !} \frac{g_{R}}{M_{\mathrm{P}}^{3}}\left(R-R_{0}\right)^{3}+\frac{1}{4 !} \frac{\lambda_{R}}{M_{\mathrm{P}}^{4}}\left(R-R_{0}\right)^{4}\right),
$$

with the parameters $V_{0}=10^{-10} M_{\mathrm{P}}^{4}, \eta_{R}=1 / \sqrt{3}, g_{R}=M_{\mathrm{P}}^{2} V_{0}^{-1 / 2}$ and $\lambda_{R}=0.5 M_{\mathrm{P}}^{3} \omega^{-1 / 2} V_{0}^{-3 / 4}$. We plot the potential and inflationary trajectory in Figure 10.

In Fig. 11 we show the time evolution of the angular velocity $\omega=\dot{\theta} / H$ together with the dressed mass $\mu / H$ and cubic coupling $g / H$. The mass lies roughly in the range $5 H$ to $15 H$ and the cubic coupling lies roughly between $H$ and $2 H$. In the right panel we show the 
reduced bispectrum produced by this model on equilateral configurations. The amplitude is of order $10^{-1}$. It would be interesting to search for a parameter combination (even if very finely tuned) that yields a more significant bispectrum, but we leave this issue for future work.

Although we have not successfully reproduced a QSFI effect with significant amplitude, this model demonstrates the ability of our numerical tools to handle scenarios with Hubblescale masses or sizeable cubic couplings.

\subsubsection{Non-adiabatic model: particle production}

It is much easier to construct models that realize nonadiabatic effects. In this section we study a model introduced by Gao, Langlois \& Mizuno [70], which is designed so that the inflationary trajectory contains a turn through an angle $\Delta \theta$. If the turn is sufficiently sharp then it will force the heavy field away from its minimum, and the subsequent relaxation will be nonadiabatic.

The potential used by Gao et al. was

$$
V(X, Y)=\frac{1}{2} m_{X}^{2} X^{2}+\frac{1}{2} M^{2} \cos ^{2} \frac{\Delta \theta}{2}\left[Y-\left(X-X_{0}\right) \tan \Xi(X)\right]^{2},
$$

where $\Xi(X)$ is defined by

$$
\Xi(X)=\frac{\Delta \theta}{\pi} \arctan s\left(X-X_{0}\right) .
$$

The parameter $s$ controls the sharpness of the turn, which occurs near $(X, Y)=\left(X_{0}, 0\right)$. Larger values of $s$ generate a sharper turn. We take $M=10^{-4} M_{\mathrm{P}}, m_{X}=10^{-7} M_{\mathrm{P}}$, and $\Delta \theta=\pi / 10$. The turn is positioned at $X_{0}=(231-100 \sqrt{6}) M_{\mathrm{P}} \approx-14 M_{\mathrm{P}}$ and the sharpness parameter is $s=1000 \sqrt{3} / M_{\mathrm{P}} \approx 1700 / M_{\mathrm{P}}$. We choose initial conditions $X=(229-100 \sqrt{6}) M_{\mathrm{P}} \approx-16 M_{\mathrm{P}}$ and $Y=2 M_{\mathrm{P}} \tan (\pi / 20)$. The potential and trajectory are shown in the left-hand panel of Fig. 12. The right-hand panel shows the trajectory in more detail, including a zoomed region focused on the turn. When viewed as a whole the trajectory has the appearance of two straight lines joining abruptly at $N \approx 16$ e-folds from the initial time. With sufficient magnification, however, the small oscillations characterizing nonadiabatic relaxation are clearly visible. The changing mass associated with these oscillations leads to stimulated particle production [79].

We plot the dimensionless power spectrum and reduced bispectrum on equilateral configurations in Fig. 13. The power spectrum shows a significant enhancement of power associated with the turn, with the enhancement peaking for scales that exit the horizon just after $N=16$ e-folds from the initial time. Smaller scales show decaying oscillations, eventually returning to the continuum level when the non-adiabatic evolution has died away. In the power spectrum these oscillations become negligible for scales that exit the horizon $N \gtrsim 21$ e-folds from the initial time.

The reduced bispectrum also shows significant enhancement, but with a more complex structure. There is some growth around the turn at 16 e-folds, but the most dramatic effects occur later during the oscillating phase. There are rapid, large-amplitude oscillations achieving up to $\mid f_{\mathrm{NL}}\left(k_{1}, k_{2}, k_{3} \mid \sim 600\right.$. As the heavy field returns to adiabatic evolution these 

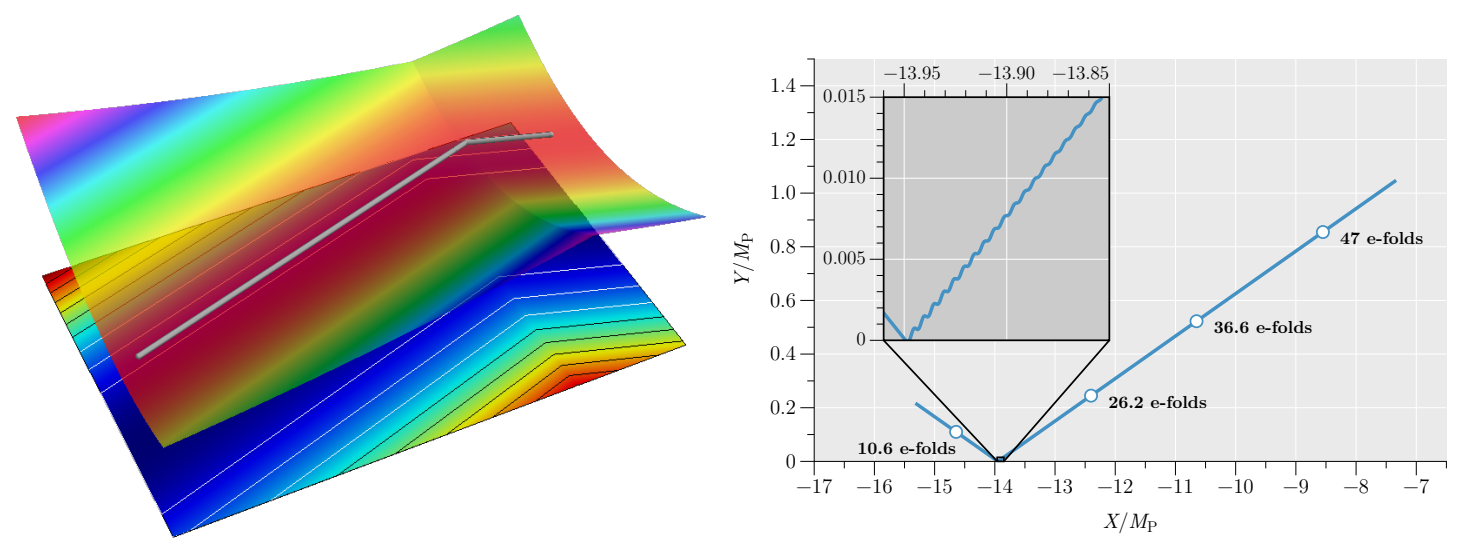

Figure 12. Potential and trajectory for the nonadiabatic model (9.15). Left: Potential and trajectory. The fields roll from the far to the near side, and the lower plane shows equipotential contours. Right: zoomed plot of the trajectory.
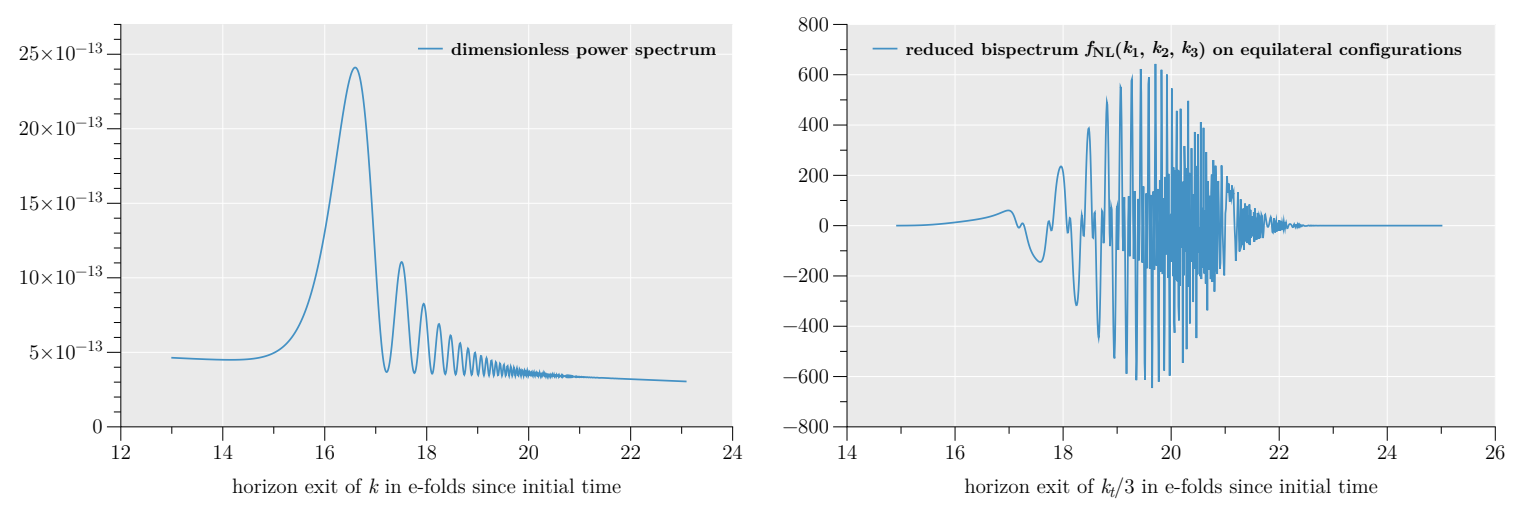

Figure 13. Left panel: dimensionless power spectrum produced in the nonadiabatic model (9.15). Right panel: reduced bispectrum evaluated on equilateral configurations as a function of scale $k_{t}$.

oscillations decrease in amplitude, and the bispectrum returns to its background level. The transition occurs on slightly smaller scales than for the power spectrum, for scales $k_{t}$ that exit the horizon $N \gtrsim 23$ e-folds from the initial time.

Comparison with analytic estimates.-Gao, Langlois \& Mizuno developed an analytic framework in which to estimate the power spectrum produced by (9.15), but did not discuss the bispectrum [70,71]. Achúcarro and collaborators introduced a similar framework and used it to study contributions to both the power spectrum and bispectrum, but their analysis assumed that any features were only moderately sharp [68, 69]. Chen et al. studied single-field models with sharp features [47]. Adshead et al. later developed an alternative single-field framework based on a Green's function solution to the mode equations [49]. More recently, Flauger et al. estimated the bispectrum from particle production in two field model which exhibits an approximate discrete shift symmetry [80]. 
None of these formalisms are exactly applicable to (9.15) with our parameter choices. However, the general structure visible in Fig. 13- a sudden increase in the two-point function, followed by decaying oscillations, and rapid oscillations embedded within a 'pulse' of large amplitude in the three-point function - are qualitatively in very good agreement with Refs. [49, 68, 69].

\section{Performance and scaling behaviour}

Before concluding, we pause to discuss the performance of our method - and especially how the integration time scales with different choices for key parameters such as the number of e-folds of massless subhorizon evolution. To illustrate these properties we use the model of double quadratic inflation,

$$
V=\frac{1}{2} m_{\phi}^{2} \phi^{2}+\frac{1}{2} m_{\chi}^{2} \chi^{2}
$$

This potential was used by Rigopoulos, Shellard \& van Tent to provide a simple test-case for numerical methods [113, 114]; see also Refs. [18, 21, 59, 60, 115, 116]. Because the potential is sum-separable it falls into a small class where analytical results for the factorization coefficients $N_{a}$ and $N_{a b}$ can be computed using the slow-roll approximation; these calculations were performed by Vernizzi \& Wands [56]. In our computations we take $m_{\phi}=9 \times 10^{-5} M_{\mathrm{P}}$ and $m_{\chi}=1 \times 10^{-5} M_{\mathrm{P}}$. The initial conditions are $\phi=10 M_{\mathrm{P}}$ and $\chi=12.9 M_{\mathrm{P}}$, and their derivatives are set using the slow-roll approximation.

\subsection{Number of massless subhorizon e-folds}

It was explained in $\S 6$ that our strategy of computing initial conditions using the massless approximation forces us to position the initial time sufficiently early that $(k / a)^{2} \gg m^{2}$, where $m^{2}$ is the largest eigenvalue of the mass matrix. Typically $m^{2}$ will itself be time dependent. We define the massless time for the mode $k$ to occur when $(k / a)^{2}=m^{2}$, or at horizon crossing $(k / a)^{2}=H^{2}$, whichever is earlier. ${ }^{16}$

The initial time for a given wavenumber configuration should be placed at least a few e-folds earlier than the earliest massless time with which it is associated. (By an extension of terminology we say that this earliest massless time is the massless time for the configuration as a whole.) The earlier we take the initial time, the more accurate the calculation of initial conditions should be. Typically this means that numerical accuracy improves as we increase the number of e-folds spent in the massless phase. On the other hand, integration in this phase is expensive and we must often make a choice that balances runtime against accuracy.

The CppTransport platform allows a user to automatically position the initial time for each configuration a fixed number of e-folds $N_{\text {pre }}$ prior to its massless time. The same choice can be made with PyTransport using a set of supplied scripts. In this subsection we study how runtime and accuracy vary as we adjust $N_{\text {pre }}$.

Convergence.- - In Fig. 14 we plot the final value of the dimensionless $\zeta$ bispectrum $\mathcal{B}\left(k_{1}, k_{2}, k_{3}\right)$ for four wavenumber configurations:

\footnotetext{
${ }^{16}$ This terminology is a convenient shorthand, but one should not be misled. When $(k / a)^{2}=m^{2}$ the kinetic energy strictly balances the potential energy from the mass. To be in the massless regime we need $(k / a)^{2} \gg m^{2}$.
} 

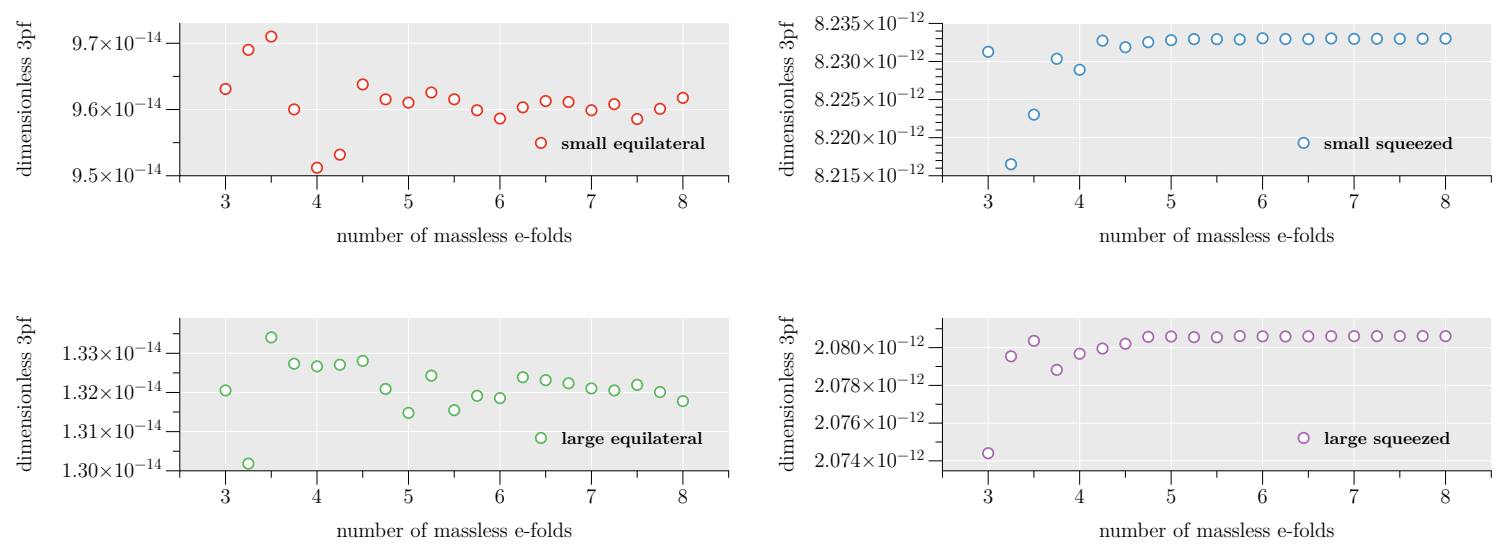

Figure 14. Convergence properties of the numerical solution with increasing number of massless subhorizon e-folds.

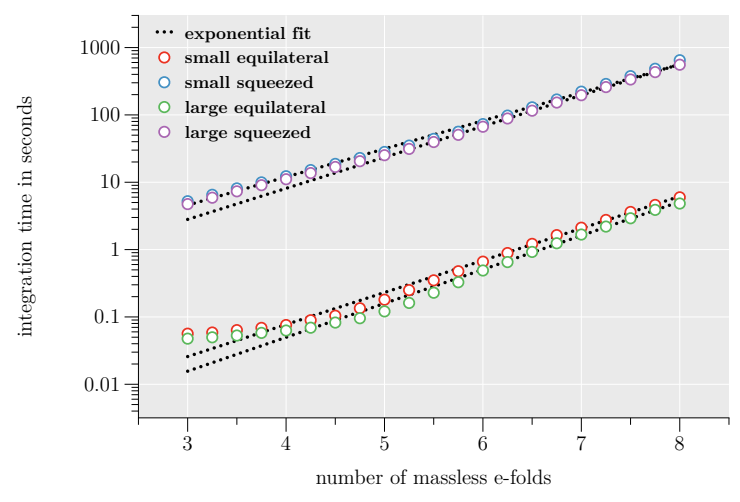

Figure 15. Scaling of integration time with increasing number of massless (or subhorizon) e-folds.

1. Top left: a 'small' equilateral configuration for which $k_{t} / 3$ has horizon-exit time $\approx 19.0$ e-folds after the initial conditions.

2. Top right: a 'small' squeezed configuration with the same $k_{t}$ as above, but $\alpha=0$ and $\beta=0.99$.

3. Bottom left: a 'large' equilateral configuration for which $k_{t} / 3$ has horizon-exit time $\approx 24.5$ e-folds after the initial conditions.

4. Bottom right: a 'large' squeezed configuration with the same value for $k_{t}$, but $\alpha=0$ and $\beta=0.99$.

It is clear that the results are very stable, especially for the squeezed configurations. The equilateral configurations shows some scatter but the numerical value settles down for $N_{\text {pre }} \gtrsim$ 4.5 . 
Scaling of integration time with $N_{\text {pre }}$ - - In Fig. 15 we plot the corresponding integration times $T$ required by the CppTransport platform, together with dotted lines showing fits to the scaling law $T \propto \mathrm{e}^{\alpha N_{\text {pre }}} .{ }^{17}$ The squeezed configurations fall nearly exactly on such a law with $\alpha \approx 1$, and the same is true for the equilateral configuration at sufficiently large $N_{\text {pre }}$. The same scaling is seen with PyTransport.

The conclusion is that increasing the number of massless e-folds is exponentially expensive. The reasons for this behaviour were explained in $\$ 9.1$ above, and can ultimately be traced to increasingly rapid phase oscillations on subhorizon scales. Fortunately, Fig. 14 shows that convergence is fairly rapid, and beyond a certain point there are diminishing returns. Therefore very large values of $N_{\text {pre }}$ are seldom necessary; choices in the range 3 to 5 give reasonable results for typical models. In more complex cases where a dynamical feature occurs near horizon exit it is necessary to use larger $N_{\text {pre }}$, in some cases as large as 7 or 8 .

\subsection{Shape-dependence}

We have seen that the number of e-folds of massless evolution is a key factor in determining the integration time. A second factor is the shape of the wavenumber configuration, as measured by $\alpha$ and $\beta$. In particular, strongly squeezed configurations contain one wavenumber that is much smaller than the other two. Without loss of generality we can take this to be $k_{3}$, so we are focusing on configurations for which $k_{3} \ll k_{2} \sim k_{3} \sim k_{t} / 2$.

The massless time for this configuration will be determined by the massless time for $k_{3}$, which may be many e-folds earlier than the massless time for $k_{1} \sim k_{2}$. This means that there will be a long phase of exponential decay associated with terms of the form $k_{1} / a H$ and $k_{2} / a H$, and on the basis of what has been said in $\$ 10.1$ this phase will be expensive to integrate. Therefore we should expect the integration time to scale strongly with the 'squeezedness' of the configuration. Since we are taking $k_{3}$ to be the squeezed wavenumber this limit occurs when $\beta \uparrow 1$, but to measure the scaling it proves to be more convenient to work in terms of $k_{3} / k_{t}=(1-\beta) / 2$. In Fig. 16 we plot the integration time for each configuration used to construct the bispectrum shape plots in Figs. 5 and 6. Recall that these configurations have fixed $k_{t}$ but varying $\alpha$ and $\beta$.

In Fig. 16 the different $\alpha$ configurations are visible as a cluster of points near $k_{3} / k_{t} \sim \frac{1}{3}$. For $k_{3} / k_{t}<1$ the allowed triangular configurations become rarer, and eventually for very small $k_{3} / k_{t}$ there are only isosceles configurations with $\alpha=0$. In the squeezed region the timing data can be roughly fit by a power law $\left(k_{3} / k_{t}\right)^{-1.17}$.

The precise details of the scaling vary with the model under discussion, but the dependence on $k_{3} / k_{t}$ is normally a power-law. In some models there can be one or more breaks between different power laws, depending (among other possibilities) on the dynamical behaviour of the background. Broadly speaking we find the power-law index lies between -1 and -2 . For example, for the complex scaling model discussed in $\S 4$ of Byrnes et al. [74], we find that the time required in the deeply squeezed limit scales like $\left(k_{3} / k_{t}\right)^{-1.64}$. We conclude that strongly squeezed configurations are expensive to compute. This is unfortunate

\footnotetext{
${ }^{17}$ The test configuration was an iMac13,2: $3.4 \mathrm{GHz}$ Intel Core i7-3770 (Ivy Bridge), macOS 10.11.5, clang703.0.29, Boost 1.59.
} 


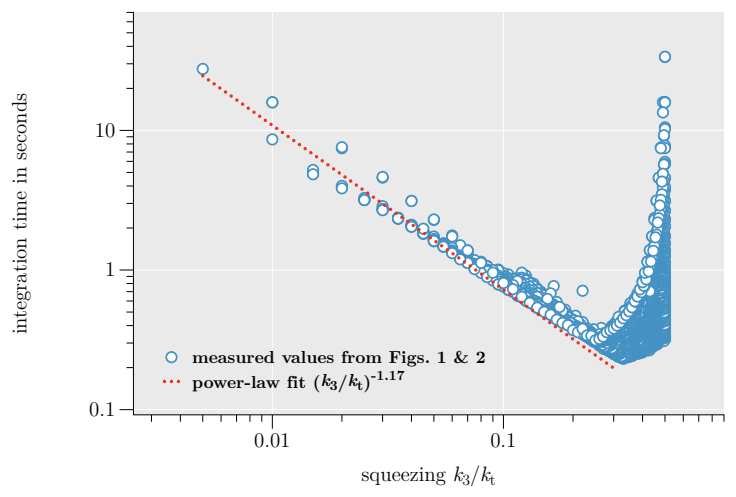

Figure 16. Dependence of integration time on the squeezing parameter $k_{3} / k_{t}$.

given that some observables are principally sensitive to these configurations, such as the scale-dependent galaxy bias or the position-dependent power spectrum.

\section{Conclusions}

The major result of this paper is a complete formalism for numerical calculation of the tree-level correlation functions produced during an epoch of early-universe inflation. This formalism was described in $\S \S 3-7$. It does not require the slow-roll approximation except to obtain the estimates of initial conditions given in $\S 6$. As explained in $\S 3.2$, the tree-level approximation means that our formalism should produce accurate estimates unless multiparticle production channels make a significant contribution to the curvature perturbation $\zeta$, for example by nontrivial scattering processes or decays. In certain scenarios, such as warm inflation or trapped inflation, the tree approximation may also fail to capture processes by which energy is drained from the zero-mode into finite-wavenumber excitations. These restrictions should be carefully considered before applying our tools - or any others based on a tree-level approximation - to study some particular inflationary model.

We are supplying two concrete implementations of this formalism, described in $\S 8$ and available for download under open source licences. These are not just bare implementations of the evolution equations; instead, they both support automated analysis of models from a high-level Lagrangian description, and can be used to produce immediate high-resolution numerical results for models whose bispectra were previously intractable.

In $\S \S 3-7$ we focused on the correlation functions generated by a system of canonicallynormalized scalar fields (and their contribution to the curvature perturbation $\zeta$ ), and the two bispectrum-level implementations described in $\S 8$ are currently restricted to scenarios of this type. However, this restriction is not necessary as a matter of principle. Extensions to more complex models, such as those with a nontrivial kinetic sector $G_{\alpha \beta}(\phi) \partial_{a} \phi^{\alpha} \partial^{a} \phi^{\beta}$ or interactions of Galileon-type, are limited only by algebraic complexity. All that would be required are replacements for the tensors $u_{\mathrm{b}}^{\mathrm{a}}$ and $u_{\mathrm{bc}}^{\mathrm{a}}$, and appropriate initial conditions that account for the new interactions and kinetic structure. An older Mathematica-based implementation 
capable of handling models with nontrivial field-space metric is already available, although its output is limited to the two-point function [24]. All three implementations, together with links to further resources, can be found at the website transportmethod.com.

In $\S 9$ we exhibited results for a selection of concrete models exemplifying the wide range of mass spectra and coupling constants that can be accommodated. Collectively, these demonstrate that our formalism successfully tracks highly nuanced features of the bispectrum amplitude and shape. Just as important, because it includes all relevant effects (especially from gravitational-strength couplings), it can be used to predict the complete scale- and shape-dependent bispectrum generated in models where the reduced bispectrum $f_{\mathrm{NL}}\left(k_{1}, k_{2}, k_{3}\right)$ is order unity. These models are a target for the next generation of galaxy surveys, including Euclid, DESI and LSST [117]. To obtain robust predictions with these low amplitudes it is not sufficient to rely on approximations that discard physical effects occurring on subhorizon scales or around horizon exit, or that do not accurately account for the effect of hierarchies among the wavenumbers appearing in each correlation function. Our formalism, and especially the reusable implementations we provide, supply a means for these models to be accurately analysed for the first time.

Meanwhile, to achieve a suitable level of preparation for a Euclid-, DESI- or LSST-like survey it will be insufficient merely to improve the accuracy of primordial calculations. Reliable forecasts for models where $\left|f_{\mathrm{NL}}\left(k_{1}, k_{2}, k_{3}\right)\right| \lesssim 1$ must at least account for gravitational evolution after horizon exit and the characteristics of the survey. These details are now wellunderstood; what is required is an integrated toolchain that links them all together. At the simpler level required by CMB experiments, an analysis such as that given in Ref. [74] — which relied on numerical bispectra produced using the CppTransport platform - demonstrates how accurate, high-resolution calculations of primordial correlation functions can be integrated into a numerical toolchain producing accurate, reliable results customized to a specific experiment. (In Ref. [74] the customization was for a Planck-like CMB survey, but the point we are making is much more general.) As we stockpile datasets of ever-increasing accuracy there is a corresponding burden on theorists to generate predictions with matching refinement. No one tool, or single approach, will be sufficient-but we hope that that the software tools we are making available constitute a step towards this goal.

\section{Acknowledgments}

PyTransport.-Development of PyTransport has been supported by the Royal Society through the support given to DJM by a Royal Society University Research Fellowship and by Science and Technology Facilities Council grant ST/J001546/1.

CppTransport.-Development of CppTransport has been supported significantly by an ERC grant:

- Precision tests of the inflationary scenario, funded by the European Research Council under the European Union's Seventh Framework Programme (FP/2007-2013) and ERC Grant Agreement No. 308082. (MD, DS) 
In addition, some development of CppTransport has been supported by other funding sources. Portions of the work described in this document have been supported by:

- The UK Science and Technology Facilities Council via grants ST/I000976/1 and ST/L000652/1, which funded the science programme at the University of Sussex Astronomy Centre from April 2011-March 2014 and April 2014-March 2017, respectively. (MD, JF, DS)

- The Leverhulme Trust via a Philip Leverhulme Prize. (DS)

- The National Science Foundation Grant No. PHYS-1066293 and the hospitality of the Aspen Center for Physics. (MD, JF, DS)

- The hospitality of the Higgs Centre for Theoretical Physics at the University of Edinburgh, and the Centre for Astronomy \& Particle Physics at the University of Nottingham. (DS)

In addition, MD acknowledges funding from the German Science Foundation (DFG) within the Collaborative Research Centre 676 Particles, Strings and the Early Universe and by the ERC Consolidator Grant STRINGFLATION under the HORIZON 2020 contract no. 647995, and JF acknowledges funding from the ERC Consolidator Grant STRINGFLATION under the HORIZON 2020 contract no. 647995 and a grant from the Simons Foundation.

Data availability statement.- No new data were collected for the preparation of this paper. All plots and figures are generated by computer codes. These codes are freely available for download as described in $\S 8$.

\section{References}

[1] C. Degrande, C. Duhr, B. Fuks, D. Grellscheid, O. Mattelaer and T. Reiter, UFO - The Universal FeynRules Output, Comput. Phys. Commun. 183 (2012) 1201-1214, [1108.2040].

[2] A. Semenov, LanHEP: A package for automatic generation of Feynman rules from the Lagrangian, Comput. Phys. Commun. 115 (1998) 124-139.

[3] A. Semenov, LanHEP: A Package for the automatic generation of Feynman rules in field theory. Version 3.0, Comput. Phys. Commun. 180 (2009) 431-454, [0805. 0555].

[4] N. D. Christensen and C. Duhr, FeynRules - Feynman rules made easy, Comput. Phys. Commun. 180 (2009) 1614-1641, [0806.4194].

[5] N. D. Christensen, P. de Aquino, C. Degrande, C. Duhr, B. Fuks, M. Herquet et al., A Comprehensive approach to new physics simulations, Eur. Phys. J. C71 (2011) 1541, [0906.2474].

[6] CompHEP collaboration, E. Boos, V. Bunichev, M. Dubinin, L. Dudko, V. Ilyin, A. Kryukov et al., CompHEP 4.4: Automatic computations from Lagrangians to events, Nucl. Instrum. Meth. A534 (2004) 250-259, [hep-ph/0403113].

[7] A. Pukhov, E. Boos, M. Dubinin, V. Edneral, V. Ilyin, D. Kovalenko et al., CompHEP: A Package for evaluation of Feynman diagrams and integration over multiparticle phase space, hep-ph/9908288. 
[8] A. Pukhov, CalcHEP 2.3: MSSM, structure functions, event generation, batchs, and generation of matrix elements for other packages, hep-ph/0412191.

[9] J. Alwall, R. Frederix, S. Frixione, V. Hirschi, F. Maltoni, O. Mattelaer et al., The automated computation of tree-level and next-to-leading order differential cross sections, and their matching to parton shower simulations, JHEP 07 (2014) 079, [1405.0301].

[10] T. Hahn and M. Perez-Victoria, Automatized one loop calculations in four-dimensions and D-dimensions, Comput. Phys. Commun. 118 (1999) 153-165, [hep-ph/9807565].

[11] T. Hahn and M. Rauch, News from FormCalc and LoopTools, Nucl. Phys. Proc. Suppl. 157 (2006) 236-240, [hep-ph/0601248].

[12] C. Ringeval, P. Brax, C. van de Bruck and A.-C. Davis, Boundary inflation and the WMAP data, Phys.Rev. D73 (2006) 064035, [astro-ph/0509727].

[13] J. Martin and C. Ringeval, Inflation after WMAP3: Confronting the Slow-Roll and Exact Power Spectra to CMB Data, JCAP 0608 (2006) 009, [astro-ph/0605367].

[14] C. Ringeval, The exact numerical treatment of inflationary models, Lect.Notes Phys. 738 (2008) 243-273, [astro-ph/0703486].

[15] M. J. Mortonson, H. V. Peiris and R. Easther, Bayesian Analysis of Inflation: Parameter Estimation for Single Field Models, Phys.Rev. D83 (2011) 043505, [1007.4205].

[16] R. Easther and H. V. Peiris, Bayesian Analysis of Inflation II: Model Selection and Constraints on Reheating, Phys.Rev. D85 (2012) 103533, [1112.0326].

[17] J. Norena, C. Wagner, L. Verde, H. V. Peiris and R. Easther, Bayesian Analysis of Inflation III: Slow Roll Reconstruction Using Model Selection, Phys.Rev. D86 (2012) 023505, [1202.0304].

[18] L. C. Price, J. Frazer, J. Xu, H. V. Peiris and R. Easther, MultiModeCode: An efficient numerical solver for multifield inflation, JCAP 03 (2015) 005, [1410.0685].

[19] I. Huston and K. A. Malik, Numerical calculation of second order perturbations, JCAP 0909 (2009) 019, [0907.2917].

[20] I. Huston and K. A. Malik, Second Order Perturbations During Inflation Beyond Slow-roll, JCAP 1110 (2011) 029, [1103.0912].

[21] I. Huston and A. J. Christopherson, Calculating Non-adiabatic Pressure Perturbations during Multi-field Inflation, Phys.Rev. D85 (2012) 063507, [1111.6919].

[22] D. K. Hazra, L. Sriramkumar and J. Martin, BINGO: A code for the efficient computation of the scalar bi-spectrum, JCAP 1305 (2013) 026, [1201.0926].

[23] V. Sreenath, D. K. Hazra and L. Sriramkumar, On the scalar consistency relation away from slow roll, JCAP 1502 (2015) 029, [1410.0252].

[24] M. Dias, J. Frazer and D. Seery, Computing observables in curved multifield models of inflation - A guide (with code) to the transport method, JCAP 1512 (2015) 030, [1502.03125].

[25] C. Bauer and H. S. Do, One loop integrals with XLOOPS-GiNaC, Comput. Phys. Commun. 144 (2002) 154-168, [hep-ph/0102231].

[26] D. Seery, CppTransport: a platform to automate calculations of inflationary correlation functions, 2016. 10.5281/zenodo.61239.

[27] D. J. Mulryne, PyTransport: A fast python package for the calculation of inflationary 
correlation functions, 2016. 10.5281/zenodo.61265.

[28] D. Seery, A parton picture of de Sitter space during slow-roll inflation, JCAP 0905 (2009) 021, [0903.2788].

[29] N. Arkani-Hamed and J. Maldacena, Cosmological Collider Physics, 1503.08043.

[30] D. H. Lyth, Large Scale Energy Density Perturbations and Inflation, Phys.Rev. D31 (1985) 1792-1798.

[31] J. M. Bardeen, P. J. Steinhardt and M. S. Turner, Spontaneous Creation of Almost Scale-Free Density Perturbations in an Inflationary Universe, Phys.Rev. D28 (1983) 679.

[32] D. Wands, K. A. Malik, D. H. Lyth and A. R. Liddle, A new approach to the evolution of cosmological perturbations on large scales, Phys. Rev. D62 (2000) 043527, [astro-ph/0003278].

[33] D. H. Lyth and Y. Rodríguez, The inflationary prediction for primordial non-gaussianity, Phys.Rev.Lett. 95 (2005) 121302, [astro-ph/0504045].

[34] K. A. Malik, Gauge-invariant perturbations at second order: Multiple scalar fields on large scales, JCAP 0511 (2005) 005, [astro-ph/0506532].

[35] D. Seery, D. J. Mulryne, J. Frazer and R. H. Ribeiro, Inflationary perturbation theory is geometrical optics in phase space, JCAP 1209 (2012) 010, [1203.2635].

[36] G. Rigopoulos and E. Shellard, The separate universe approach and the evolution of nonlinear superhorizon cosmological perturbations, Phys.Rev. D68 (2003) 123518, [astro-ph/0306620].

[37] D. H. Lyth, K. A. Malik and M. Sasaki, A General proof of the conservation of the curvature perturbation, JCAP 0505 (2005) 004, [astro-ph/0411220].

[38] S. Weinberg, Non-Gaussian Correlations Outside the Horizon, Phys.Rev. D78 (2008) 123521, [0808.2909].

[39] S. Weinberg, Non-Gaussian Correlations Outside the Horizon II: The General Case, Phys.Rev. D79 (2009) 043504, [0810.2831].

[40] S. Weinberg, Quantum contributions to cosmological correlations, Phys. Rev. D72 (2005) 043514, [hep-th/0506236].

[41] S. Weinberg, Quantum contributions to cosmological correlations. II. Can these corrections become large?, Phys. Rev. D74 (2006) 023508, [hep-th/0605244].

[42] J. M. Maldacena, Non-Gaussian features of primordial fluctuations in single field inflationary models, JHEP 05 (2003) 013, [astro-ph/0210603].

[43] D. Seery and J. E. Lidsey, Primordial non-Gaussianities in single field inflation, JCAP 0506 (2005) 003, [astro-ph/0503692].

[44] X. Chen, M.-x. Huang, S. Kachru and G. Shiu, Observational signatures and non-Gaussianities of general single field inflation, JCAP 0701 (2007) 002, [hep-th/0605045].

[45] J. Elliston, D. Seery and R. Tavakol, The inflationary bispectrum with curved field-space, JCAP 1211 (2012) 060, [1208.6011].

[46] X. Chen, R. Easther and E. A. Lim, Large Non-Gaussianities in Single Field Inflation, JCAP 0706 (2007) 023, [astro-ph/0611645].

[47] X. Chen, R. Easther and E. A. Lim, Generation and Characterization of Large Non-Gaussianities in Single Field Inflation, JCAP 0804 (2008) 010, [0801.3295]. 
[48] H. Funakoshi and S. Renaux-Petel, A Modal Approach to the Numerical Calculation of Primordial non-Gaussianities, JCAP 1302 (2013) 002, [1211.3086].

[49] P. Adshead, W. Hu and V. Miranda, Bispectrum in Single-Field Inflation Beyond Slow-Roll, Phys. Rev. D88 (2013) 023507, [1303.7004].

[50] J. S. Horner and C. R. Contaldi, Non-Gaussian signatures of general inflationary trajectories, JCAP 1409 (2014) 001, [1311.3224].

[51] J. S. Horner and C. R. Contaldi, The bispectrum of single-field inflationary trajectories with $c_{s} \neq 1,1503.08103$.

[52] J. Elliston, D. J. Mulryne, D. Seery and R. Tavakol, Evolution of $f_{\mathrm{NL}}$ to the adiabatic limit, JCAP 1111 (2011) 005, [1106.2153].

[53] M. Dias, R. H. Ribeiro and D. Seery, The $\delta N$ formula is the dynamical renormalization group, JCAP 1310 (2013) 062, [1210.7800].

[54] M. Sasaki and E. D. Stewart, A General analytic formula for the spectral index of the density perturbations produced during inflation, Prog.Theor.Phys. 95 (1996) 71-78, [astro-ph/9507001].

[55] J. García-Bellido and D. Wands, Metric perturbations in two field inflation, Phys.Rev. D53 (1996) 5437-5445, [astro-ph/9511029].

[56] F. Vernizzi and D. Wands, Non-gaussianities in two-field inflation, JCAP 0605 (2006) 019, [astro-ph/0603799].

[57] S. Yokoyama, T. Suyama and T. Tanaka, Primordial Non-Gaussianity in Multi-Scalar Slow-Roll Inflation, JCAP 0707 (2007) 013, [0705.3178].

[58] S. Yokoyama, T. Suyama and T. Tanaka, Primordial Non-Gaussianity in Multi-Scalar Inflation, Phys.Rev. D77 (2008) 083511, [0711.2920].

[59] D. J. Mulryne, D. Seery and D. Wesley, Moment transport equations for non-Gaussianity, JCAP 1001 (2010) 024, [0909.2256].

[60] D. J. Mulryne, D. Seery and D. Wesley, Moment transport equations for the primordial curvature perturbation, JCAP 1104 (2011) 030, [1008.3159].

[61] D. Seery and J. E. Lidsey, Primordial non-Gaussianities from multiple-field inflation, JCAP 0509 (2005) 011, [astro-ph/0506056].

[62] X. Chen and Y. Wang, Large non-Gaussianities with Intermediate Shapes from Quasi-Single Field Inflation, Phys. Rev. D81 (2010) 063511, [0909.0496].

[63] X. Chen and Y. Wang, Quasi-Single Field Inflation and Non-Gaussianities, JCAP 1004 (2010) 027, [0911.3380].

[64] X. Chen and Y. Wang, Quasi-Single Field Inflation with Large Mass, JCAP 1209 (2012) 021, [1205.0160].

[65] A. Achúcarro, J.-O. Gong, S. Hardeman, G. A. Palma and S. P. Patil, Features of heavy physics in the CMB power spectrum, JCAP 1101 (2011) 030, [1010.3693].

[66] A. Achúcarro, J.-O. Gong, S. Hardeman, G. A. Palma and S. P. Patil, Effective theories of single field inflation when heavy fields matter, JHEP 05 (2012) 066, [1201.6342].

[67] A. Achúcarro, V. Atal, S. Cespedes, J.-O. Gong, G. A. Palma and S. P. Patil, Heavy fields, reduced speeds of sound and decoupling during inflation, Phys. Rev. D86 (2012) 121301, 
[1205.0710].

[68] A. Achúcarro, V. Atal, P. Ortiz and J. Torrado, Localized correlated features in the CMB power spectrum and primordial bispectrum from a transient reduction in the speed of sound, Phys. Rev. D89 (2014) 103006, [1311.2552].

[69] A. Achúcarro, V. Atal, B. Hu, P. Ortiz and J. Torrado, Inflation with moderately sharp features in the speed of sound: Generalized slow roll and in-in formalism for power spectrum and bispectrum, Phys. Rev. D90 (2014) 023511, [1404.7522].

[70] X. Gao, D. Langlois and S. Mizuno, Influence of heavy modes on perturbations in multiple field inflation, JCAP 1210 (2012) 040, [1205.5275].

[71] X. Gao, D. Langlois and S. Mizuno, Oscillatory features in the curvature power spectrum after a sudden turn of the inflationary trajectory, JCAP 1310 (2013) 023, [1306.5680].

[72] Z. Kenton and D. J. Mulryne, The squeezed limit of the bispectrum in multi-field inflation, JCAP 1510 (2015) 018, [1507.08629].

[73] Z. Kenton and D. J. Mulryne, The Separate Universe Approach to Soft Limits, 1605.03435.

[74] C. T. Byrnes, D. Regan, D. Seery and E. R. M. Tarrant, The hemispherical asymmetry from a scale-dependent inflationary bispectrum, JCAP 1606 (2016) 025, [1511.03129].

[75] T. Appelquist and J. Carazzone, Infrared Singularities and Massive Fields, Phys. Rev. D11 (1975) 2856.

[76] A. J. Tolley and M. Wyman, The Gelaton Scenario: Equilateral non-Gaussianity from multi-field dynamics, Phys. Rev. D81 (2010) 043502, [0910.1853].

[77] A. Achucarro, J.-O. Gong, S. Hardeman, G. A. Palma and S. P. Patil, Mass hierarchies and non-decoupling in multi-scalar field dynamics, Phys. Rev. D84 (2011) 043502, [1005.3848].

[78] D. Baumann and D. Green, Equilateral Non-Gaussianity and New Physics on the Horizon, JCAP 1109 (2011) 014, [1102.5343].

[79] M. Konieczka, R. H. Ribeiro and K. Turzynski, The effects of a fast-turning trajectory in multiple-field inflation, JCAP 1407 (2014) 030, [1401.6163].

[80] R. Flauger, M. Mirbabayi, L. Senatore and E. Silverstein, Productive Interactions: heavy particles and non-Gaussianity, 1606.00513.

[81] D. Seery, One-loop corrections to a scalar field during inflation, JCAP 0711 (2007) 025, [0707.3377].

[82] P. Adshead, R. Easther and E. A. Lim, The 'in-in' Formalism and Cosmological Perturbations, Phys. Rev. D80 (2009) 083521, [0904.4207].

[83] D. J. Mulryne, Transporting non-Gaussianity from sub to super-horizon scales, JCAP 1309 (2013) 010, [1302.3842].

[84] A. Berera, Warm inflation, Phys. Rev. Lett. 75 (1995) 3218-3221, [astro-ph/9509049].

[85] D. Green, B. Horn, L. Senatore and E. Silverstein, Trapped Inflation, Phys. Rev. D80 (2009) 063533, [0902.1006].

[86] M. V. Fischetti, J. B. Hartle and B. L. Hu, Quantum Effects in the Early Universe. 1. Influence of Trace Anomalies on Homogeneous, Isotropic, Classical Geometries, Phys. Rev. D20 (1979) 1757-1771.

[87] J. B. Hartle and B. L. Hu, Quantum Effects in the Early Universe. 2. Effective Action for 
Scalar Fields in Homogeneous Cosmologies with Small Anisotropy, Phys. Rev. D20 (1979) 1772-1782.

[88] J. B. Hartle and B. L. Hu, Quantum Effects In The Early Universe. 3. Dissipation Of Anisotropy By Scalar Particle Production, Phys. Rev. D21 (1980) 2756-2769.

[89] R. D. Jordan, Effective Field Equations for Expectation Values, Phys. Rev. D33 (1986) 444-454.

[90] R. D. Jordan, Expectation Values in Quantum Cosmology, Phys. Rev. D36 (1987) 3604-3613.

[91] E. Calzetta and B. L. Hu, Closed Time Path Functional Formalism in Curved Space-Time: Application to Cosmological Back Reaction Problems, Phys. Rev. D35 (1987) 495.

[92] R. P. Feynman and F. L. Vernon, Jr., The Theory of a general quantum system interacting with a linear dissipative system, Annals Phys. 24 (1963) 118-173.

[93] A. O. Caldeira and A. J. Leggett, Path integral approach to quantum Brownian motion, Physica 121A (1983) 587-616.

[94] A. O. Caldeira and A. J. Leggett, Quantum tunneling in a dissipative system, Annals Phys. 149 (1983) 374-456.

[95] P. Hajicek, Time loop formalism in quantum field theory, in Trieste 1979, Proceedings, General Relativity, Part A, 483-491, 1979.

[96] CTEQ collaboration, R. Brock et al., Handbook of perturbative QCD: Version 1.0, Rev. Mod. Phys. 67 (1995) 157-248.

[97] J. Collins, Foundations of perturbative QCD. Cambridge University Press, 2013.

[98] D. Seery, One-loop corrections to the curvature perturbation from inflation, JCAP 0802 (2008) 006, [0707.3378].

[99] L. Senatore and M. Zaldarriaga, On Loops in Inflation, JHEP 1012 (2010) 008, [0912.2734].

[100] V. Assassi, D. Baumann and D. Green, Symmetries and Loops in Inflation, JHEP 02 (2013) 151, [1210.7792].

[101] D. Seery, Infrared effects in inflationary correlation functions, Class.Quant.Grav. 27 (2010) $124005,[1005$. 1649].

[102] D. J. H. Chung, E. W. Kolb, A. Riotto and I. I. Tkachev, Probing Planckian physics: Resonant production of particles during inflation and features in the primordial power spectrum, Phys. Rev. D62 (2000) 043508, [hep-ph/9910437].

[103] O. Elgaroy, S. Hannestad and T. Haugboelle, Observational constraints on particle production during inflation, JCAP 0309 (2003) 008, [astro-ph/0306229].

[104] A. E. Romano and M. Sasaki, Effects of particle production during inflation, Phys. Rev. D78 (2008) 103522, [0809.5142].

[105] C. Burrage, R. H. Ribeiro and D. Seery, Large slow-roll corrections to the bispectrum of noncanonical inflation, JCAP 1107 (2011) 032, [1103.4126].

[106] M. Dias, J. Elliston, J. Frazer, D. Mulryne and D. Seery, The curvature perturbation at second order, JCAP 1502 (2015) 040, [1410.3491].

[107] J. R. Fergusson and E. P. S. Shellard, The shape of primordial non-Gaussianity and the CMB bispectrum, Phys. Rev. D80 (2009) 043510, [0812.3413]. 
[108] J. R. Fergusson and E. P. S. Shellard, Primordial non-Gaussianity and the CMB bispectrum, Phys. Rev. D76 (2007) 083523, [astro-ph/0612713].

[109] J. Elliston, L. Alabidi, I. Huston, D. Mulryne and R. Tavakol, Large trispectrum in two-field slow-roll inflation, JCAP 1209 (2012) 001, [1203.6844].

[110] S. A. Kim, A. R. Liddle and D. Seery, Non-gaussianity in axion Nflation models, Phys.Rev.Lett. 105 (2010) 181302, [1005.4410].

[111] S. A. Kim, A. R. Liddle and D. Seery, Non-gaussianity in axion N-flation models: detailed predictions and mass spectra, Phys.Rev. D85 (2012) 023532, [1108.2944].

[112] V. Assassi, D. Baumann, D. Green and L. McAllister, Planck-Suppressed Operators, JCAP 1401 (2014) 033, [1304.5226].

[113] G. I. Rigopoulos, E. P. S. Shellard and B. J. W. van Tent, Non-linear perturbations in multiple-field inflation, Phys.Rev. D73 (2006) 083521, [astro-ph/0504508].

[114] G. Rigopoulos, E. Shellard and B. van Tent, Quantitative bispectra from multifield inflation, Phys.Rev. D76 (2007) 083512, [astro-ph/0511041].

[115] J. Frazer, Predictions in multifield models of inflation, JCAP 1401 (2014) 028, [1303.3611].

[116] C. M. Peterson and M. Tegmark, Testing Two-Field Inflation, Phys.Rev. D83 (2011) 023522, [1005.4056].

[117] M. Alvarez et al., Testing Inflation with Large Scale Structure: Connecting Hopes with Reality, 1412.4671. 